\title{
Control of reactions and network structures of epoxy thermosets
}

\author{
Thomas Vidil $^{\mathrm{a}}$, François Tournilhac ${ }^{\mathrm{a} *}$, Simone Musso ${ }^{\mathrm{b}}$, Agathe Robisson ${ }^{\mathrm{b}}$, Ludwik \\ Leibler $^{a}$ \\ ${ }^{a}$ Ecole Supérieure de Physique et de Chimie Industrielles, 10 rue Vauquelin, 75005, Paris, \\ France. \\ b Schlumberger-Doll Research Laboratory, 1 Hampshire street, Cambridge, Massachusetts \\ 02139, United States.
}

\begin{abstract}
Recent advances in macromolecular chemistry have revolutionized the way we perceive the synthesis of polymers. Polymerization, to be modern, must be "controlled", which usually means capable of producing macromolecules of well-defined structure. The purpose of this review is to examine how the chemistry of epoxy resins, an almost century-old chemistry, is also involved in this movement.

Epoxy resins are characterized by both the flexibility of implementation and the qualities of the polymers obtained. Key materials in health-, mobility- and energy related technologies, these resins are heavily present in high-performance composites, electronic boards, adhesives and coatings. Currently, a large number of resins and hardeners are available on the market or described in the literature and an interesting point is that almost any combination of the two is possible. Common to all these recipes and processes is that a liquid (or soluble) resin at some point becomes insoluble and solid. It is very important to know how to manage this transition, physically known as the gel point, as it is the point after which the shape of the object is irreversibly set. Taking into account the variety of epoxy polymerization processes polyaddition, anionic or cationic polymerization - we detail a number of methods to program the occurrence of the gel point and how this type of control affects the structure of the growing network.
\end{abstract}

Keywords: Epoxy resins, gelation, latent curing agents, thermo- and photo-generated initiators, transfer agents.

* Corresponding author: françois.tournilhac@espci.fr, Tel: +33 140794630

Article publié dans Progress in Polymer Science 2016, 62, 126-179 


\section{Contents}

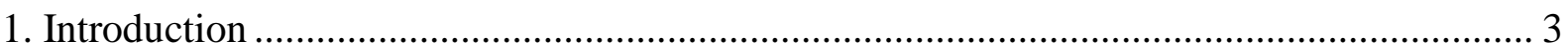

2. Epoxy thermosets: From monomers to crosslinked materials ............................................ 4

2.1. Typical epoxy resins and monomers and their polymer growth mechanisms........ 4

2.1.1. Typical epoxy resins and monomers ..................................................... 4

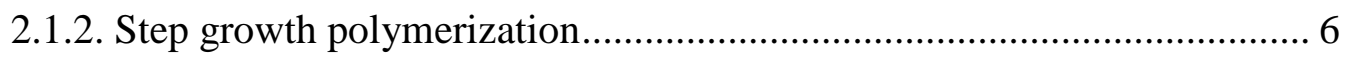

2.1.3. Chain growth ring-opening polymerization........................................ 7

2.2. Physical transitions during crosslinking and industrial concerns ......................... 11

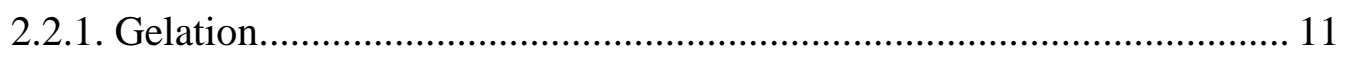

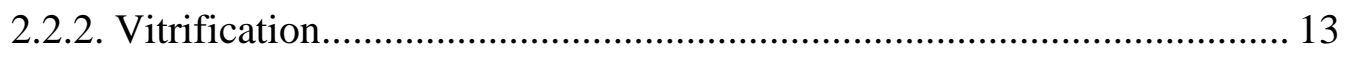

2.2.3. Industrial processes and their demands ............................................. 14

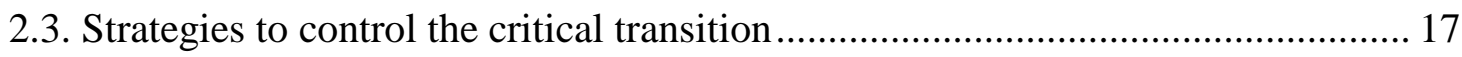

2.3.1. Cure schedule and curing temperature .............................................. 17

2.3.2. Control of the polymerization onset ................................................. 18

2.3.3. Control of the chain growth process .................................................. 21

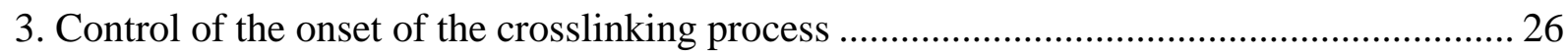

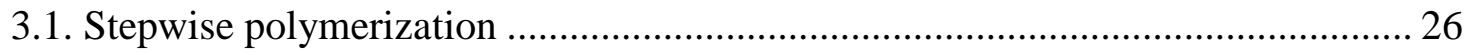

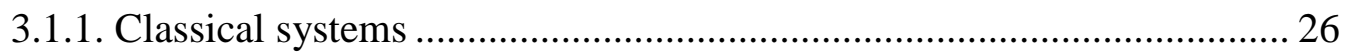

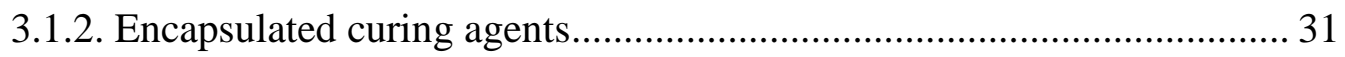

3.1.3. End capped curing agents ...................................................................... 34

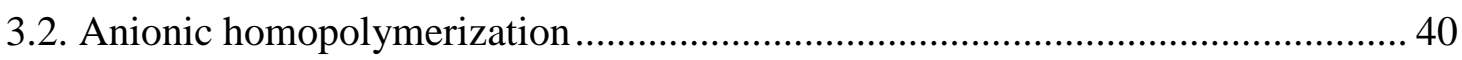

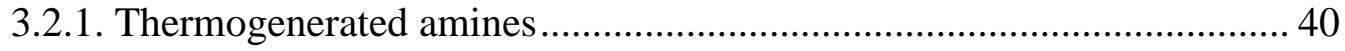

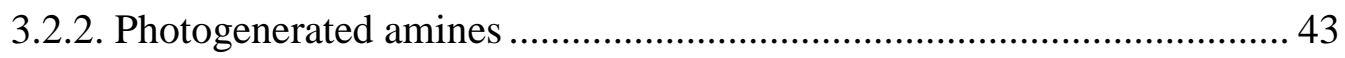

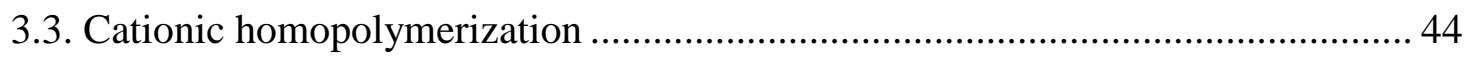

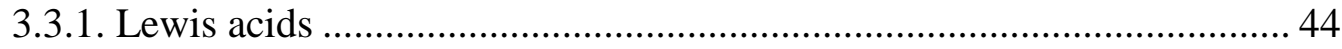

3.3.2. Onium salts ............................................................................... 49

3.3.3. Supramolecular control of the initiation ...............................................5 58

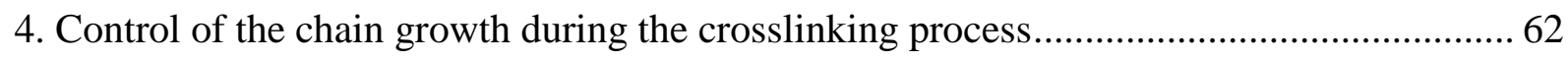

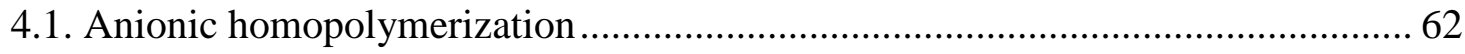

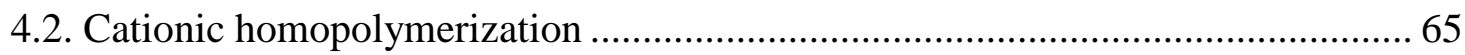

4.2.1. Chain transfer and activated monomer (AM) mechanism..................... 65

4.2.2. Control of the critical conversion .......................................................... 67

4.2.3. Control of the polymerization rate.................................................... 71

4.2.4. Combination of transfers and supramolecular complexation ................ 76

4.2.5. Toward clock like curing systems ................................................ 78

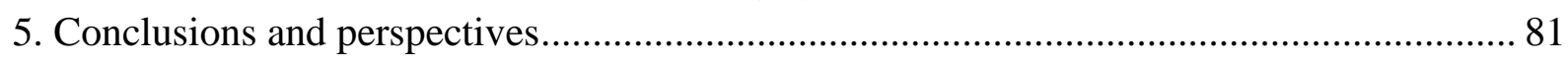

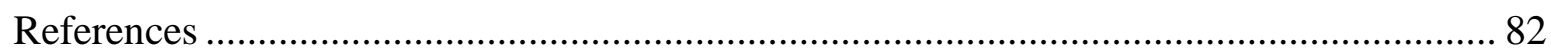




\section{Introduction}

In 1907 Leo Baekeland invented the first synthetic polymer, a phenol-formaldehyde resin called Bakelite that is made of chemically crosslinked polymer chains [1]. As such, it was the first specimen of a whole class of polymer materials: the so-called thermoset polymers [2]. Since then, polymer chemistry has experienced an outstanding rise from both the scientific and economic viewpoint. The global polymer market is measured in hundreds of millions of metric tons where thermoset polymers account for about $10 \%$ of the total production. They are largely outweighed by thermoplastic polymers [3], i.e. long polymer chains associated through intermolecular connections such as entanglements, hydrogen bonds and glassy domains [4]. However, they are still irreplaceable due to their superior mechanical properties and chemical resistance imparted by chemical crosslinks.

From the processing viewpoint, thermosets are generally made of an initial liquid mixture of polyfunctional organic molecules that gradually react with each other to form a three dimensional solid network while thermoplastics are commercialized in the form of solid pellets that are melted and shaped into their final form. Advantageously, the low molar weight of thermoset precursors permits to formulate low-viscosity reactive mixtures that offer unique flexibility in application such as coating or wetting of fibers. However, it also requires a fine control of the viscosification kinetic that eventually comes with a transition from the liquid to the solid state: the gelation $[5,6]$. This is true, for obvious reasons, in the most common engineering processes including injection and molding [7-10]. It is also the case when solid fillers (e.g. in composites [11]) or a gaseous phase (e.g. in foams [12]) are blended in the system prior to its solidification. In this case, the viscosification profile must be compatible with a homogeneous and stable dispersion of the second phase. Moreover, the control of the liquid-solid transition is still an ongoing topic of research in the most recent technologies using thermoset materials such as self healing polymers based on vascular approaches [13] or 3D printing [14].

Among the numerous chemistries used in thermoset technologies, oxirane (also called epoxy) based thermosets $[15,16]$ hold a prominent position which is intimately related to the molecular structure of the monomers. Indeed, in the three membered rings' family, a ring strain energy of about $115 \mathrm{~kJ} / \mathrm{mol}$ is estimated [17], mostly due to bond angle deformation. Thus small heterocyclic rings like oxirane but also aziridine [18] or thiirane [19] display rather strong reactivities dominated by the effects of ring strain, which promotes all types of 
ring openings. Ring opening reactions are not only versatile and self-powered processes but also permit bond formation with limited shrinkage and no release of small molecule. However, this high reactivity has also disadvantages: aziridine and thiirane appear as rather unusual and deleterious compounds [20, 21]. In this context, epoxies represent a notable exception as they are widely present in organic synthetic chemistry, in engineering and even in hardware stores, in the popular form of epoxy glues. The reason of this success is the existence of easy to manipulate, non-volatile epoxy compounds that in fact, retain all aforementioned qualities of small rings molecules without having the drawbacks. Thus, they can yield crosslinked networks either by step growth polymerization (with amine for instance) or by chain growth ring opening polymerization (in the cationic or anionic mode). The richness of this chemistry offers the possibility to control the polymerization process through its different stages: from the initiation to the propagation. Most of the industrial solutions are currently based on robust systems that polymerize only after exposure to highly energetic stimuli (high temperatures or UV irradiation). However, under the increasing demand for greener solutions, more and more systems are now designed to polymerize in conditions close to the ambient (low temperatures or visible light).

In this article, various approaches to control the onset of polymerization and the chain growth process of epoxy thermosets are reviewed. The selected examples embrace the diversity of initiation stimuli - thermal treatment and irradiation - as well as chain growing mechanisms step and chain growth processes. Special attention is paid to the latest developments reported in the literature and to the systems that offer more sustainable and energy saving solutions.

\section{Epoxy thermosets: From monomers to crosslinked materials}

\subsection{Typical epoxy resins and monomers and their polymer growth mechanisms}

\subsubsection{Typical epoxy resins and monomers}

While the term "epoxy" can be used to describe a large variety of molecules containing an oxirane group, "epoxy resins" describes a broad class of molecular structures that contain at least two oxirane groups [15, 16, 22] (Figure 1A and Figure 1B). They are often called "prepolymers" in reference to their use as building block of thermosetting polymers, the materials obtained from their crosslinking reaction [2]. The molecular base to which the oxirane groups are attached can vary widely to yield different classes of thermosetting 
polymers. It is worth noting that the cured materials are commonly called "epoxy resins" too, even if they no longer contain epoxy groups [22].

The two first examples of epoxy resin used as precursor of thermosetting polymers were reported almost simultaneously in Europe by Pierre Castan [23] and in the United-States by Sylvan Greenlee [24]. They both described the synthesis of the diglycidyl ether of bisphenol A (DGEBA, Figure 1A) from the reaction between bisphenol A (BPA) and epichlorhydrine in the presence of sodium hydroxide [25]. This process provides a prepolymer whose $n$ value is controlled by the stoichiometry of the reactants. Today, this is still the main route used for the preparation of most of the marketed resins. Following the commercial success of BPA-based epoxy resins, many other type of resins have been synthesized and are now commercially available [26].

Among other typical epoxy resins are molecules derived from the reaction of epichlorohydrin with an aromatic amine. For instance, tetraglycidylmethylenedianiline (TGMDA, Figure 1A) is a classical resin [27] extensively used in aerospace composites.
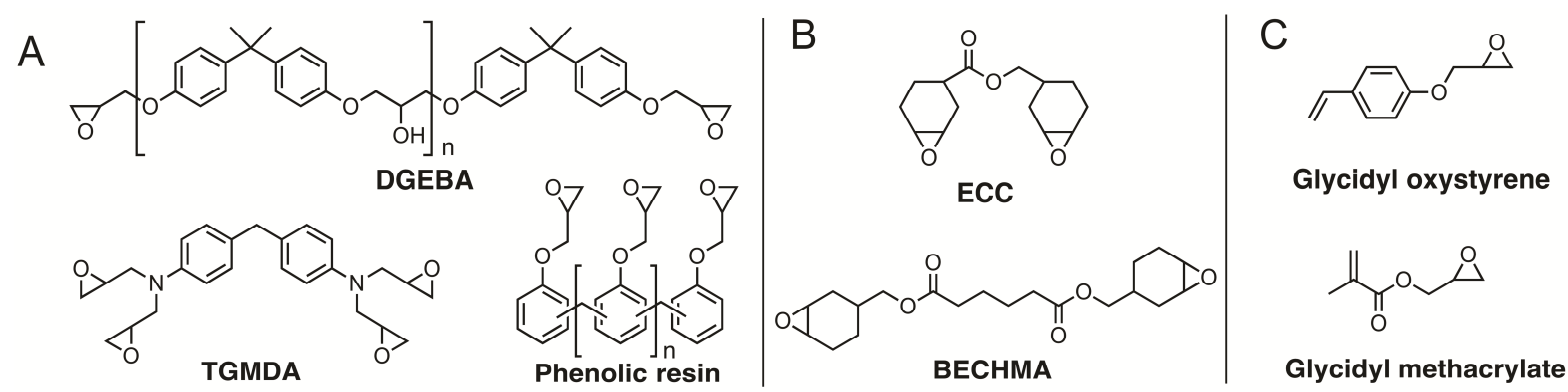

Figure 1: Typical epoxy monomers used in thermoset chemistry.

Polyglycidyl derivatives of phenolic prepolymers (phenolic resin, Figure 1A) are also common epoxy resins, known to give material with high $T_{\mathrm{g}}$ and high resistance to thermal degradation [28-30].

Cycloaliphatic resins are another class of epoxy resins of great interest [31]. They have better weather resistance and less tendency to yellow than do aromatic resins [15, 32]. Their low viscosity combined to their good electrical loss properties contributed to their commercial success in electrical and electronic applications [33, 34]. As shown in Figure 1B, with the examples of 3,4-epoxycyclohexylmethyl 3,4-epoxycyclohexanecarboxylate (ECC) and bis[3,4-epoxycyclohexylmethyl] adipate (BECHMA), they usually contain ester linkages. Due to their aliphatic nature, they are less reactive toward classical nucleophilic crosslinkers than aromatic resins and might be used to offer longer pot life [31, 35]. It is worth noting that 
the ester linkage may also react with the nucleophilic crosslinker as their use require high curing temperature [35].

In addition to these polyfunctional prepolymers, a number of monoepoxides are also used in epoxy resin technology. They are usually employed as reactive diluents that permit to reduce the viscosity of the formulation and that are incorporated in the final crosslinked network [36, 37]. Their use generally results in a faster curing rate as well as a lower crosslink density as compared to the undiluted resin [38]. Epoxy monomers containing vinyl groups are also of practical use for the synthesis of functional polymers [39]. They include glycidyl methacrylate or 4-glycidyl oxystyrene as represented in Figure 1C.

This list of resins and monomers is not exhaustive and the above-mentioned examples are just there to give an overview of the richness of the epoxy chemistry. A comprehensive list of products used in the manufacture of epoxy resins has been described elsewhere [26].

\subsubsection{Step growth polymerization}

A variety of coreactants can be used to cure epoxy resins through step polymerization processes. They include amines, acids, isocyanates and mercaptans [40].

Among them, polyamines are the most commonly used [41-43]. They involve a ring opening addition reaction. It is noteworthy that primary amine groups can react twice as shown in Figure 2.

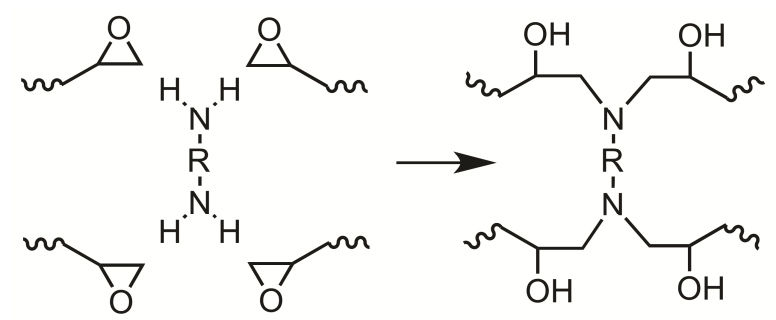

Figure 2: Reactions between a primary diamine and four epoxy moieties.

In this system the reactivity and thus, the kinetics of the crosslinking step, are dictated by the nucleophilic character of the amine and the electrophilicity of the epoxy monomer. In most cases, the epoxy resin is a derivative of DGEBA. In these conditions, the reactivity is mainly dependent upon the nucleophilicity of the amine. 
Various solutions may be envisioned to control the reactivity of the initial system. They include very simple approaches, like using a weakly nucleophilic amine [44, 45], and more sophisticated solutions, such as preventing the reaction of the amine with a protective group $[46,47]$. This will be detailed later in this review. From a more general point of view, it may be remarked that the reactivity of the $\mathrm{NH}$ functions is not constant throughout the curing process. Secondary NH functions are less reactive than primary NH groups. Moreover, as the reaction proceeds, the ring opening of the epoxy functions induces the formation of hydroxyl groups that catalyze the reaction [48]. As depicted in Figure 3, this happens through the formation of a trimolecular complex that is able to promote the nucleophilic attack of the amino group. Therefore, it is an autocatalytic reaction.

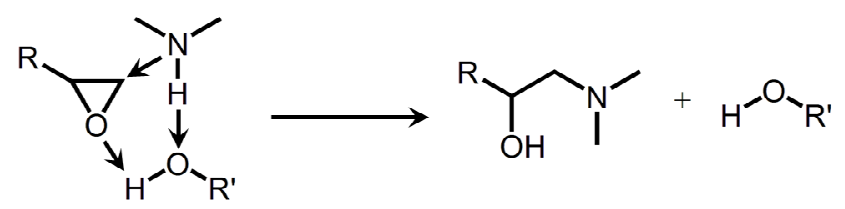

Figure 3: The catalysis of the nucleophilic attack of an amino group onto an epoxy group in the presence of an alcohol.

In this polymerization mode, the functionality of each epoxy function is one. Typically, diepoxides are used. If one wants to obtain a crosslinked network, then the coreactant monomer must present a global functionality higher than two. This is indeed the case with diamine whenever primary amine functions are present (each aminoproton reacts with an epoxy, Figure 4).

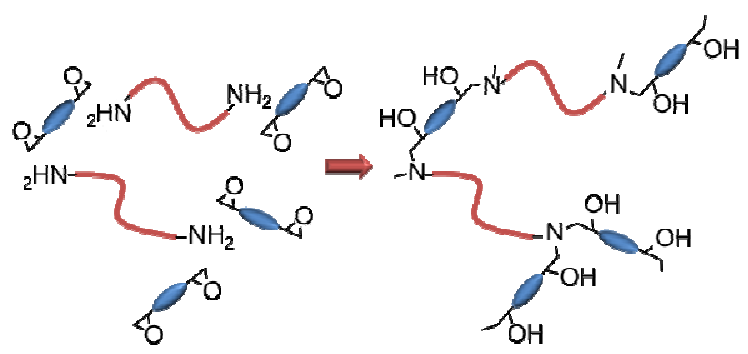

Figure 4: Growing of a three dimensional network from the reaction of a diepoxide with a diamine.

\subsubsection{Chain growth ring opening polymerization}

As a result of their three-membered ring structure, epoxies exhibit highly polar oxygencarbon bonds which are expected to break ionically [22, 49, 50]. For example, the dipole moment of ethylene oxide in benzene solution is $1.82 \mathrm{D}$ where it is only $1.1 \mathrm{D}$ for regular 
ethers [22]. Therefore, epoxies can easily be involved in either electrophilic attack through their oxygen atom or nucleophilic attack on their ring carbon atoms. In other word, they can undergo both anionic and cationic polymerization. In every case, the chain polymerization process presents the three classical distinctive stages of chain polymerization:

- The initiation during which an initiator provides a suitable active center by reacting with a monomer

- The propagation during which a series of consecutive addition of monomers to the active center is proceeding. The active center is regenerated at every step, allowing the reaction to continue.

- Eventually, the termination during which the active center is neutralized.

In view of the control of the crosslinking step, chain ring opening polymerization appears as a valuable option. Indeed, it is possible to control the kinetics through both the initiation step and the propagation. By tuning the reactivity of the initiator, one can control the occurrence of polymerization and thus of the whole crosslinking process. Once polymerization is underway, it is also possible to play with the rate of propagation for instance by adding a transfer agent. Below are presented the distinctive features of the chain ring opening polymerization in the anionic and cationic modes.

\subsubsection{Anionic polymerization}

Anionic polymerization is commonly initiated by tertiary amines and particularly imidazoles [51]. It involves the formation of an alkoxide, which then attacks a monomer while regenerating another alkoxide and so on. The mechanism is illustrated in Figure 5.
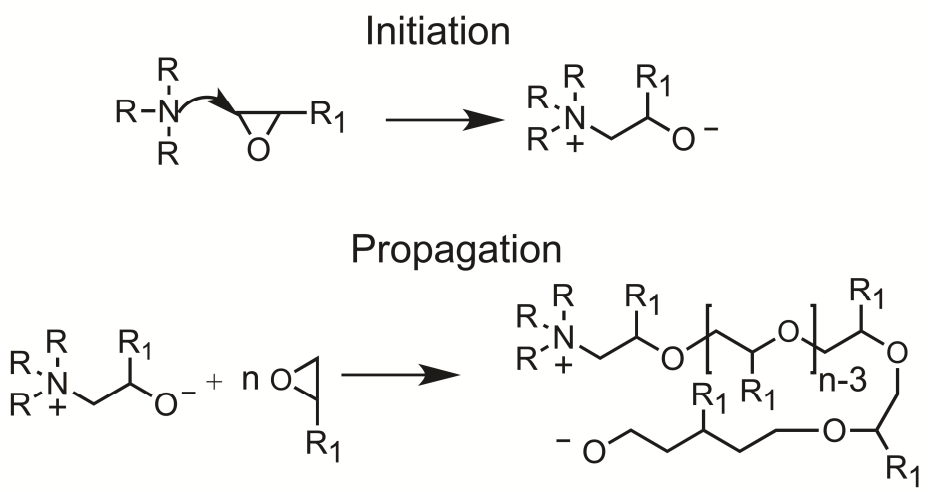

Figure 5: Anionic polymerization of epoxide initiated by a tertiary amine in the presence of an alcohol 

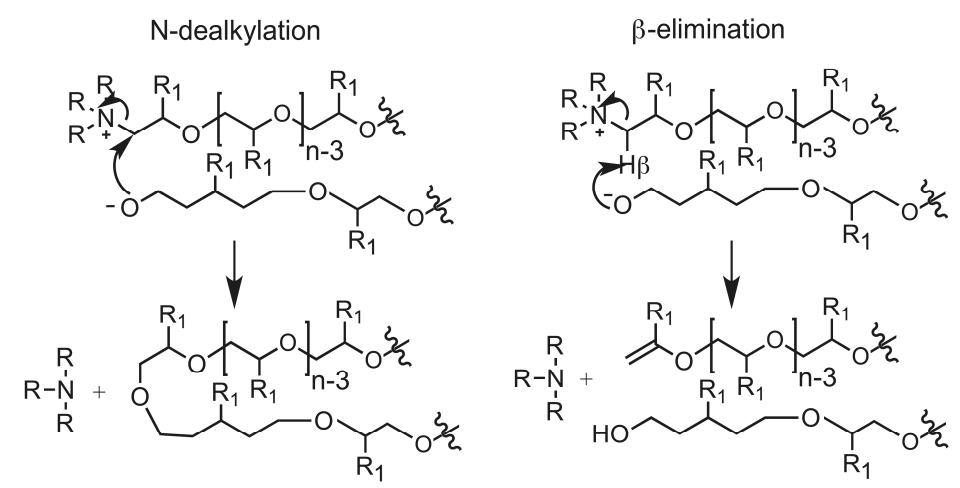

Figure 6: Possible terminations for the anionic polymerization of epoxide initiated by tertiary amines

Termination may result from $\mathrm{N}$-dealkylation or the $\beta$-elimination of the $\mathrm{N}$ substituent from the growing chain [52]. The two mechanisms are illustrated in Figure 6. They result in the regeneration of the tertiary amine.

It is worth noting that epoxies are also copolymerized with other cyclic monomers according to anionic chain polymerization processes. In particular, the curing of epoxy-anhydride formulations, using tertiary amines as initiators, has been described as an alternating epoxyanhydride anionic copolymerization [53-56]. This system has been extensively studied as anhydrides are, with primary amines, the most commonly used crosslinkers for epoxy resins [40]. Recent studies have shown that the mechanism is far more complex than a strictly alternating epoxy-anhydrides copolymerization scheme [56]. But the overall curing mechanism is commonly described as a classical anionic ring opening chain polymerization with an initiation, a propagation and a termination as described in Figure 7. Other cyclic comonomers can be copolymerized with epoxies according to an anionic alternating copolymerization mechanism and are used in epoxy thermoset technology. They include bicyclic and spirocyclic bis( $\gamma$-lactone)s [57-62]. These are comonomers dedicated to the development of shrinkage-free or expanding curing formulations. Indeed, they react according to a double ring opening mechanism that results in a molecular expansion [57]. However, in the present review, for the sake of conciseness, the discussion on anionic polymerization of epoxies will be focused on the case of homopolymerization. 
Initiation

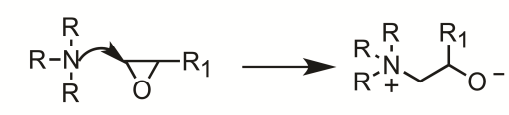

Propagation

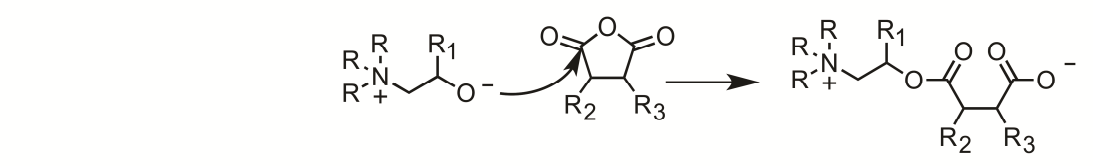

$\longrightarrow$

Termination (regeneration of the tertiary amine)

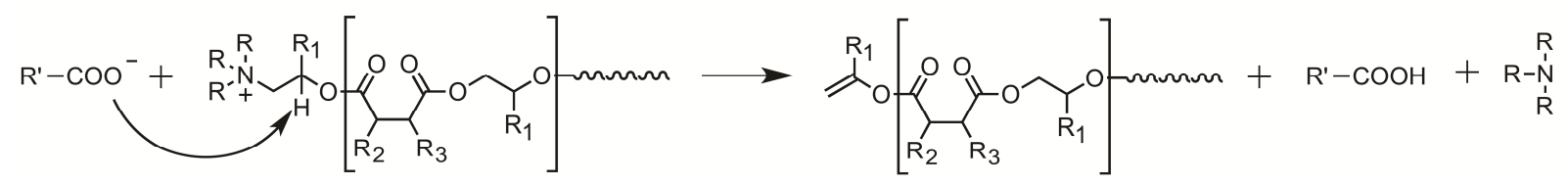

Figure 7 : The mechanism of the alternating ring-opening copolymerization of epoxides and anhydrides initiated by a tertiary amine.

\subsubsection{Cationic polymerization}

Cationic polymerization of epoxy resins is commonly initiated by boron trifluoride complexes [63] and several onium salts [64] including diaryl iodonium [65], triarylsulfonium [66] or phosphonium salts [67]. The initiation mechanisms are detailed later in the Section 3.3 of this review. Propagation proceeds through an oxonium active center at the end of the growing chain as represented in Figure 8. However, it could also proceed through an activated monomer mechanism and this will be detailed in the Section 4.2 of this review.

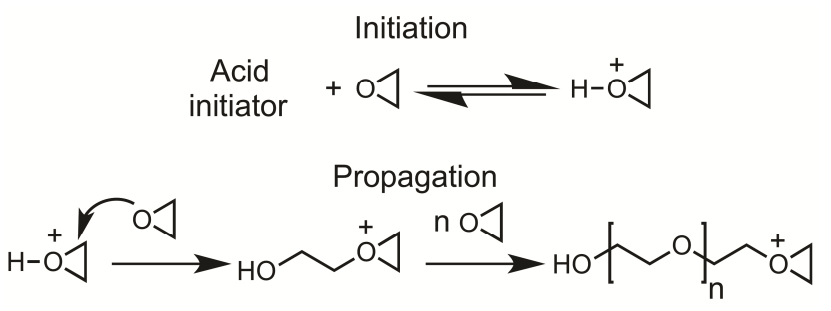

Figure 8: Cationic polymerization of epoxide

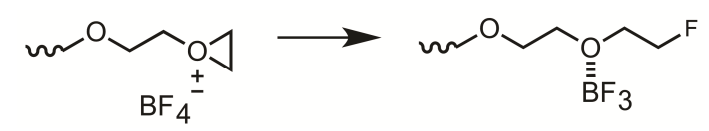

Figure 9: Possible termination for the cationic polymerization of epoxide

Combination of the propagating oxonium with an anion derived from the counteranion is thought to be one of the main termination steps. In the case of $\mathrm{BF}_{4}^{-}$, the weakly nucleophilic anion $\mathrm{F}^{-}$may attack the propagating oxonium cation as illustrated in Figure 9 [68]. 
What is important to note in the chain polymerization mode is that the functionality of each epoxy group is 2 . Therefore, as depicted in Figure 10, diepoxy monomers like DGEBA are enough to produce a polyether network through the homopolymerization of epoxide groups [69].

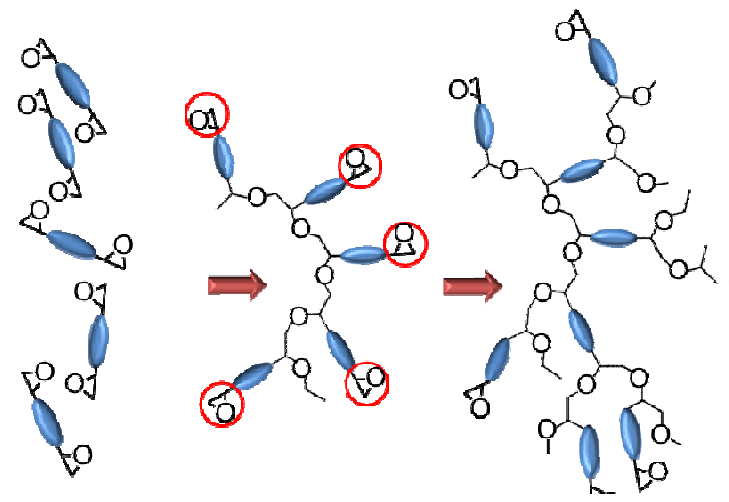

Figure 10: Growing of a three dimensional network from the homopolymerization of diepoxide molecules

In epoxy thermoset technology, cycloaliphatic resins are sometimes preferred. Because they are free of aromatic groups, they are better suited for UV initiated cationic ring opening polymerization and also less prone to coloration under UV light exposure than resin based on bisphenol A [32]. Furthermore, cationic polymerization is particularly suitable for these resins as they are very poorly reactive with common nucleophilic crosslinkers such as primary amines [31] (see Section 2.1.1.).

It is worth noting that epoxides can also be copolymerized with spirocyclic bis( $\gamma$-lactone $) \mathrm{s}$ in presence of cationic initiators [70, 71]. Similarly to what is observed in the anionic polymerization mode (see section 2.1.3.1), this approach provides curing formulation with little shrinkage or even expansion. However, in that case, the oxirane ring is not directly involved in the ring opening polymerization. Instead, it first reacts with the spirocyclic bis $(\gamma-$ lactone)s, yielding a spiroorthoester, which in turn undergoes cationic ring opening polymerization.

\subsection{Physical transitions during crosslinking and industrial concerns}

\subsubsection{Gelation}

Whatever the crosslinking process one considers, if the functionality of the monomer is adequately chosen, it is accompanied at some point by a critical transition: gelation [72]. From a pure macroscopic viewpoint, this transition, also termed the gel point, is characterized by the visible formation of a gel or insoluble polymer fraction. It involves an abrupt change 
from a liquidlike to a solidlike behavior. Figure 11 illustrates the time dependence of viscosity as a function of conversion of the epoxy groups, $x$, for the cationic curing of DGEBA [73]. When $x$ is equal to the gel conversion, $x_{g e l}$, the viscosity becomes infinite and a solid fraction is appearing whose elastic modulus increases as the conversion increases.

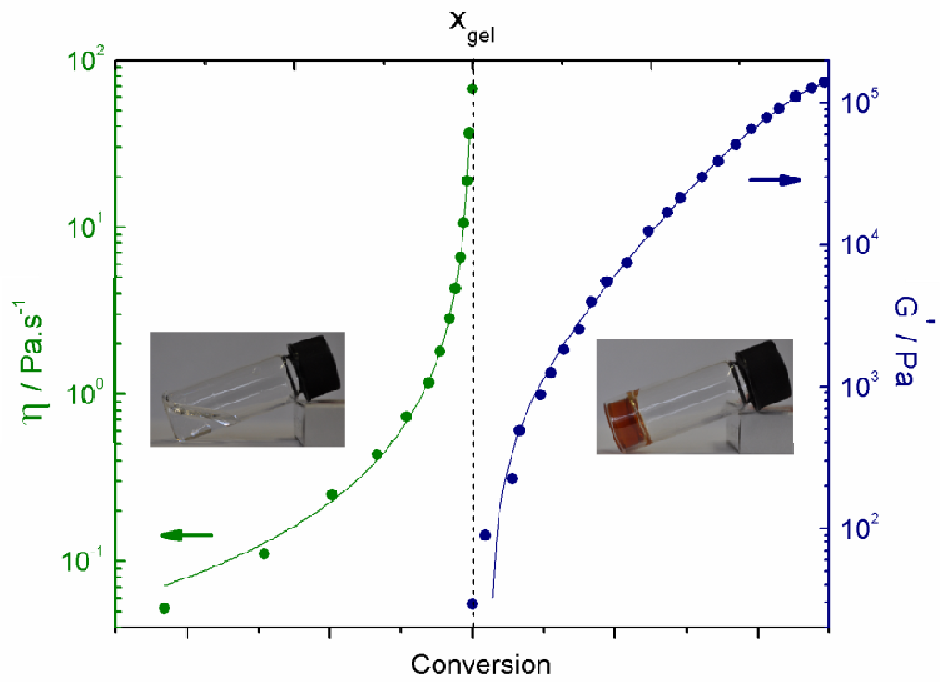

Figure 11: Evolution of the physical properties during the cationic curing of DGEBA as a function of conversion of epoxy groups

From a molecular standpoint, gelation occurs when a growing molecule reaches a mass large enough to interconnect every boundary of the system. Thus, when gelation takes place it is common to say that there is one giant molecule throughout the system. Its contribution to the total number of molecules $M_{n}$ is negligible and therefore there is no particular accident on $M_{n}$ at $x_{\text {gel }}$. However, the mass fraction of this giant molecule is significant and its contribution to the $M_{w}$ value prevails over the contribution of the much smaller species. For this reason, a mathematical definition of the gel point states that: $M_{w}$ tends to infinite when $x=x_{g e l}$.

It is interesting to note that stepwise and chainwise polymerization exhibit different behaviors in the pregel stage [74]. Step polymerization results from the reaction between reactive molecules that are homogeneously distributed in the system at the very beginning of the crosslinking process. Thus the system is cured in similar conditions in any part of the mixture as represented in the schematic illustration of Figure 12. In contrast, chain polymerization results from an initiation step starting at different discrete points of the system. The process is intrinsically inhomogeneous and may develop inhomogeneities on a nanoscale, called microgels [75]. As polymerization proceeds, microgels may be obtained in the form of crosslinked polymer coils swollen by unreacted monomers. This is illustrated in Figure 13 for the chain ring-opening polymerization of epoxy monomers and diepoxide crosslinkers. The 
dimension and concentration of the polymer coils increase until the apparition of an interconnected structure that percolates the system resulting in macrogelation $[76,77]$.

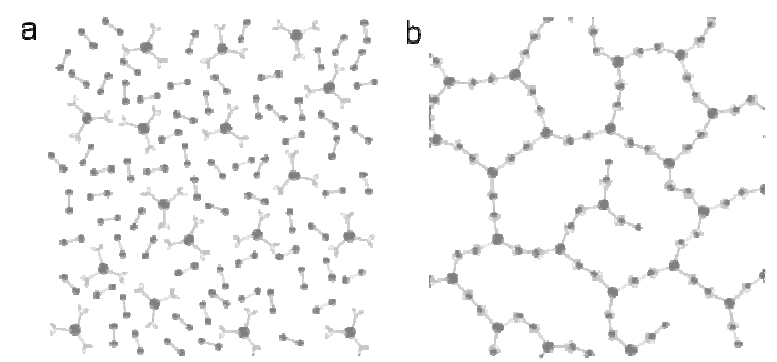

Figure 12: Schematic representation of the gelation transformation $(\mathrm{a} \rightarrow \mathrm{b})$ in step polymerization for the reaction between trifunctional and bifunctional molecules.

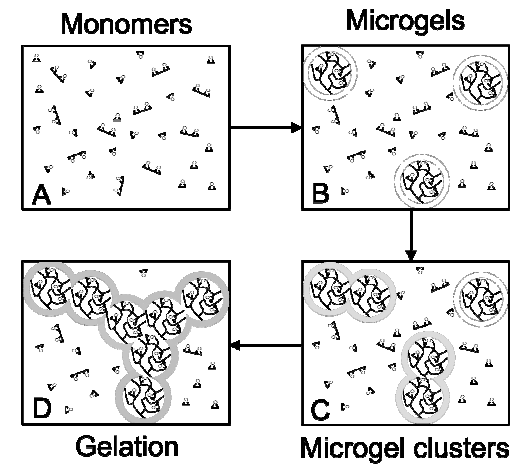

Figure 13: Schematic representation of the inhomogeneous gelation process for the chain ring-opening polymerization of epoxy monomers and diepoxide crosslinkers

From the viewpoint of kinetics and reactivity, gelation does not influence the crosslinking reaction. Indeed, the characteristic time scale related to the local mobility of functional groups after gelation is still much shorter than the characteristic time scale to produce the chemical reaction. Thus, gelation essentially affects the macroscopic behavior of the system.

\subsubsection{Vitrification}

Vitrification or glass transition is the transformation that takes place when the system is transformed from a liquid or gel into a glass. It is a consequence of the reduction of the system's mobility through the formation of covalent bonds and reduction of density, up to a point where the cooperative movement of large portions of the polymer are no longer possible. It must be emphasized that this transition may take place either before or after gelation.

Contrarily to the gelation, the definition of this transition is not independent on temperature. Indeed, the possibility of producing cooperative movements of fragments of the thermosetting polymer increases with temperature. Thus, the conversion at which glass transition appears, increases with the cure temperature. More generally speaking, independently from the degree of advancement of the curing reaction, vitrification is always a reversible process while gelation cannot be reversed in conventional crosslinked material like epoxy thermoset. 
Another significant difference with the gel point is that glass transition significantly hampers the curing process $[78,79]$. Indeed, in the glassy state, relaxation times become very long. The local mobility of functional reactive groups is considerably altered and reaction is rapidly arrested. Polymerization may restart by increasing the temperature.

\subsubsection{Industrial processes and their demands}

In the field of processing, gelation and vitrification have both significant impacts.

\subsubsection{The impact of gelation}

As it was explained in the above sections, at the gel point the system undergoes a transition from a liquid to a solid state. Thus, during processing of the epoxy formulation, gelation must be avoided prior to the shaping of the final part. A noteworthy exception is the special case of epoxy vitrimers [80-82], in which the network can be reshaped after gelation by bond exchanges.

The knowledge of the time needed to observe gelation, the gel time $t_{g e l}$, is of great practical importance in many industrial processes. For instance, several liquid composite molding techniques rely on the injection of the liquid formulations. Resin transfer molding (RTM) [8, 9,83 ] involves the pumping of the liquid precursors into a heated mold cavity containing preplaced fiber materials. Reaction injection molding (RIM) [79, 84, 85] is based on the premixture of two or more liquid components at high pressure in a hydraulically operated mixhead, followed by the injection in the mold cavity. For this system, it is important to be sure that the gel time is long enough to permit the injection in the mold prior to gelation.

Gelation is also of utmost importance in foaming processes for different reasons. Indeed, in that case, a blowing agent is added to the monomers solutions [12,86, 87]. When temperature increases as the reaction proceeds, the boiling point of the blowing agent is overstep leading to its evaporation and the subsequent formation of the foam. The end of the rise of the foam is determined by the gelation. Thus gelation determines the maximum height and the apparent density of the final foam.

It is also noteworthy that the morphology of nanostructured materials is very dependent on the occurrence of the gel point. When block copolymers are dispersed in a hard epoxy matrix, the final size of their inclusions in the network is dictated by the occurrence of gelation that 
precludes the relaxation of their morphology $[88,89]$. In that case, the mechanical and optical properties of the materials are markedly influenced by the curing kinetics.

The fundamental need to control gelation in thermoset technology is also well illustrated in the latest discoveries of material science.

As an example, in the field of self-repairing material, White et al. recently reported a strategy to restore mechanical performance in response to large-scale damages [13]. They used a vascular approach, i.e. based on the delivery of reactive fluids through two independent networks to the site of damage. This approach requires low viscosity components in order to mix and quickly wet the inner surface of the damage. In the mean time, for large damages, the viscosity must be high enough to prevent the effect of gravity that may cause the fluid to drip out of the damage region. To address this problem, White et al. proposed a two-stage restoration process where a first gel is formed with a very short gel time, $t_{g e l}(1)$, in order to form a mechanical support that retain the unreacted fraction of the fluid. The latter forms a stiffer gel after a longer period of time, $t_{g e l}(2)$, once the damage is completely filled with additional fluid coming from the microvascular channels. The two-stage restoration strategy is illustrated in Figure 14. It is an interesting example where gelation of thermosetting polymers must be precisely controlled to respect a complex cure schedule.

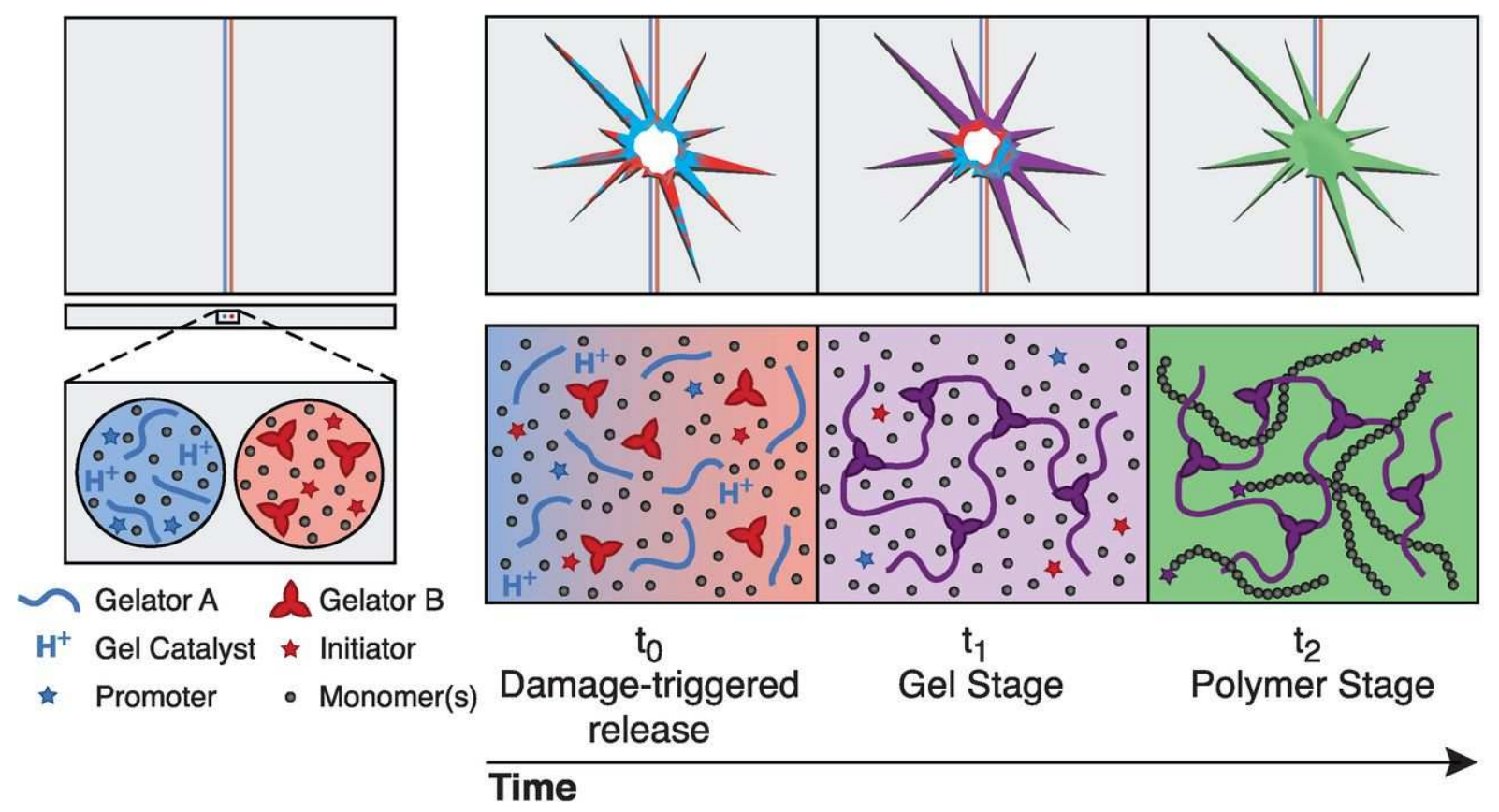

Figure 14: Schematic representation of the two-stage restoration strategy developed by White and coworkers. Reactive monomer solutions are incorporated into a vascularized sample (blue and red channels). After an impact, chemicals are released into the damage region $\left(t_{0}\right)$. A first gel (purple) is rapidly formed $\left(t_{1}\right)$. The migration of fluid and gelation continues until the void is filled. At time $t_{2}$, the gelation of the second chemical system results in recovery of structural performance (reproduced from (13) with permission of AAAS). 
Similarly, in 3D printing technologies, the control of the occurrence of the gel point is of utmost importance. Indeed, some methods using thermoset materials or hydrogels are based on the layer-by-layer deposition of a monomer solution on the printing substrates as depicted in Figure 15. In that case, gelation must be quick enough to prevent the collapse of the structure obtained after extrusion [14].

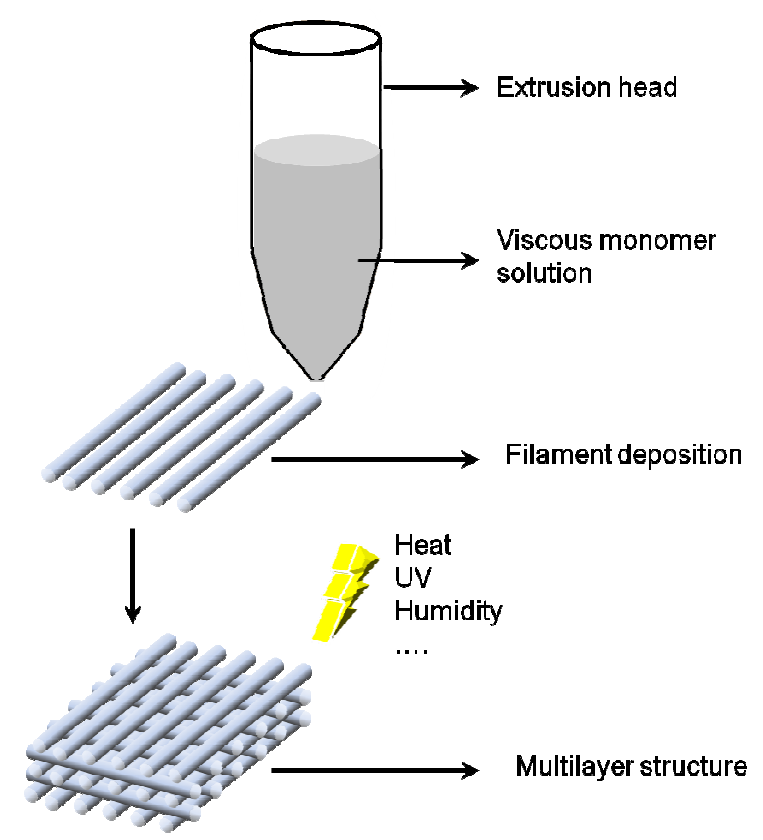

Figure 15: Printing process for the multilayer deposition of thermosetting polymers. Post-printing treatment enables the curing of the deposited system. The most widespread solution is UV exposition.

\subsubsection{The impact of vitrification}

As previously underlined, the influence of vitrification on the thermoset cure is very important because the polymerization kinetics is severely hindered as soon as the material enters the glassy region [79].

In some cases, this may be used as a valuable tool to store partially reacted epoxy formulations in the glassy state [90,91]. Again, with the exception of vitrimers, this has to be accomplished before the gel point is reached in order to produce the final shaping without internal stresses when reheating above $T_{g}$.

However, most of the time, as for gelation, the occurrence of vitrification during cure is a problem, especially for curing processes carried out at room temperature without any external heat source. This is the case for polymerizations activated by UV for instance. In this 
condition, the temperature increase resulting from the exothermic polymerization has to be sufficient to permit the completion of the crosslinking process.

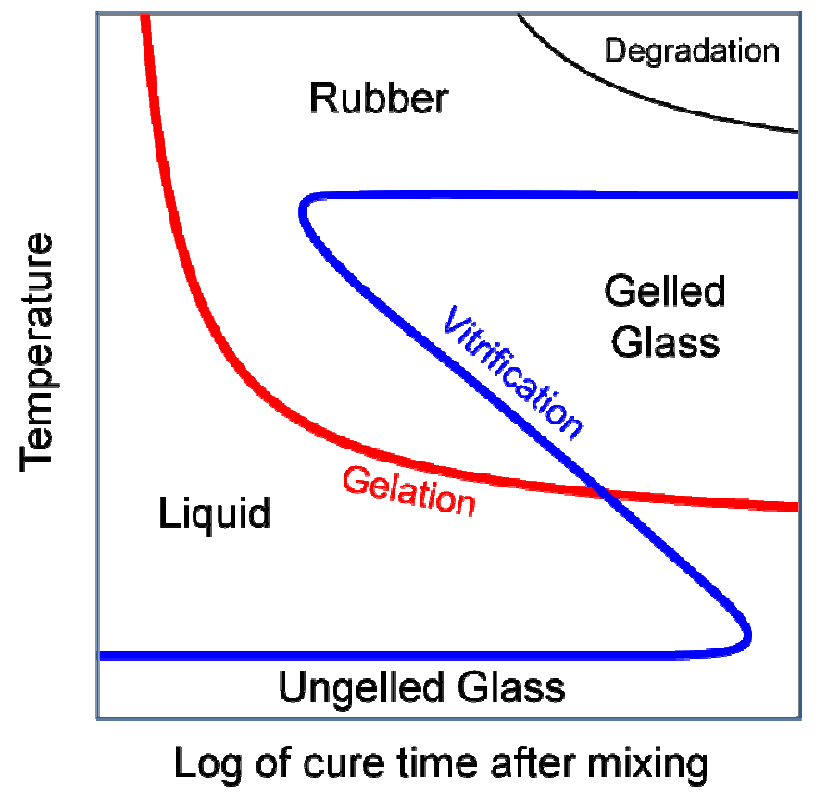

Figure 16: A typical time-temperature transformation (TTT) diagram for a thermosetting polymer.

\subsection{Strategies to control the critical transition}

\subsubsection{Cure schedule and curing temperature}

To overcome the above-mentioned problems related to the onset of gelation and vitrification, classical solutions rely on the choice of an appropriate thermal cycle. Indeed, most of common thermoset resins used in the industry are thermally activated systems. Thus, in these cases, the knowledge of the gelation and vitrification times of the curing system at any temperatures is enough to devise a convenient cure schedule for a particular application.

Gelation, vitrification but also degradation are conveniently represented in temperature $v s$ time transformation (TTT) diagrams as introduced by Gillham and coworkers [92-95]. Figure 16 shows a typical TTT diagram for a thermoset polymer. It is a dynamic phase diagram where the gelation curve (red), the vitrification curve (blue) and the degradation curve (black) set the limits of the different phases as a function of the curing time and the curing temperature. The four possible phases are the liquid (before gelation), the rubber (after gelation but above the glass transition temperature, $T_{g}$ ), the gelled glass (after gelation and above the glass transition temperature, $T_{g}$ ) and the degraded polymer. Interestingly, the ungelled glass region is the region where the reaction is physically hindered. Vitrification 
prevents any reaction and the system may be indefinitely stored under this form (see Section 2.2.3.2).

In common industrial processes, the initial temperature, $\mathrm{T}_{0}$, is chosen high enough to be placed in the liquid region of the TTT diagram. A low viscosity formulation is obtained, enabling the shaping of the part. In the mean time, $T_{0}$ is chosen sufficiently low to prevent premature curing that will result in gelation and/or vitrification. The temperature is then raised in a controlled manner to prevent excessive heat dissipation that will result in monomer degradation and cross the gelation curve where and when it is desirable.

However, whatever the temperature one considers in the liquid region, after a given period of time, the system will cross either the gelation or the vitrification curves, and shaping will be prevented. In most of the cases, after mixing of the reactants, this period of time is pretty short (hours) and it is desirable to handle it through another lever than temperature. A better control is achieved from the kinetics viewpoint with well-formulated systems. Reactants, initiators and sometimes inhibitors can be selected to program the pot life of the mixtures.

\subsubsection{Control of the polymerization onset}

\subsubsection{Latency}

In most cases, epoxy reactive mixtures are prepared from two components (for instance a multivalent epoxy and a multivalent amine, or a multivalent epoxy and an initiator) that have to be stored separately and mixed prior to use. The first solution to control the occurrence of gelation and/or vitrification consists of controlling the onset of the polymerization process. As long as the polymerization is not running, the system stays in its liquid form and can be handled for injection, coating and so on. If we refer to the above mentioned TTT diagrams, the aim here is to design a system that will indefinitely stay in the liquid region and that will cross the gelation curve where and when the operator decides. This is a more suitable option as compared to the strategy that consists to store the system in the ungelled glassy region where the resin cannot flow.

Latent curing agents have been designed for one-pot curing systems with long shelf life [96, 97]. They are made of two well-chosen components that cannot react under normal conditions. External stimulations are necessary to trigger the curing process [64]. They include heating, photoirradiation, pressure, magnetism, ultrasonic wave, electron beam, X- 
rays and so on. Heating and photoirradiation are the most appropriate for obvious reasons of cost and safety.

The major advantage of this approach is to combine both commercial and processing advantages. On one hand, the system can be stored under the form of a one-pot curing system over a very long period (in the liquid region). On the other hand, polymerization and subsequent gelation can be triggered at the exact time and place desired by the operator.

It is important to note that curing systems must verify strict criteria to receive the appellation "latent" [97]. Indeed, a true latent curing system strictly shows no activity under normal conditions. The relationship between external stimulation and the monomer conversion of different systems is illustrated in Figure 17. System C and D are so called latent curing systems because they do not exhibit any reaction in normal conditions. System $C$ is a better latent system than $\mathrm{D}$ due to a better responsiveness as the stimulation increases. A sharp increase of conversion as the stimulation is applied is an advantage from the processing viewpoint. System A presents an increase of monomer conversion under increasing stimulation. However, its activity in normal conditions is not zero. Then, A is not a true latent system.

In the field of epoxy chemistry, various latent systems have been described for both step and chain ring-opening polymerizations. They are based on the release of reactive functions after cleavage of covalent or physical bonds. They are reviewed in Section 3 of the present paper.

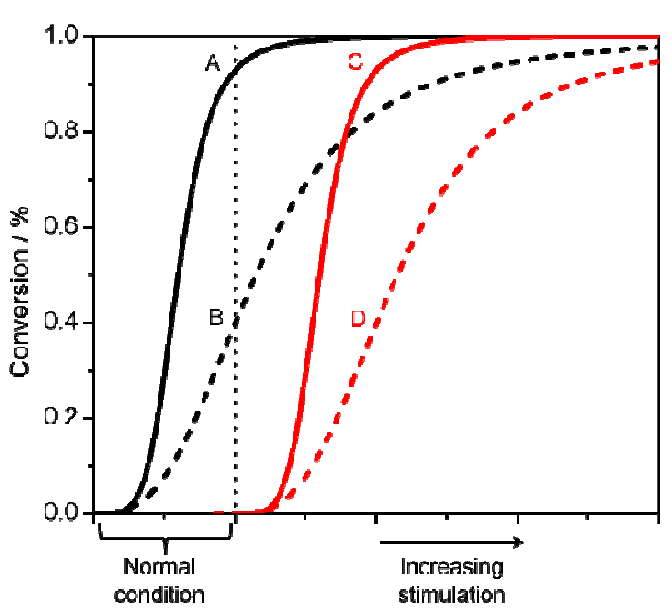

Figure 17: Relationship between external stimulation and monomer conversion for different curing systems. Plot $\mathrm{A}=$ system with no latency that reacts quickly under normal conditions, Plot $\mathrm{B}=$ system with no latency that reacts slowly under normal conditions, Plot $\mathrm{C}=$ latent system that reacts quickly under stimulation, Plot $\mathrm{D}=$ latent system that reacts slowly under stimulation

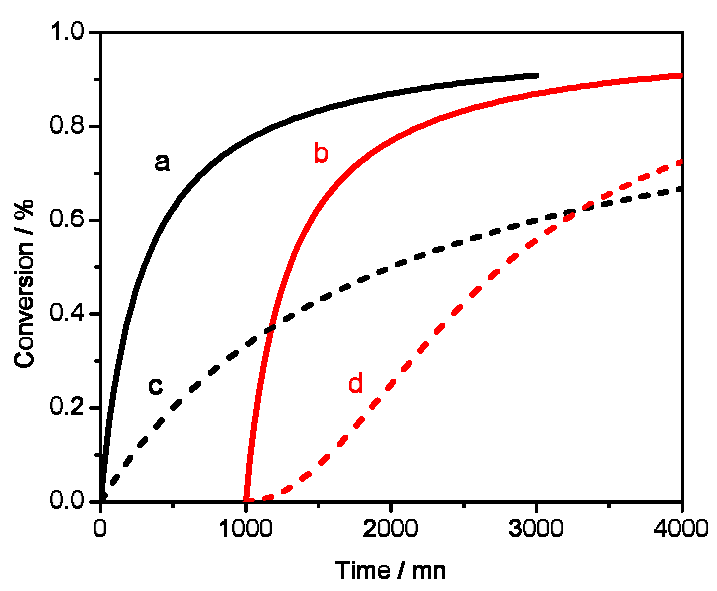

Figure 18: Typical conversion profiles obtained for inhibited or retarded polymerization. Plot a $=$ system free of retarder or additive, Plot $\mathrm{b}=$ profile obtained with an ideal inhibitor, Plot $\mathrm{c}=$ profile obtained with a retarder, Plot $\mathrm{d}=$ profile obtained with a retarder that also exhibit an induction period. 


\subsubsection{Inhibition and retardation}

Latent systems are externally stimulated multicomponent curing systems for which the epoxy formulation is blocked in the liquid region of the TTT diagram. The epoxy resin does not react with the hardener or the initiator as long as no external stimulus is applied.

For some applications, it is sufficient to lengthen the lifetime of the system in this state. This can be achieved, without playing with temperature, by using appropriate additives called inhibitors and retarders [98-100].

Ideal inhibitors are defined as substances that cause an induction period in the polymerization of monomers, during which the inhibitor is consumed and after which polymerization starts at its normal rate (Figure 18, plot b). Typical retarders are defined as substances that cause retardation during the entire polymerization, without giving rise to an induction period (Figure 18, plot c). Inhibitors practically block all active centers produced during the initiation, but retarders are less efficient and permit some polymer chains to be initiated. They react with these growing chains and terminate them, thus acting like chain-breakers and reducing the molecular weight of the polymer. In thermoset technology, this means more dandling chains and, as a result, lower crosslink density and lower $T_{\mathrm{g}}$.

Inhibitors are commonly added to curing formulations to induce an induction period in very reactive systems. Indeed, for some two components systems that undergo rapid polymerization once the mixing process is underway, it is highly desirable to induce an induction period that is long enough to thoroughly mix the components and then process the reactive mixture (shaping, coating, etc.) before the gel point and/or the vitrification. This is an approach specific to the chain polymerization mode.

It is commonly used for the free radical polymerization of vinylic monomers. For instance, Li and coworkers described the use of quinone and nitrobenzene to adjust the resin's gel time for the radical crosslinking polymerization between styrene and vinyl ester resin [101] used in applications such as RIM and RTM. As for chain polymerization of epoxy, several inhibited or retarded systems have been reported in the case of cationic polymerization. A number of nucleophilic species can compete with monomers in the attack of cationic reactive centers (i.e. protons, activated monomers, activated chain ends) as described in Figure 19. In particular, amines were reported as effective inhibitors of the cationic polymerization of epoxy resins [102-104]. Water and alcohols are also commonly reported as retarder of 
cationic polymerization $[105,106]$. They are not inhibitors as the oxonium ions they form are not stable enough to retain the active protons on a long period. Instead, they promote transfers and decrease the overall rate of the polymerization. It is worth noting that alcohols and water could also accelerate the curing rate as the role they play is very dependent on the structure of both the monomers [107, 108] and the initiator [109]. This will be further discussed in Section 4.2. On a more anecdotal level, several studies suggest that dialkyl sulfides might be efficient inhibitors of the cationic polymerization of epoxy resins $[110,111]$. They have been used to develop latent two-stage formulations that are activated by UV irradiation and subsequently polymerized by increasing the temperature. The thermoresponsive system obtained after UV irradiation results from the reaction of the dialkyl sulfides with UV-generated activated monomers (or activated chain ends) to form stable trialkylsulfonium salts.

In Section 4 are reported recent discoveries that have enabled the design of epoxy curing systems with well-controlled induction periods.

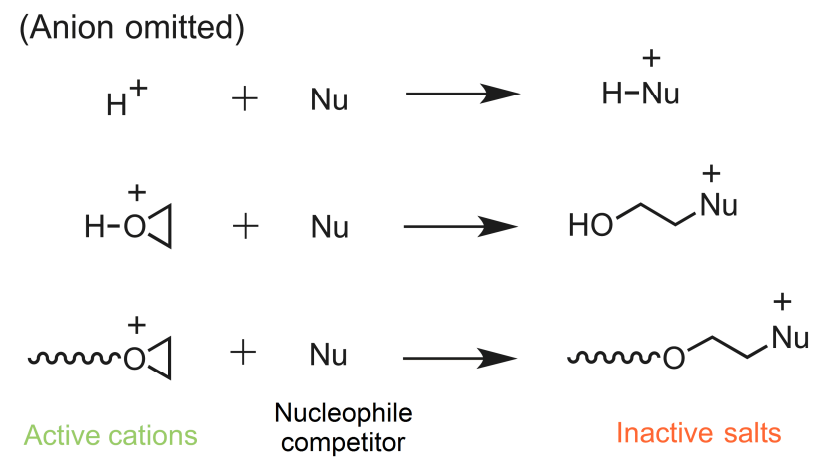

Figure 19: Different reactions that may result in the inhibition of the cationic ring-opening polymerization of epoxides in the presence of a nucleophilic competitor.

\subsubsection{Control of the chain growth process}

\subsubsection{Gel time and critical conversion}

The general strategies to control the onset of polymerization have been briefly introduced in Section 2.3.2. Latent initiating systems or inhibitors are among classical solutions. However once the polymerization is underway, there is still room to handle the curing system. As long as the gel point is not attained (i.e. the system is still in the liquid region of the TTT diagrams) and providing the viscosity is not too high, it is possible to manipulate the liquid system. Thus, controlling the time between the onset of polymerization and the gel point (the gel time) provides another lever to optimize the processing of thermoset as suggested in Figure 20. This 
could be achieved by tuning the polymerization rate with temperature or retarders (see section 2.3.2.2).

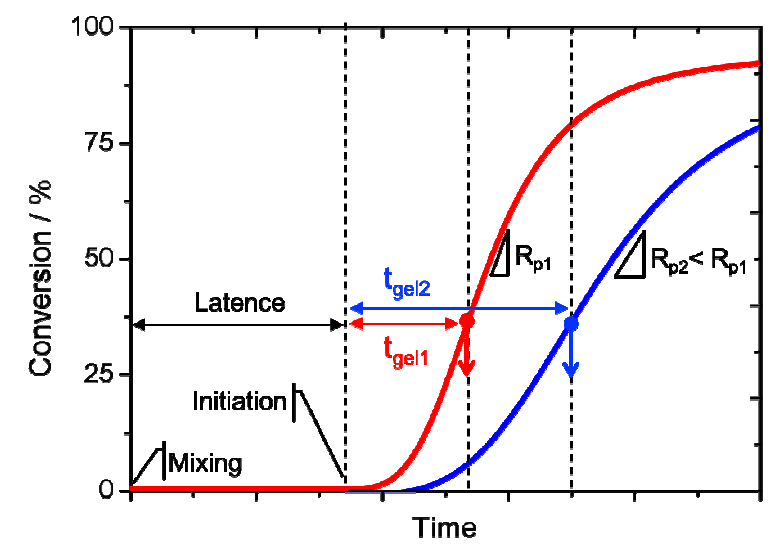

Figure 20: Control of the gel time through the polymerization rate. Typical configuration when a retarder agent is used.

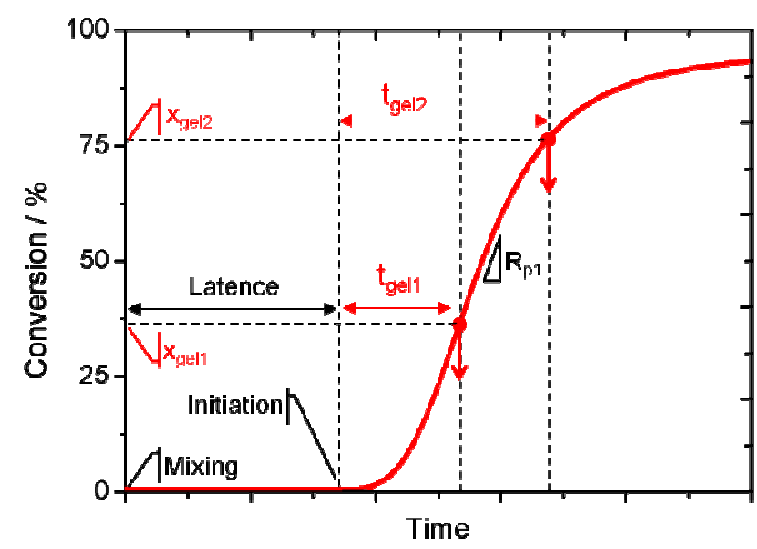

Figure 21: Control of the gel time through the critical conversion. Typical configuration when a chain transfer agent (CTA) is used.

In a similar vein, the conversion at the gel point, $x_{g e l}$, also called critical conversion, is a parameter of practical importance. From the viewpoint of the temporal programming of the process (injection, coating), the higher $x_{g e l}$ is, the longer is the period between the onset of polymerization and gelation. This is illustrated in Figure 21. The idea is also well illustrated in a conversion-temperature transformation (CTT) diagram. CTT diagrams are counterparts of TTT diagrams where the time axis is replaced by a conversion axis [2, 112]. A typical CTT diagram for a thermosetting polymer is represented in Figure 22. Similarly, the different states in which the system may be found are bounded by the gelation, vitrification and degradation curves. Clearly, if $x_{\text {gel }}$ is increased, then the area of the liquid region is increased, providing the operator with a better ability to handle the curing system.

Thus, once polymerization is underway two possible solutions are available to delay the gel point: (i) slow down the polymerization rate (via addition of retarders for instance) or (ii) increase the critical conversion.

Increasing the value of $x_{\text {gel }}$ may be beneficial to reduce the formation of internal stresses generated during the cure. Indeed, after the gel point the material loses its macroscopic mobility and the shrinkage, which is often observed after gelation, leads to the appearance of stress and formation of microcracks and microdeformations. When gelation appears at high critical conversions, curing mostly proceeds when the material is still able to relax the stress and the effect is limited [113, 114]. 


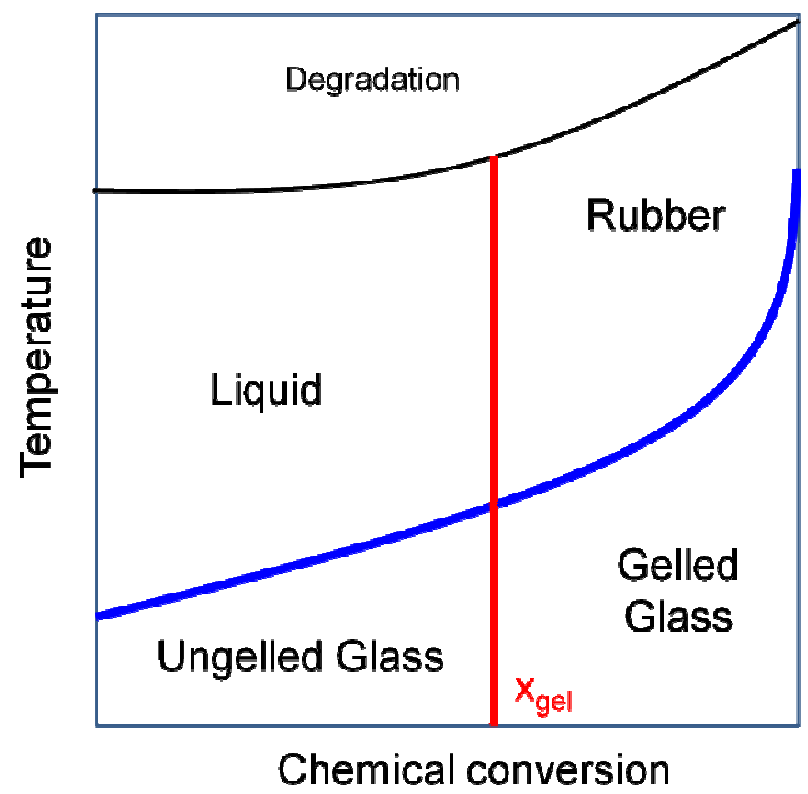

Figure 22: A typical conversion-temperature transformation (CTT) diagram for a thermosetting polymer.

\subsubsection{From monomer functionality to transfer agent}

Increasing the critical conversion is not simply a question of chemical reactivity. This implies the control of the chain growth process of polymerization. Stepwise and chain polymerization do not present the same degree of freedom to control the chain growth process. If one takes a look at the mathematical expressions to predict the gel point, it is evident that the critical conversions of chain and step polymerization are dictated by very different parameters.

(i) For stepwise polymerization, the statistical approach of Flory and Stockmayer permits to predict the extent of the reaction at the gel point $[2,72,115]$. For the ideal polymerization of $N_{\mathrm{A}}$ moles of a molecule A with a functionality $f_{A}>2$ (for instance $\mathrm{A}=$ diepoxy) and $N_{\mathrm{B}}$ mole of a molecule $\mathrm{B}$ with a functionality $f_{B}>2$ (for instance $\mathrm{B}=$ diamine), conversion at the gel point is given by

$$
x_{\text {gel }}=\left[r\left(f_{A}-1\right)\left(f_{B}-1\right)\right]^{-1 / 2}
$$

Where $r$ is defined as the ratio of the total number of functional groups of the limiting reagent to the total number of functional groups of the excess reagent (i.e. $r \leq 1$ ). Thus, for a system where the total number of A groups is less than the number of $\mathrm{B}$ groups, $r$ is given by: 


$$
r=\frac{N_{A} f_{A}}{N_{B} f_{B}}
$$

Similar expressions have been derived for mixtures containing two or more molecules with the same functional group but different functionalities (e.g. curing of a mixture of a triepoxy and a diepoxy with a diamine) or molecules with functionality equal or lower than 2 (e.g. use of a monoepoxy as a reactive diluent). They are very well reviewed in reference books $[2$, 98].

By following these expressions, it appears that only two parameters might be used to control the conversion at the gel point: (i) the functionality of the monomers (ii) and their stoichiometric ratio. For instance replacing a fraction of tetrafunctional monomers by difunctional monomers will result in an increase of $x_{g e l}$ [116]. But, at the same time, this will decrease the number of crosslink points and this could be detrimental for the properties of the final material. Similarly, a decrease of the stoichiometric ratio, $r$, will be accompanied by an increase of the conversion at the gel point but a fraction of functional groups will remain unreacted and the crosslink density will be lower than in the case of the stoichiometric mixture of the reactants [117].

(ii) For chainwise polymerization, the gel conversion depends in a complex way on the relative rate of initiation, propagation and termination, but also on the amount of initiator [77, 118]. Increasing the initiator concentration results in shorter primary chains and a corresponding increase in the gel conversion.

There is an extensive literature on the mathematical treatment of the crosslinking process. Regarding the specific case of epoxy resins, detailed studies have been reported for the homopolymerization in the cationic $[119,120]$ and the anionic [121] modes as well as the alternating copolymerization with cyclic anhydrides [54, 56, 122]. As in the case of step polymerization, the statistical approach of Stockmayer can be used to derive a simple and general expression of the critical conversion under the assumption of ideal chain polymerization. In particular, when one considers the reaction of a difunctional monomer $A$ (e.g. monoepoxy) with a tetrafunctional crosslinker $B B$ (e.g. diepoxy), the Stockmayer's equation predicts that the gel point is reached for [76]:

$$
x_{\text {gel }}=\frac{1}{\rho\left(\bar{X}_{w}-1\right)}
$$


Where $\bar{X}_{w}$ is the weight-average degree of polymerization produced by a single activation and a single termination step (i.e the weight-average degree of polymerization of the linear primary chains) and $p$ is the fraction of all functional groups residing on the crosslinker molecules. Thus if $[A]$ is the concentration of monomer and $[B]$ is the total concentration of functional groups of the crosslinker $([B]=2[B B])$, then $\rho$ is given by:

$$
\rho=\frac{[B]}{[A]+[B]}
$$

It is worth noting that the ring-opening polymerization of epoxies is rapidly outside the scope of the ideal conditions stated by Stockmayer. In particular, intramolecular cyclizations are rapidly observed through the mechanisms of back- and end- biting [76]. Moreover, when the first functional group of a crosslinker is reacted the reactivity of the second functional group is often affected [98]. For all these reasons, it is very difficult to verify Stockmayer's assumptions for high fractions of crosslinker $B B$. To take into account non-ideal phenomena (i.e. cyclization or reactivity ratio), it is necessary to introduce complex computational methods such as dynamic Monte Carlo simulation [123]. These more elaborated models will not be detailed here.

Despite the limitation of the statistical approach, it is interesting to note that, according to Eq. (3), a number of variables can be used to control the gelation process of an epoxy system cured by ring-opening homopolymerization:

- Gelation can be delayed by reducing the amount of crosslinker (i.e. decreasing $\rho$ ), but this is accompanied by serious modifications of the final properties of the material.

- One can reduce $\bar{X}_{w}$ by using a chain transfer agent (CTA). This is a very effective solution that will be further discussed in the case of the cationic polymerization of epoxy (See Section 4).

- One can also play with the reactivity of the functions of the crosslinker. If the reaction of the first function results in the decrease of the reactivity of the remaining function, then the gelation is markedly delayed. On the contrary, it is also possible to obtain extensive crosslinking with the rapid formation of tight network structure, by avoiding chain transfer and using high amounts of crosslinker whose functions have similar reactivity $[75,76,118,124]$. 
It is interesting to note that the effect of some CTAs may be accompanied by a decrease of the polymerization rate. For this reason, retarders are sometimes assimilated to CTAs. However, CTAs and retarders are different classes of additives [98]. True CTAs lower polymer molecular weights but do not affect polymerization rates. Retarders lower polymerization rates and polymer molecular weights.

In section 4 , the control of gelation through the adjustment of the critical conversion's value $\left(x_{g e l}\right)$ is reviewed in the case of epoxy thermosets. The discussion will be focused on the results observed in anionic and cationic ring-opening polymerizations. Particular attention will be paid to the cationic mode for which the use of alcohols as CTAs has received much attention through the extensive work reported by Penczek and coworkers [125].

\section{Control of the onset of the crosslinking process}

\subsection{Stepwise polymerization}

\subsubsection{Classical systems}

\subsubsection{Amines}

In step polymerization, the first approach to control reactivity of curing systems is to adjust the nucleophilicity of the hardener (amine, acid). Indeed, the pot-life of these twocomponents systems is ruled by the feasibility of one single reaction: the addition of one hardener function on one epoxy function.

For instance, aromatic amines are known to be less reactive than aliphatic ones due to their weaker nucleophilicity. Thus diaminodiphenylsulfone [126, 127] (DDS, Figure 23) can present pot-life of several months, while for diethylenetriamine [127] (DETA, Figure 23), it could hardly be longer than twenty minutes. As a counterpart, curing temperatures are much higher in the case of DDS and the curing process consumes more energy.

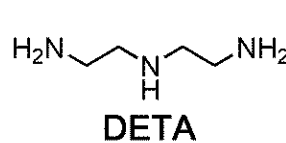

DETA

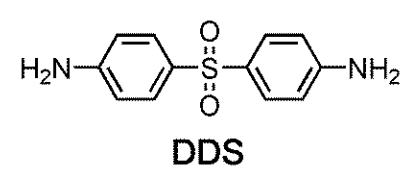

DDS

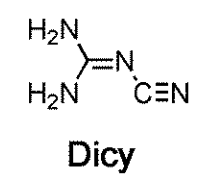

Figure 23: Structures of typical amine hardeners for epoxy resins. DETA is an alkylamine exhibiting no latency. DDS is an aromatic amine that reacts at high temperature and confers thermolatency to the curing system. Dicy is a non-soluble curing agent. Dicy is known to act both as an amine hardener and an initiator of anionic chain polymerization. 


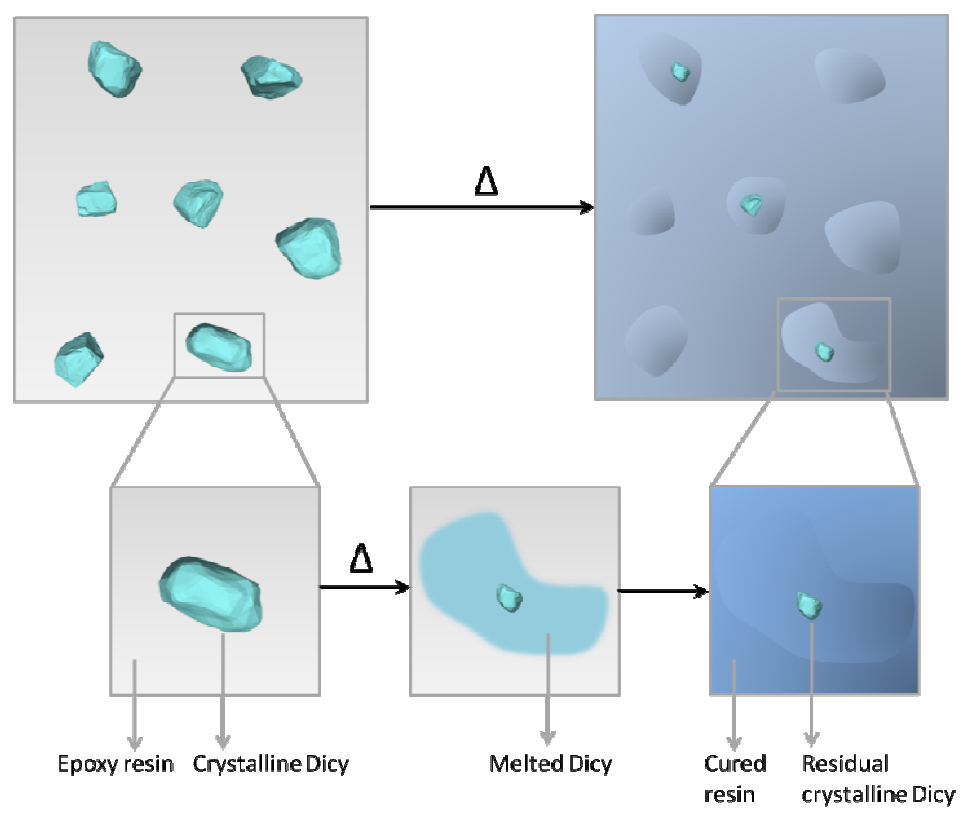

Figure 24: Control of the polymerization onset through a phase transition. This approach is typically used with DICY, a non-soluble curing agent that melts above $180^{\circ} \mathrm{C}$. The presence of residual crystal in the cured matrix might affect the properties of the network

Another typical solution to get latency is to use high melting point insoluble curing agents. Dicyandiamide [44, 128-132] (DICY, Figure 23), one of the most employed latent curing agents in epoxy technology, is a representative example. DICY has a pot life of 24 hours when it is dissolved in the resin with a solvent. But it is normally used in the form of fine powder dispersed in the resin. The resulting dispersion has a pot life of one year. As depicted in the scheme of Figure 24, polymerization starts at high temperature when DICY melts (typically $180^{\circ} \mathrm{C}$ ). Again, very high curing temperatures are necessary. Furthermore, the presence of residual crystals of DICY in the cured matrix may affect the mechanical behavior as well as the thermal performances of the resin [44].

It is interesting to note that, similarly, solid epoxy resins may be used to yield latent formulations. This approach is extensively used in coating technology with the "epoxy powder coating" process [15, 133]. A solid resin grounded in powder (usually a high molecular weight DGEBA) can be mixed in proper proportions with a powdered solid hardener (like DICY). They can also be combined in a fluid state, cooled down to a solid and grounded in powder. Under that form and as long as temperature is kept below $T_{\mathrm{g}}$ of the solid resin, reaction is prevented as a result of the immiscibility of the two components and/or the glassy state of the formulation that completely precludes the molecular motions necessary to observe a reaction. Upon application to a heated substrate the powdered system gains enough miscibility and mobility to react. This approach can be used to obtain very interesting 
formulation with long storage life at room temperature, ease of handling and no volatile materials. Furthermore, they may be cured at moderate temperature as the curing agent does not need to have a strong thermolatent behavior and can be selected to be active even at temperatures lower than the $T_{\mathrm{g}}$ of the resin [134]. However, it is essentially restricted to coating applications.

\subsubsection{Dihydrazides}

Other N-containing latent curing agents are dihydrazides. The typical chemical structure of this class of hardeners as well as the resulting reaction with epoxy functions are depicted in Figure 25 and Figure 26 respectively. They have been very scarcely described in the scientific literature but reports demonstrate they provide formulations with strong latent character in the thermal curing of DGEBA [135-137]. Interestingly, they are easily synthesized from the corresponding diacids and are available with very different chemical structures [40]. For instance, Tomuta et al. reported a comparative study of dihydrazides with aliphatic, cycloaliphatic and aromatic structures [135]. Like DICY, most hydrazides possess a high crystalline character and are dispersed as a solid in the liquid resin. Tomuta et al. observed a strong correlation between the melting point of the curing agent (usually $>180^{\circ} \mathrm{C}$ ) and the effective curing temperature. However, they also observed that a mixture of DGEBA with an amorphous and aliphatic dihydrazide exhibits a very high curing temperature as well. This is an important difference with aliphatic amines that usually react with epoxies at room temperature. It can be explained by the moderate nucleophicity of the $\mathrm{NH}_{2}$ groups of dihydrazides whose electronic density is severely reduced by the directly adjacent NH group [135]. Thus, dihydrazides are interesting alternatives to DICY or classical thermolatent aromatic amines (e.g. DDS), to yield latent epoxy formulation while being able to introduce flexibility to the resulting network.

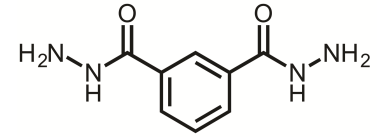

Isophtalic acid dihydrazide



Figure 25 : Structures of typical dihydrazides used to crosslink epoxy resins.

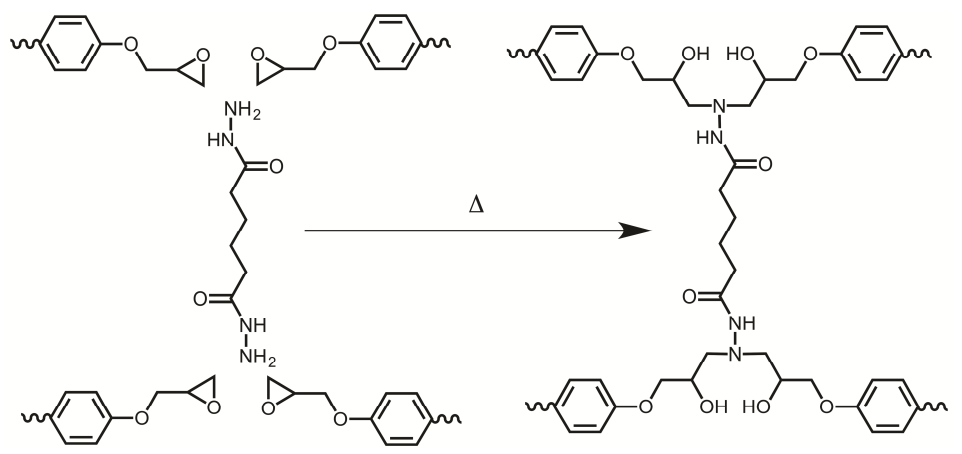

Figure 26 : Reaction of an aliphatic dihydrazide with four epoxy moieties. 


\subsubsection{Carboxylic acids}

Carboxylic acids form another class of curing agent that is extensively used in epoxy thermoset technology $[40,53,138]$. Their nucleophilicity is much lower than the above mentioned N-containing curing agents. Thus, the reactivity of homogeneous mixtures of epoxy resins and carboxylic acid hardeners is low enough to observe pot-lives of several months at room temperature [139]. However, for most applications, the reaction must be catalyzed to achieve acceptable reaction rate in reasonable temperature range [140, 141]. Then, pot-lives are controlled by the type and the concentration of the catalyst used. Both base and acid catalysis have been reported, but base-catalyzed conditions are usually preferred as the initiation of the competing homopolymerization of epoxies is much less in that case [140]. The base-catalyzed curing mechanism is illustrated in Figure 27. Among classical efficient base catalysts are: (i) imidazoles [142-144], (ii) tertiary amines [138, 145, 146], (iii) phosphonium bromide [140, 147] and (iv) some metal salts [80-82]. For instance, 1,2-dimethylimidazole [142] and dimethylbenzylamine [145] as well as tetrabutylphosphonium bromide [140] and zinc acetate [82] have been reported as very efficient catalysts (Figure 28). In any case, their use results in a drastic decrease of the potlives from several months to a few days. An alternative to obtain true latent curing formulations is the use of thermo- or photo- generated catalysts. They are inactive in normal storage conditions (room temperature and UV-blocking containers), and are liberated under stimuli such as high temperature or UV exposure. They include blocked tertiary amines [148, 149] and blocked imidazoles [150]. Most of these catalysts are actually used for the initiation of the ring opening homopolymerization of epoxy or the alternating ring-opening copolymerization with anhydrides. For this reason, they will be described in Section 3.2. It is worth noting that there are very few reports about the combination of carboxylic acids with true latent catalysts as carboxylic acids are mostly used in epoxy powder coating applications for which latency is insured by the solid state of the formulations [141, 151-153]. In that case, DICY is often used as the base-catalyst [152]. Typical carboxylic hardeners are carboxylic acid functional polyester resins (Figure 29). They provide materials with excellent corrosion resistance and adhesion which are very suitable for powder coating applications [40]. More recently, much attention has been devoted to bio-derived short-chain dicarboxylic acids (e.g. citric acid, Figure 29) and dimers or trimers of fatty acids (Figure 29) [154, 155]. 


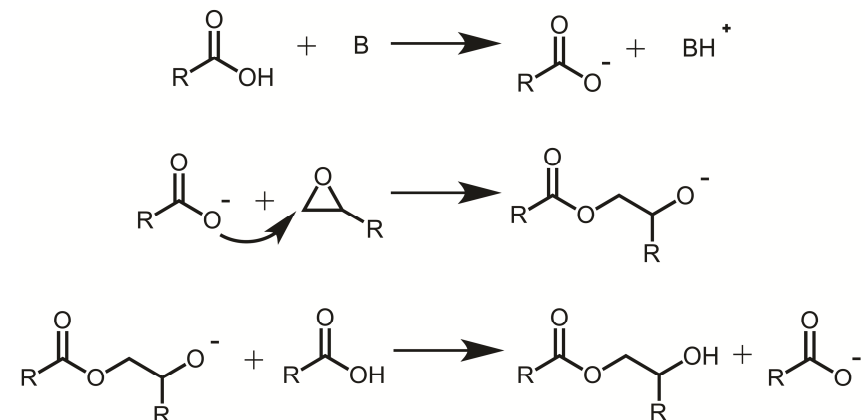

Figure 27 : Mechanism for the curing of epoxy resins with carboxylic acids under base-catalyzed conditions.

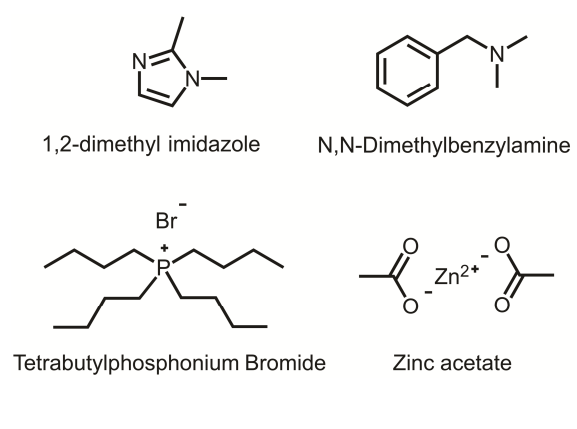

Figure 28 : Structures of typical base catalysts used for the curing reaction of epoxy resins with carboxylic acid crosslinkers.
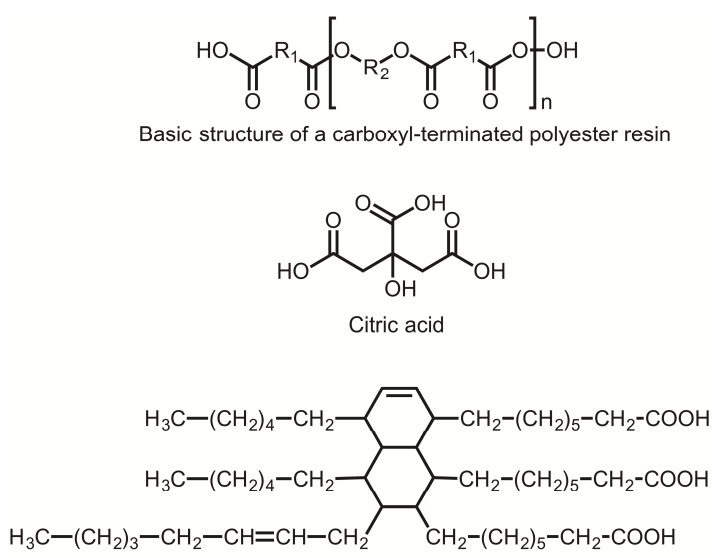

Trimer of fatty acid

Figure 29 : Structures of typical carboxylic acid crosslinkers used in epoxy thermoset technology.

\subsubsection{Thiols and isocyanates}

Other typical hardeners of epoxy resins are polythiols [156-159] and polyisocyanates [160163]. Like carboxylic acids, their nucleophilicity is usually much lower than amines or dihydrazides and base-catalyzed conditions must be used to observe adequate curing rates. Again, the stability of the catalyzed formulation may be insured by using latent catalysts such as thermo- or photo- generated amines (see Section 3.2.) [157, 161]. Typical thiol hardeners are polysulfides (Figure 30). They provide excellent adhesion properties [40, 164, 165]. A common isocyanate hardener is 4,4'-diphenylmethane diisocyanate (Figure 30) [160]. Isocyanate may be used when high $T_{\mathrm{g}}$ and good flame retardancy are desired. Indeed, the reaction of isocyanate with epoxy results in the formation of highly stable heterocyclic rings, namely isocyanurate rings (Figure 31$)[160,161]$. 


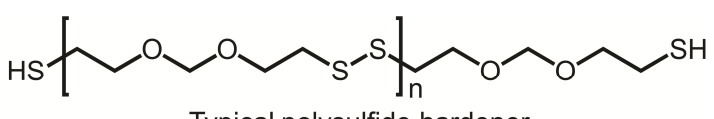

Typical polysulfide hardener

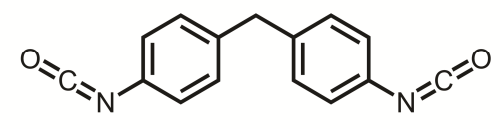

4,4'-diphenylmethane diisocyanate

Figure 30 : Structures of a typical polysulfide hardener and a typical diisocyanate hardener used in epoxy thermoset technology.

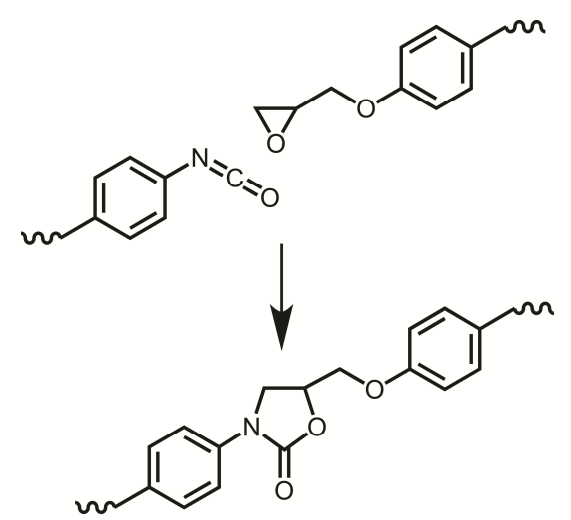

Figure 31 : Reaction of an isocyanate moiety with an epoxy moiety and formation of an isocyanurate cycle.

This overview of classical hardeners used in step polymerization is not exhaustive. However, one can easily identify the two main strategies to control the onset of the curing reaction in current industrial processes: (i) the use of poorly nucleophilic curing agents, (ii) the phase separation of the epoxy resins and the curing agents. Aliphatic amines seem automatically excluded from these strategies, precisely for the reasons that make them usually preferred: (i) low curing temperatures and (ii) the homogeneity of the curing formulations. Recently, new approaches have been proposed to confer latency to epoxy formulations using aliphatic amine in an effort to take advantage of their interesting properties.

\subsubsection{Encapsulated curing agents}

Instead of using a high melting point curing agent, another solution is to encapsulate the hardener so that it is efficiently separated from the epoxy in normal conditions but prone to diffuse outside the capsule when temperature increases. The principle of this approach is schematically illustrated in Figure 32.

Minami and coworkers use the so called Self-Assembly of Phase Separated Polymer method (SaPSeP) [166] in a dispersed aqueous medium to encapsulate an aliphatic diamine $(3,9-$ dipropanamine-2,4,8,10-tetraoxaspiroundecane) in a cured epoxy shell [167]. They obtain capsules with a load percentage of $43 \mathrm{wt} \%$ in unreacted diamine. The capsules are subsequently employed to formulate a one-component epoxy adhesive. To evaluate the storage stability of the system, the gelation time at room temperature was measured and compared with that of the ordinary epoxy-amine mixture. Whereas the ordinary system looses fluidity within 1 hour after mixing of epoxy and diamine, the one component system, with encapsulated diamine, retains its fluidity for at least 6 months. By heating the mixture at $70^{\circ} \mathrm{C}$ 
for 24 hours the authors observed the full curing of the adhesive pressed between two aluminium plates and measured a tensile shear strength of $2.5 \mathrm{MPa}$. Moreover, as the shell of the capsules is also made of epoxy, it is expected to chemically bind to the epoxy adhesive network due to unreacted moieties remaining on the particle surfaces. This method permits to use soluble aliphatic amines that is to say hardeners with a low curing temperature. Curing can be performed at moderate temperatures as long as the temperature is high enough to permit the release of the amine.

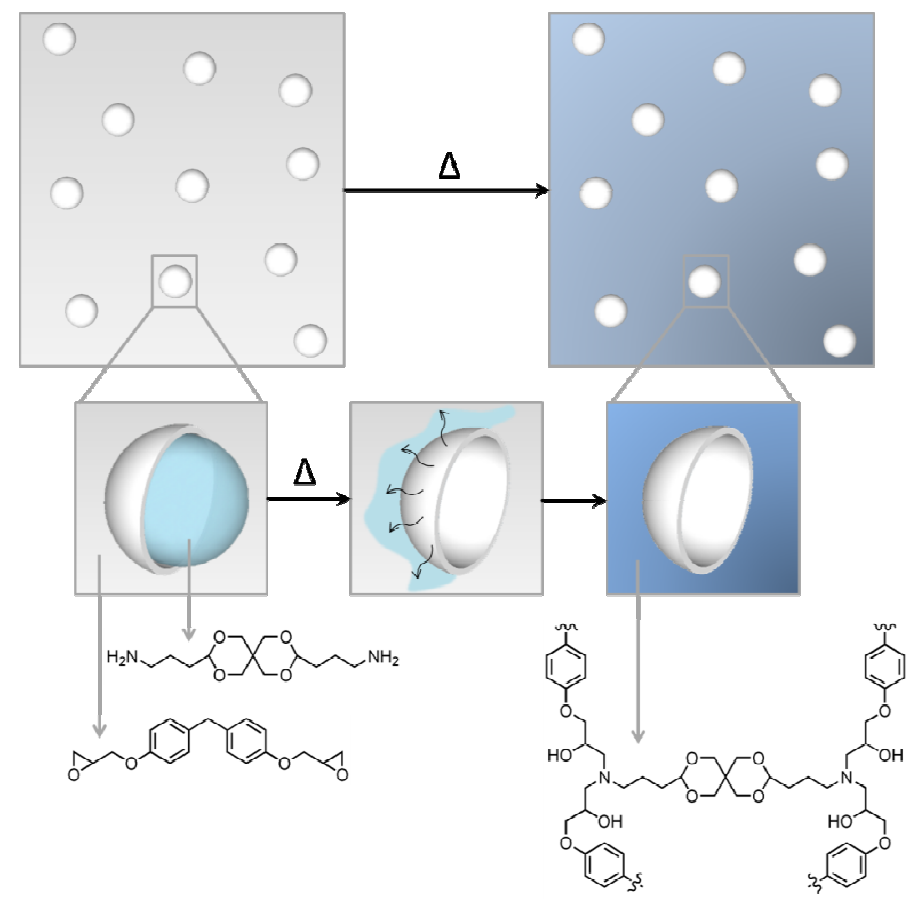

Figure 32: Schematic representation of the latent curing system proposed by Minami and coworkers (167). Capsules loaded with diamine are dispersed in an epoxy resin. Upon temperature increase the diamine diffuses outside the capsule and reacts with the diepoxy.

Inversely, Senatore et al. reported the encapsulation of a liquid epoxy crosslinker (namely an epoxidized linseed oil) to prevent its premature reaction with a carboxyl-functionalized polyester resin [168]. Again, the release of the resin is achieved by heating the system above the $T_{\mathrm{g}}$ of the polymeric micro-encapsulant (poly(N-vinyl)pyrrolidone). This strategy is used to devise new long shelf-life powder coating formulations.

A similar strategy has also been recently tested for the step-wise polymerization of epoxythiol formulations. Guzman and coworkers described the use of an encapsulated imidazole designed for one pot epoxy systems where a long pot life and a fast cure are required [156]. The capsule has a melting point at about $65^{\circ} \mathrm{C}$. Interestingly, this system offers a very good 
storage stability for temperatures as high as $35^{\circ} \mathrm{C}$ (pot-life of 3 weeks) while a very fast and efficient curing is observed at $120^{\circ} \mathrm{C}$.

It is very interesting to note that these systems are intimately bound to the field of self-healing polymers based on capsule rupture [169-171]. In that case, it is also highly desirable to control the time and place of the polymerization process. This is achieved through the use of microencapsulated polymerizable agents that are embedded in the material matrix and are released where and when it is necessary upon crack intrusion. The first successful self-healing composite exploited the polymerization of encapsulated dicyclopentadiene initiated by Grubbs' catalyst as the healing mechanism [171]. However, the high cost of the catalyst prohibits its widespread application. A cost-effective alternative was proposed by encapsulating liquid epoxy and amine hardeners in separate capsules, which mix and crosslink after the rupture of their respective capsules. The principle is schematically illustrated in Figure 33. Several methods were proposed by Moore et al. [172] and Studart et al. [173] to encapsulate amine hardeners. They were employed in healing dual microcapsule [172, 174176] systems and bode well for the use of this approach to control epoxy-amine step polymerization through phase separation.

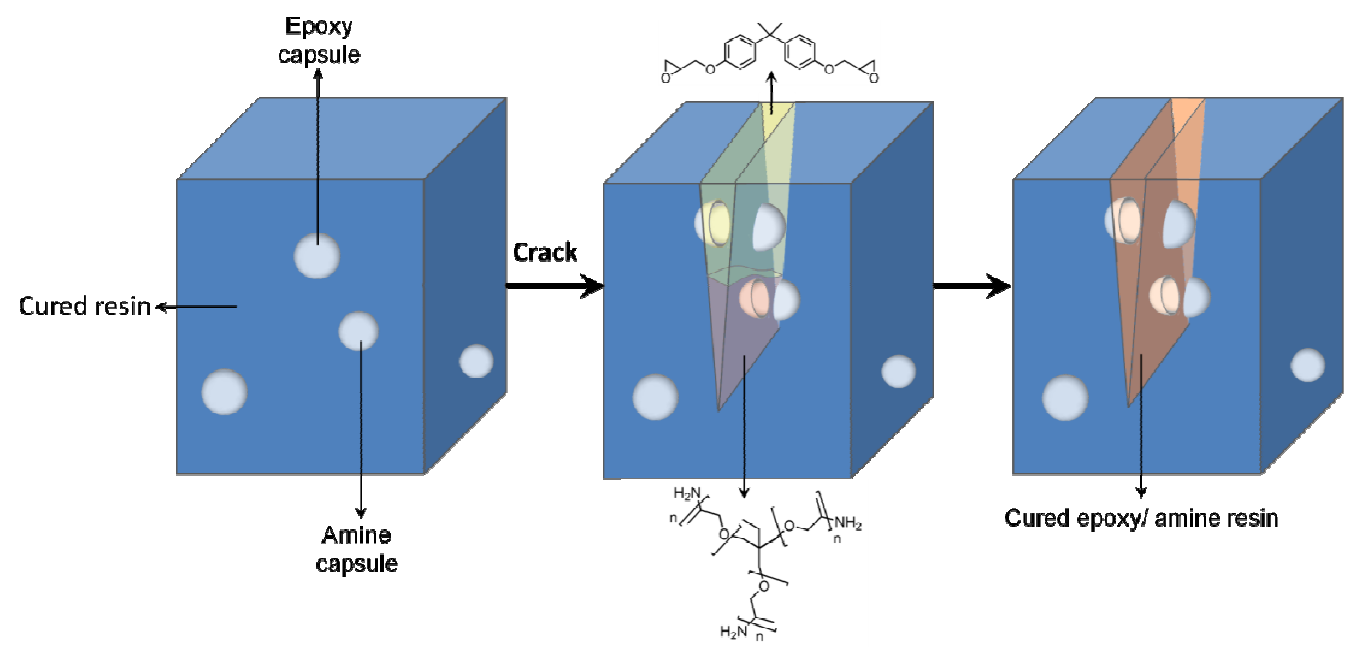

Figure 33: Epoxy-amine dual capsule healing system. Schematic representation of the system proposed by Jin et al. (175)

It should be remarked that others proposed the encapsulation of different hardeners, namely mercaptan with amine accelerator [177] or isocyanate [178]. Moreover, many methods have been described to control the release of chemicals in the field of drug delivery or fragrance [179]. It may be envisioned to use such strategies to control the curing of epoxy thermosets. 
Despite promising results in the field of self-healing materials, the potential of encapsulation to control crosslinking process must be moderated for several reasons. First, encapsulation is a more expensive approach in comparison to the use of insoluble latent hardeners like DICY. Moreover, in fiber resin composites the use of a biphasic system is a major drawback. Indeed, microcapsules will be severely inhomogeneously distributed during the impregnation process and the resulting matrix will not be cured properly.

\subsubsection{End capped curing agents}

To obtain a single-phase system with latent properties, several authors proposed chemically modified hardeners. The idea is to block the reactive functions of an hardener with a removable group. This strategy has historically been extensively used in polyurethane chemistry [180-183]. Figure 34 illustrates an example reported by Fréchet and coworkers. A molecule bearing one benzyl alcohol moiety and two isocyanate moieties blocked with aromatic alcohol was synthesized. Upon thermal treatment $\left(70^{\circ} \mathrm{C}\right)$, the isocyanate moieties are regenerated and react with the primary alcohol to form hyperbranched polymers.<smiles>[R]c1ccc(OC(=O)Nc2cc(CO)cc(NC(=O)Oc3ccc([R])cc3)c2)cc1</smiles>

Figure 34: Blocked isocyanate and the formation of a hyperbranched polyurethane by step-growth polymerization after thermal decomposition.
A<smiles>[R]N=C([R])CCCCCC([R])([R])O</smiles><smiles>[R]N[PH2+][CH]C([R])=O</smiles>

B<smiles>[R]N=C([R2])C([R3])[R]</smiles>

Figure 35: (A) Regeneration of amine by imine hydrolysis after exposure to normal atmosphere. The released amine can react with epoxy. (B) Tautomeric equilibrium between the imine and the more basic enamine. 
In the field of epoxy-amine thermosets, ketone-based imines have been developed as waterinitiated latent hardeners (Figure 35A). Indeed, imines are efficient protecting group of primary amines. The bulkier the ketone used to obtain the imine, the greater the steric repulsion between the epoxy and the nitrogen atom. Then, in storage conditions, ketimine's nucleophilicity is low enough to obtain mixtures with long shelf-life. When used in normal atmosphere as coating agent or painting, the system absorbs moisture that regenerates the amine by imine hydrolysis (Figure 35A). However, it must be emphasized that shelf-life is not infinite as ketimines are not completely unreactive towards epoxies due to the enamine-imine tautomerism presented in Figure 35B.

Endo et al., developed diethyl ketone-based imines showing fast hydrolysis rate in atmospheric conditions [47, 184, 185]. They observed that, despite the imine/enamine equilibrium, mixtures with epoxy resins present pot-life larger than 1 month. Thanks to their fast hydrolysis rate, these imines are used as hardeners for adhesives [184]. Endo and coworkers also show that it is possible to increase the curing rate of epoxy-imine mixtures by adding five-membered cyclic dithiocarbonates [186, 187]. Its reaction with an amine released from the imine results in the formation of a thiol group able to catalyse the amine-epoxide reaction. From the viewpoint of the adhesive properties, they report that the addition of methacrylate copolymer results in an improvement of the adhesive strength [188].

Takeyama et al. also proposed moisture-curable epoxy resins [189, 190]. They observe that the shelf-life stability and the curability of the compounds are mainly influenced by the steric hindrance of the ketimines. By using their system as an adhesive, they found that the lap shear strength is higher with a bulky ketone than with a small ketone as protective groups. This was attributed to the higher reaction conversion of the epoxide due to the larger plasticizing effect of bulky ketone. Takeyama and coworkers also reported a novel curing system based on a combination of urethane and epoxy resin cured with a moisture-latent hardener based on ketimine [191]. They compared ketimine and aromatic amine as latent curing agents and observed that using ketimine results in significantly higher fracture energies for this particular system.

In addition to the above-cited scientific literature, ketimines are now well established in the patent literature. However, this system is still inappropriate for a long storage period due to the difficulty of ensuring water free components and also to the unavoidable tautomeric equilibrium between the imine and the more basic enamine (Figure 35B). Moreover, it is 
essentially adapted to thin film applications because the regenerated ketone has to be removed through evaporation which is not without concern about VOC emission.

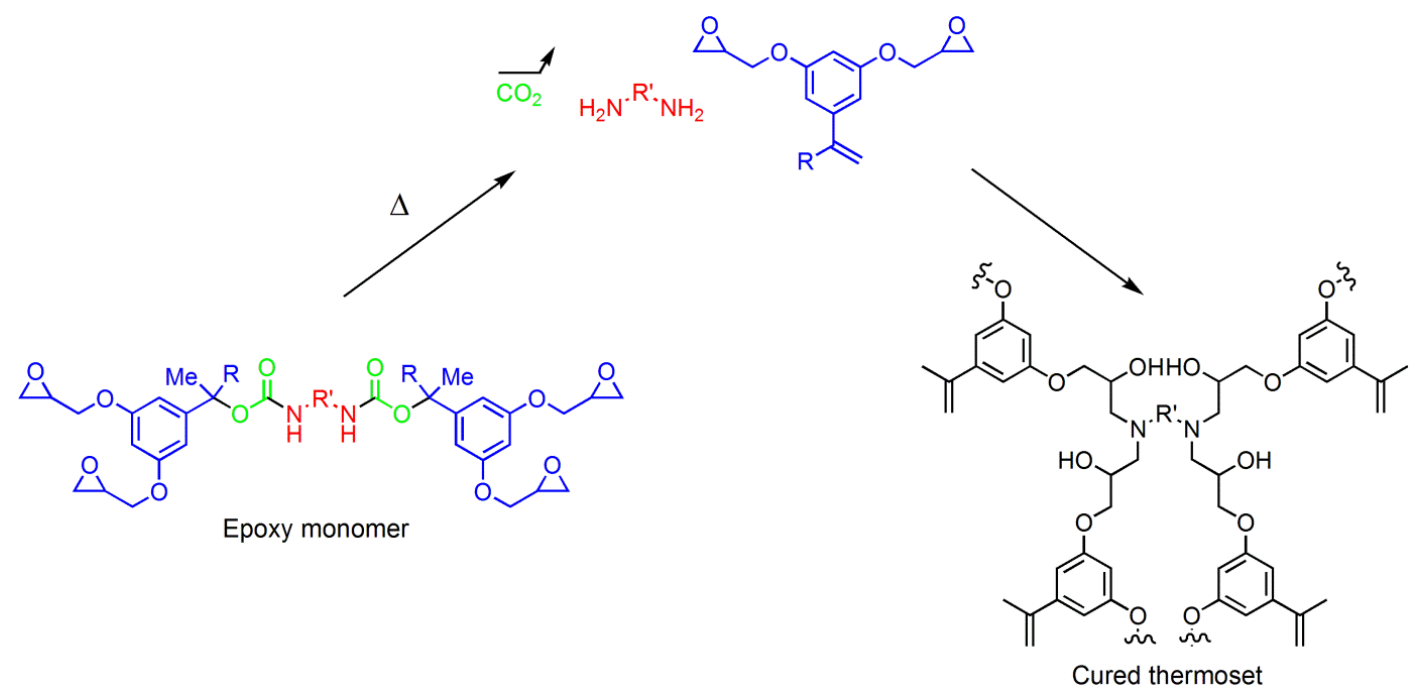

Figure 36: The single component epoxy system proposed by Fréchet et al. A diamine is held latent by thermally degradable carbamate linkages with multiple epoxide moieties. Upon high temperature exposure the carbamate decomposition yields an eliminated alkene (bearing epoxy moieties), a primary diamine and carbon dioxide, allowing the reaction of the amine with the epoxies.

Recently, Fréchet and coworkers proposed a thermally activated single component epoxy system in which the epoxy resin and the amine hardener are combined onto the same molecule [32]. The single molecule precursor contains both multiple free epoxide moieties and a diamine held latent by thermally degradable carbamates linkages (Figure 36). Thus, amines are blocked by the molecules bearing the epoxy moieties. The authors claim that the system presents an infinite shelf life. The onset of polymerization was observed for temperatures ranging from $170^{\circ} \mathrm{C}$ to $270^{\circ} \mathrm{C}$ (DSC measurements) depending on the structure of the carbamate linkages used.

The system takes advantage on the thermal decomposition of the carbamate functional group leading to an epoxy bearing alkene, a primary diamine and carbon dioxide. Thus, contrarily to ketimine curing agents, the byproduct here is a non-toxic gas. Fréchet et al. showed that the gas remains trapped within the film forming spherical bubbles with increasing size as the curing temperature increases. Such structures may be very useful as foam dielectrics, coatings, adhesives or sealants.

However, again, the byproduct resulting from the deprotection step clearly limits the scope of potential applications. Moreover, the thermal stability of carbamates results in very high curing temperatures comparable to those of classical latent curing agents (e.g. DICY). 
Interestingly, photo-generated amines protected under the form of carbamates [192-196] and O-acyloximes [197-202] have also been proposed as latent hardeners of epoxy resins.

In particular, amines have been protected under the form of 2-nitrobenzyl carbamates. Aromatic nitro compounds containing a carbon-hydrogen bond in the ortho position, with respect to the nitro group, are known to undergo intramolecular photoarrangement in which the nitro group is reduced to a nitroso group and an oxygen is inserted into the ortho benzylic carbon-hydrogen bond [193]. This mechanism is illustrated in Figure 37 for the case of a 2nitrobenzyl carbamates. It results in the release of a primary amine that might be used as a crosslinker for an epoxy resins [195].

When amines are protected under the form of O-acyloximes (Figure 38), the irradiation leads to the formation of an alkyl radical (2), an imino radical (3), and carbon dioxide. Then a ketimine (4) is formed by the recombination of 2 and 3, and finally an amine (5) and a ketone (6) are generated by the hydrolysis of 4 [203]. It is worth noting that UV exposure is not sufficient to recover the amine. The photo-generated product is a ketimine. As previously discussed, it must be hydrolyzed to yield the potent crosslinker, i.e. the amine. Many Oacyloximes have been described in the literature and used as photolatent crosslinker of epoxy resin [197, 199-202].

For both carbamates and O-acyloximes protected crosslinkers, the photogeneration of amine is accompanied by the release of two byproducts: a gas, $\mathrm{CO}_{2}$, that may be trapped and forms bubbles, and a small organic molecule (aldehyde or ketone) that might plasticize the network.
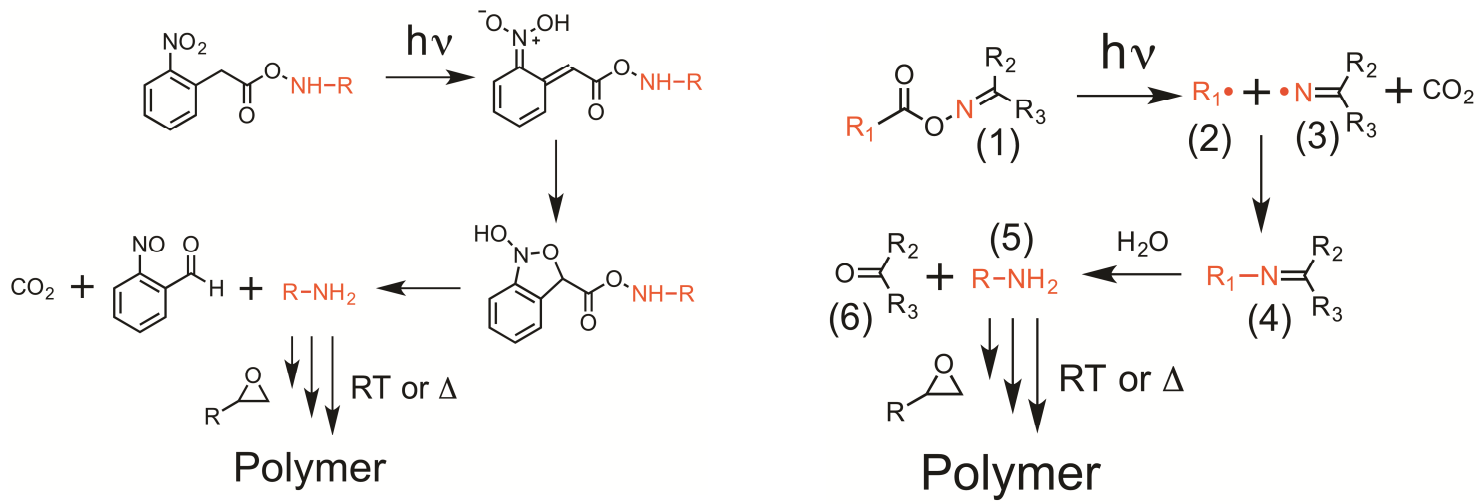

Figure 37: Photo-generation of an amine from a 2- Figure 38: Photo-generation of an amine from an Onitrobenzyl carbamates. acyloximes.

Amines blocked under the form of substituted ureas have also been used as thermolatent catalysts for the step-wise polymerization of epoxy-DICY [204, 205], epoxy-thiol [156, 206] and epoxy-isocyanate [161] formulations. For instance, Guzman and coworkers used N,N- 
dimethyl phenyl urea as a latent catalyst for the curing of DGEBA with a trithiol (trimethylolpropane tris(3-mercaptopropionate)) [156]. This alkylated urea is commercially available and it is expected to provide curing formulations with pot-life as long as 4 months (room temperature) according to the delivering company. The proposed mechanism to explain the latency is represented in Figure 39. At high temperature $\left(140^{\circ} \mathrm{C}\right.$ for $\mathrm{N}, \mathrm{N}$-dimethyl phenyl urea), the urea decomposes to release $\mathrm{N}, \mathrm{N}$-dimethylamine. This secondary amine reacts with an epoxide to form the corresponding tertiary amine that acts as the true catalyst of the epoxy-thiol step polymerization. Similar substituted ureas have been used by Endo et al. [204] as well as Poisson et al. [205] for the catalysis of the reaction between epoxy resins and DICY and by Pascault et al. [161] for isocyanate-epoxy systems. In these systems, it is worth noting that the protected amino function is not part of the crosslinker. It plays the role of a catalyst. A number of protected amines have also been reported as latent catalysts for the anionic ring-opening homopolymerization of epoxies. This will be discussed in Section 3.2. The reader should keep in mind that they might also be used to catalyze the step polymerization of epoxies with carboxylic acid, thiol or isocyanate crosslinkers.

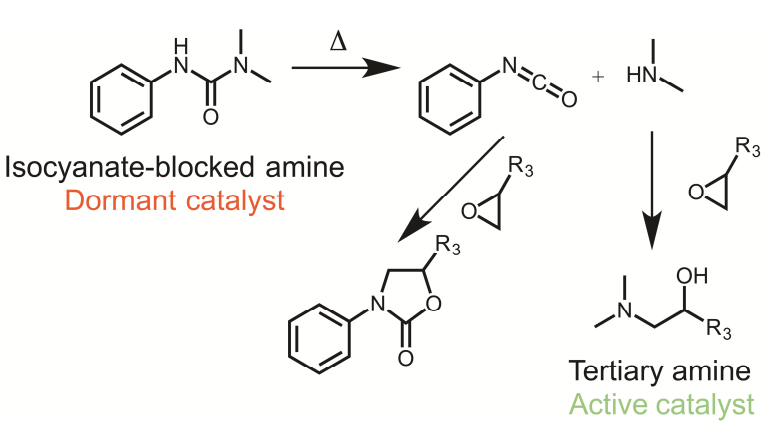

Figure 39 : Thermal decomposition of the N-aryl-N',N'-dialkyl urea used by Guzman et al. and release of a secondary amine. After reaction of the secondary amine with an epoxy moiety, the resulting tertiary amine acts as a catalyst of the thiol-epoxy reaction.
A - Hemiacetal ester synthesis

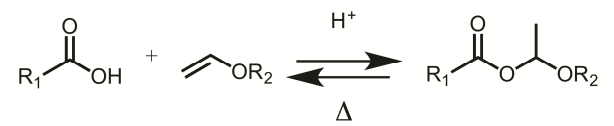

B - Thermal decomposition and reaction with epoxide Rate determining step

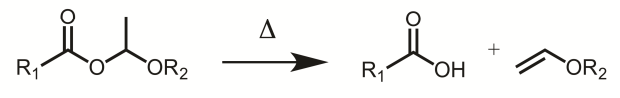
Addition with epoxide

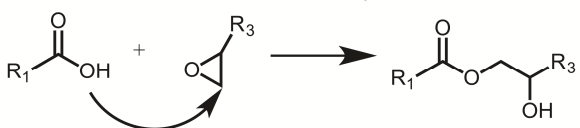

Figure 40: A : Hemiacetal esterification of a carboxyl group. B : Thermal dissociation of an hemiacetal ester and reaction of the deprotected carboxylic group with an epoxy moiety.

End capped carboxylic acids [207-212] and thiols [213,214] hardeners have also been used to design thermo- and photo- latent formulations with epoxies.

In a series of papers, Endo and coworkers examined the control of the thermal dissociation reaction of hemiacetal esters resulting from the reaction between carboxylic acids and alkyl vinyl ethers (Figure 40A) [215, 216]. They demonstrate that carboxylic acids may be released in a controlled fashion for temperature ranging from $150^{\circ} \mathrm{C}$ to $250^{\circ} \mathrm{C}$ by playing with 
chemical factors such as the bulkiness of the alkyl vinyl ethers. Similar thermal latent carboxylic derivatives were used to design epoxy resin formulations with high storage stability $[209,211,212]$. In these systems, the thermal dissociation of the hemiacetal esters is a rate determining step that permits to control the addition with epoxies as depicted in Figure 40B. Interestingly, this approach also increases the compatibility of the coexisting ingredients by suppressing the strong hydrogen bonding that normally exists in uncapped carboxylic acids, but again with release of a volatile organic compound.
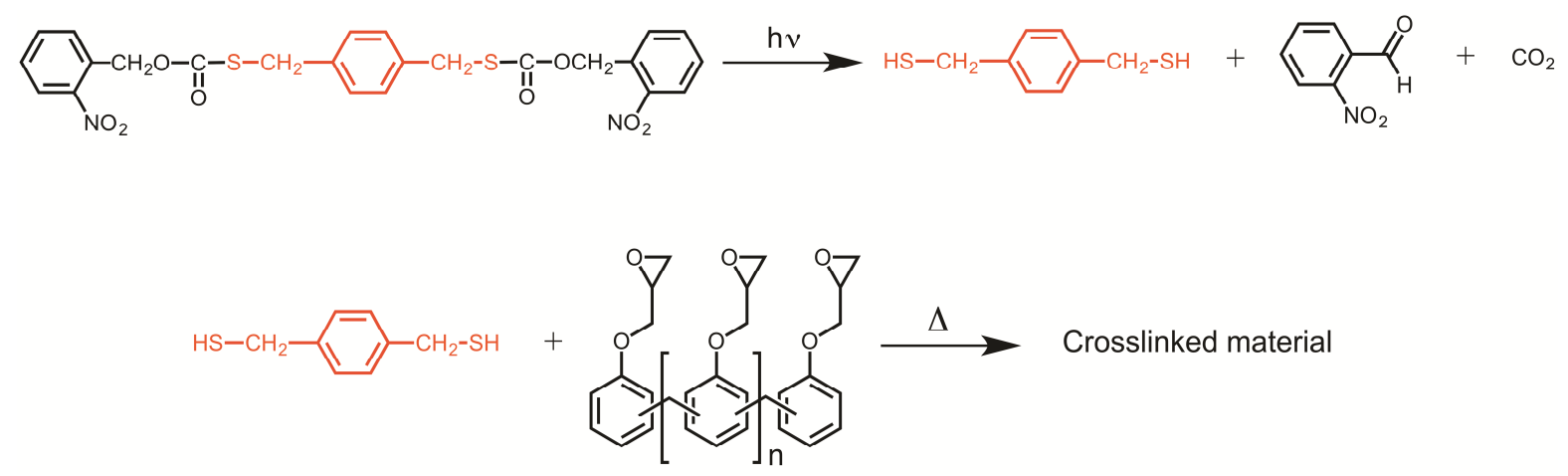

Figure 41 : Photo-generation of a difunctional thiol in a phenolic epoxy resin and subsequent thermal crosslinking of the system.

In the field of thiol-epoxy chemistry, a photo-cleavable crosslinker was described by Nishikubo et al [213, 214]. This system uses the photosensitive 2-nitrobenzyl moiety (Figure 41) already mentioned as a cleavable protective group for amine crosslinkers. Under normal storage conditions (room temperature, UV-blocking container), the slow reaction that is normally observed between free thiols and epoxides is completely precluded. Therefore, the pot-life of these formulations is very long. When desired, they can be cured according to a two-step process: (i) UV exposure to deprotect the thiols (ii) increase of the temperature to achieve acceptable reaction rates. Nishikubo used protected thiols in different epoxy resins, including polyphenol epoxy resins and poly(glycidyl methacrylate). They demonstrated that up to $80 \%$ of the thiol functions were deprotected after 60 min of UV irradiation. Similarly, up to $80 \%$ of the epoxy groups were consumed by heating at $120^{\circ} \mathrm{C}$ for 6 hours.

In the field of ring-opening polymerization, a large number of latent epoxy systems are available. Below, a description of the main approaches reported in the literature is proposed. It is focused on thermolatent and photolatent systems. Other external stimulations have been reported, notably high-energy irradiation [217-219], but their use is still very restrained and is not discussed here. 


\subsection{Anionic homopolymerization}

Tertiary amines have been extensively used as initiators of epoxy anionic homopolymerization. For instance imidazoles have long been recognized as highly effective, fast curing agents $[51,220]$. However, unmodified imidazoles have low stability when mixed with epoxies making them unsuitable for use in one-pot compositions. In the following section are presented the main strategies to design initiators able to deliver tertiary amines under thermal and light stimuli in a latent fashion as depicted in the schematic view of Figure 42. Particular attention is paid to recent development in the design of stabilized imidazole initiators. It is worth noting that tertiary amines are also key products in other curing processes. They are used as initiators of the anionic alternating copolymerization of epoxides with cyclic anhydrides (see Section 2.1.3) and they are essential catalysts for the step polymerizations with many hardeners including carboxylic acids and thiols (see Section 3.1.1). For the sake of conciseness, the following section is focused on the use of latent tertiary amines as initiators of the anionic homopolymerization of epoxides. But the reader must keep in mind that they might be employed to formulate other latent curing systems.

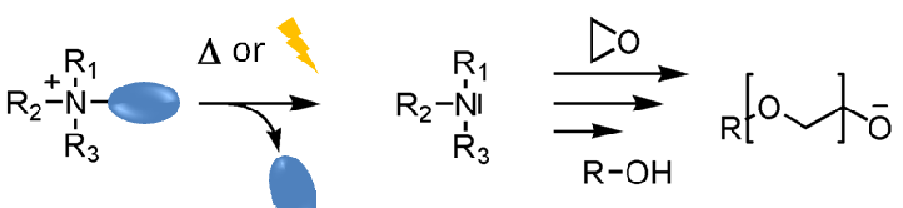

Figure 42: Schematic representation of the working principle of latent anionic initiators

\subsubsection{Thermogenerated amines}

Confronted to the low stability of imidazoles, several authors have proposed solutions to improve their workability by stabilizing the lone pair of the nitrogen atoms. One of the earliest strategies involves the preparation of metal-imidazole complexes. Hamerton and coworkers [221-223] reported considerable improvement of the stability of complexed imidazole initiators, with high onset temperature of polymerization (above $120^{\circ} \mathrm{C}$ ). However, most metal imidazole complexes are crystalline materials with very low solubility in common epoxies [221]. 


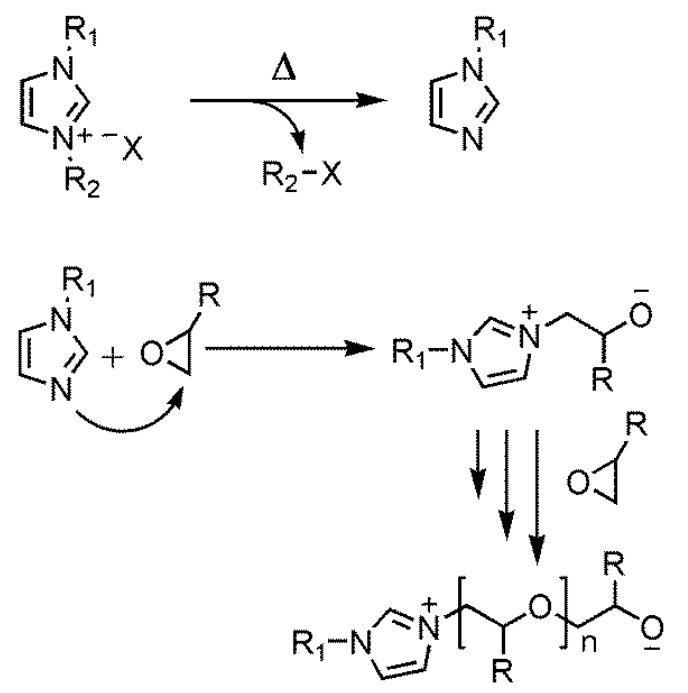

Figure 43: Proposed mechanism for the initiation of epoxy resin cross-linking with a 1,3-dialkylimidazolium: the decomposition of the imidazolium into an 1alkylimidazole is followed by the formation of a 1alkylimidazole/epoxy resin 1:1 adduct and the onset of anionic polymerization (classically $\mathrm{X}^{-}=\mathrm{Cl}^{-}, \mathrm{BF}_{4}^{-}, \mathrm{PF}_{6}^{-}$or $\mathrm{N}\left(\mathrm{CN}_{2}\right)^{-}, \mathrm{R}_{1}=$ methyl and $\mathrm{R}_{2}=$ ethyl, butyl or decyl)

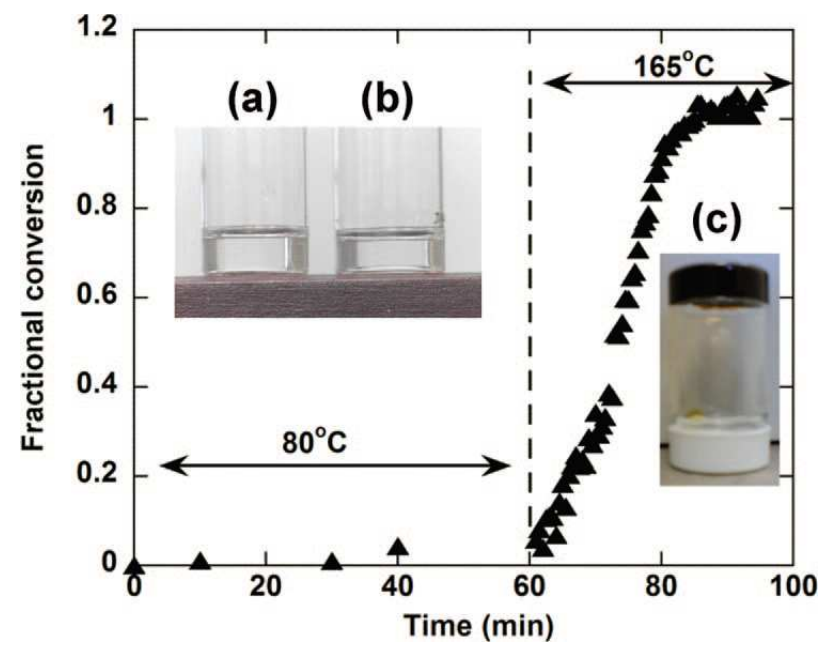

Figure 44: Plot of the epoxide groups conversion versus time showing the formulation of DGEBA with $3 \mathrm{wt} \%$ of 1-ethyl-3methylimidazolium dicyanamide at $80^{\circ} \mathrm{C}$ for $1 \mathrm{~h}$ followed by complete cure at $165^{\circ} \mathrm{C}$ within $20 \mathrm{~min}$. Inset (b) shows the same mixture after 60 days at room temperature with no apparent changes ((a) is the neat resin). Inset (c) shows the mixture after cure at $165^{\circ} \mathrm{C}$ for $2 \mathrm{~h}$ : a cross-linked and darkened sample is obtained (reproduced from (224) with permission of the American Chemical Society).

Joullié and coworkers [150] proposed the use of the 1,3-dialkylimidazolinium ion as thermolatent initiator. They showed that the quaternization of imidazole with a heat-labile group can block the active site effectively and extend the shelf-life considerably. They observed that, by heating above $200^{\circ} \mathrm{C}$, 1,3-dimethylimidazolium iodide liberates methyl iodide as a side product and 1-methylimidazole that is able to initiate polymerization. The mechanism is illustrated in Figure 43. The aim of this work was to overcome the problem of solubility and it paved the way to a current trend in the field of thermolatent anionic initiators: the use of ionic liquids.

Indeed, for the past few years, several authors proposed the use of commercial ionic liquid as latent initiators. In many cases, they are dialkylimidazolium ions that decompose at high temperature just like Joullié's system. Spychaj and coworkers first proposed the use of 1butyl-3-methylimidazolium tetrafluoroborate [225]. Recently, they showed that the reaction activity of various imidazolium cations, differing by the length of their alkyl chain and the nature of their counteranion, was related to their thermal decomposition characteristics [226]. They found optimal curing temperature ranging from $150^{\circ} \mathrm{C}$ to $200^{\circ} \mathrm{C}$. Palmese et al. [224, 227] proposed the use of 1-ethyl-3-methylimidazolium dicyanamide as an efficient thermolatent initiator. They observed pot-life at room temperature of more than 60 days. Curing operates in less than 20 minutes at $165^{\circ} \mathrm{C}$ as shown in Figure 44. Gérard and 
coworkers also described the influence of ionic liquid on the curing properties of several epoxy systems [228-230]. From all aforementioned solutions one has to remark the particularly high curing temperatures. This is undoubtedly due to the stability of the N-C bond that has to be broken.

Interestingly, Shin and coworkers [231-234] recently proposed the encapsulation of imidazole to afford latent curing systems. They observed shelf-life of more than 30 days at $20^{\circ} \mathrm{C}$ compared to 1 hour to 1 day for the free imidazole system [232]. Though elegant, this solution is unsuitable for many applications including the manufacture of pre-impregnated fiber composite materials.
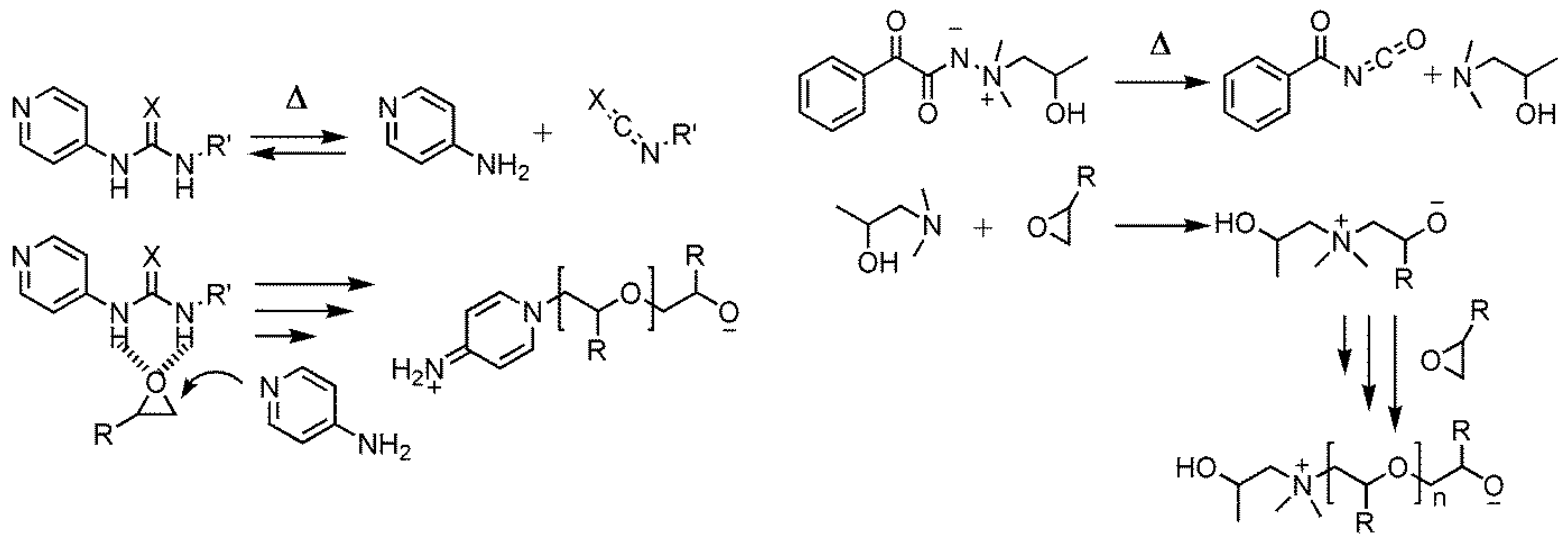

Figure 45: Plausible mechanism for the polymerization of epoxide using the urea $(\mathrm{X}=\mathrm{O})$ or thiourea $(\mathrm{X}=\mathrm{S})$ derivatives

Figure 46: Plausible mechanism for the polymerization of of 4 -aminopyridine as proposed by Endo et al. (235) epoxide using the aminimide proposed by Tomita et al

Concurrently, the group of Endo proposed several systems to release amine bases, other than imidazole, upon exposure to heat. They described a number of solutions all based on the cleavage of a covalent bond to liberate a secondary amine (piperidine [236-239], curing at $190^{\circ} \mathrm{C}$ ) or a tertiary amine (1,1-dimethylamino-2-propanol [148], curing at $\left.150^{\circ} \mathrm{C}\right)$. Very recently, they described the use of urea-derivatives of 4-aminopyridine [235]. They proposed a mechanism based on the thermal dissociation of the urea with possible involvement of some intact urea moieties in the acidic activation of the epoxy moieties. The postulated mechanism is presented in Figure 45. Curing systems are reported to be stable at $80^{\circ} \mathrm{C}$ and very reactive at $120^{\circ} \mathrm{C}$, while the system with the unprotected pyridine is reported to be reactive at $80^{\circ} \mathrm{C}$.

Again, the systems proposed by Endo and coworkers all require quite high temperatures to release the base and initiate the polymerization. High energy input is needed to break particularly stable covalent bonds. 
Recently Tomita et al., proposed a novel thermal latent anionic initiator for lower temperatures $[240,241]$. They reported a new class of aminimides that liberate 1,1dimethylamino-2-propanol upon heating at temperature as low as $80^{\circ} \mathrm{C}$. Chemicals formulas and the initiation mechanism are reported in Figure 46. The shelf-life is limited to 2 weeks in that case.

\subsubsection{Photogenerated amines}

Radiation curing is an interesting alternative to combine long shelf-life of ready-to-use formulations with lower curing temperature as compared to thermolatent systems. To this end, several authors have developed photobase generators [149], that liberate amines under suitable irradiation, and some of them have applied their systems to the photoinitiation of anionic polymerization of epoxies [242-245].

For instance, Chemtob and coworkers reported the use of several alpha-amino acetophenones that liberate tertiary amines upon irradiation. They used it in the simultaneous sol-gel and anionic photopolymerization of 3-(glycidyloxypropyl)trimethoxysilane [244]. Zeng and coworkers investigated a series of quaternary ammonium salts made of triethylenediamine bearing different chromophores. They were tested for the curing of a commercial DGEBA resin [243]. Figure 47 illustrates the photo-generation of triethylenediamine from the quaternary ammonium salt bearing the 4-acetylbiphenyl chromophore moiety.

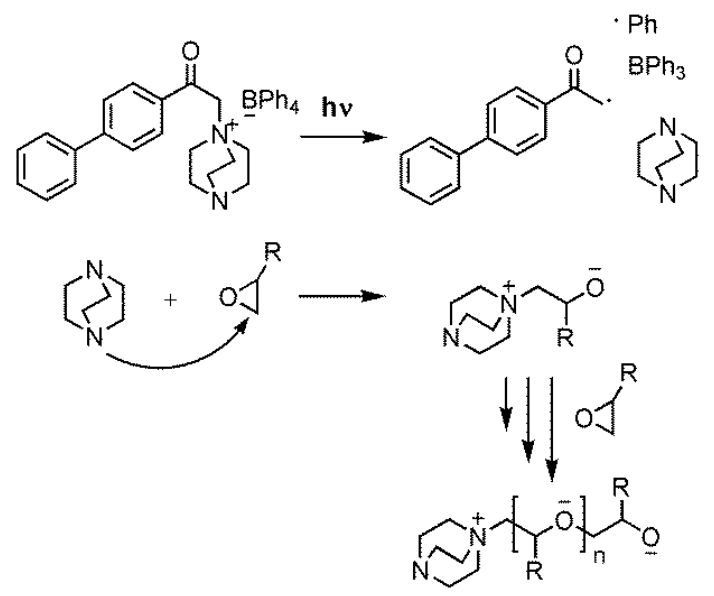

Figure 47: Proposed mechanism for the initiation of the anionic polymerization of epoxy in the presence of a photosensitive quaternary ammonium salt made of triethylenediamine and a 4-acetylbiphenyl moiety chromophore.
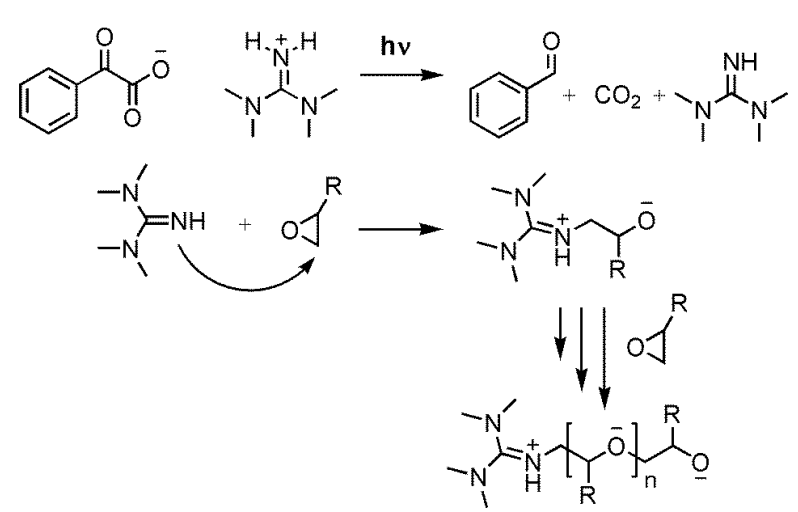

Figure 48: Photogeneration of a tetramethylguanidine molecule through hydrogen transfer between a tetramethylguanidinium ion and a decarboxylated phenylglyoxylate and subsequent initiation of the anionic polymerization of epoxy 
In both cases, the processes suffer from the low reactivity of the generated bases. They are much less active than imidazoles. Usually, UV-exposure must be accompanied by a postbaking step at high temperature [242-244] to overpass vitrification. Chemtob et al. subject their samples to a post bake at $120^{\circ} \mathrm{C}$ to observe a $75 \%$ yield conversion of the epoxy function. Zeng et al., heat their sample at $130^{\circ} \mathrm{C}$ for hours to observe complete epoxy conversion. Thus, these systems are not strictly speaking photolatent systems, but rather combine the characteristic features of photo- and thermo-latent systems.

Recently, Allonas and coworkers [245] described an original photobase generators with a hydrogen transfer between a tetramethylguanidinium ion and a decarboxylated phenylglyoxylate mediated by irradiation. It yields a tetramethylguanidine molecule with a strong basic character $(\mathrm{pKa}=13.6$, Figure 48$)$. Using this system, they observed the complete polymerization of an epoxidized hydrogenated bisphenol A resin at room temperature.

This last solution appears as a promising approach in the field of controlled anionic polymerization. However, it is restricted to the process of coating and thin films application due to the photo radiation dependence and byproducts evolution.

\subsection{Cationic homopolymerization}

Like in anionic polymerization, photolatent and thermolatent initiators have been described for the cationic polymerization of epoxy. Lewis acids, particularly $\mathrm{BF}_{3}$, are used for long time as initiators and activators in epoxy polymerization. Concurrently, in the 1970s, a new type of initiator based on organic onium salts was introduced. These concepts permitted the development of both photo- and thermo-latent initiators. Overall, the richness of the initiation chemistry dedicated to cationic polymerization greatly contributed to the substantial success of this polymerization mode in thermoset technology despite the historical hegemony of step polymerization.

\subsubsection{Lewis acid}

\subsubsection{Metal halides and triflates}

Lewis acids have long been reported as initiators of epoxide polymerization $[15,50,246]$. In particular, many metal halides are active initiators. They include: $\mathrm{AlCl}_{3}, \mathrm{SbCl}_{5}, \mathrm{BeCl}_{2}, \mathrm{FeCl}_{3}$, $\mathrm{FeBr}_{3}, \mathrm{SnCl}_{4}, \mathrm{TiCl}_{4}, \mathrm{ZrCl}_{4}, \mathrm{ZnCl}_{2}, \mathrm{BF}_{3}$ and $\mathrm{BCl}_{3}$ [15]. In the field of epoxy resin technology, they are usually considered as initiator of the cationic polymerization according to the 
mechanism illustrated in Figure 49. It is worth noting that the chain propagation is terminated by a $\mathrm{Cl}^{-}$transfer.

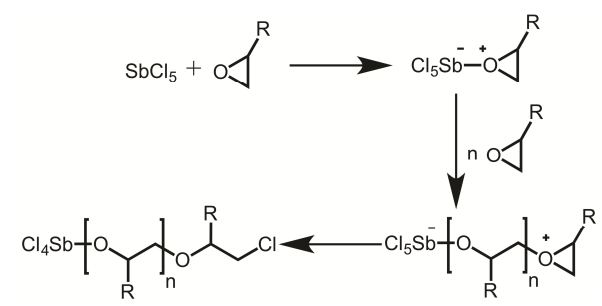

Figure 49: Mechanism of the cationic polymerization of epoxides in the presence of a Lewis acid $\left(\mathrm{SbCl}_{5}\right)$. Termination occurs by transfer of an halide anion from the metal to the activated chain end.

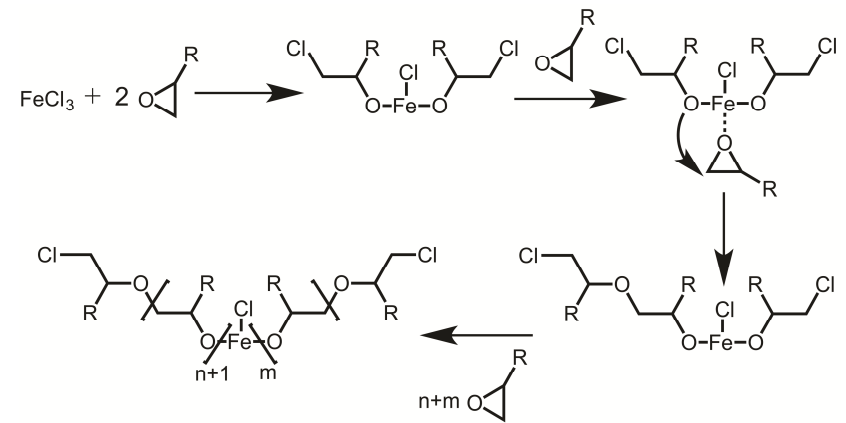

Figure 50 : Mechanism of the metal-mediated polymerization of epoxides in the presence of a Lewis acid $\left(\mathrm{FeCl}_{3}\right)$.

However, early reports demonstrated that the polymerization mechanism is highly complex and not necessarily well described in terms of cationic polymerization. In particular, in 1956, Price and Osgan observed that, if an optically active monomer (e.g. L-propylene oxide) is reacted with $\mathrm{FeCl}_{3}$, the optical activity is retained and the resulting polymer is stereoregular and crystalline [247]. Similar works demonstrated that several transition and post-transition metal halides might be used to synthesize crystalline and optically active polymers from monoepoxide monomers [248]. On the basis of these observations, a metal-mediated mechanism was proposed. It is illustrated for the case of $\mathrm{FeCl}_{3}$ in Figure 50. An initial reaction corresponds to the reaction of the metal halide with a few epoxy molecules to yield a product which may be represented as $\mathrm{ClFe}(\mathrm{OR})_{2}$ (in the case of $\mathrm{FeCl}_{3}$ ) where $\mathrm{OR}$ are alkoxide groups. Overall, this reaction presents the characteristic features of cationic polymerization. Experimentally, it occurs at room temperature but rapidly stops and polymerization is only restarted at higher temperature $\left(80^{\circ} \mathrm{C}-100^{\circ} \mathrm{C}\right)$, at a much slower rate. The mechanism for this second part of the reaction is thought to proceed by successive attacks of monomers by the alkoxides groups after their insertion in the coordination sphere of the complex. In this model, the mechanism should be considered as anionic as the active species are anion (alkoxides). These discoveries were the starting point of metal-mediated stereoselective epoxide polymerization. Since then, a wide variety of metal complexes were screened for epoxide homopolymerization and it opened up new horizon in epoxy chemistry, notably the stereoselective copolymerization with $\mathrm{CO}_{2}$ [249].

However, from the view point of epoxy resins' and thermoset's technologies, the cure cycles required to give optimum properties are usually too long with these complexes [15]. For this 
reason, to our knowledge, there is no report on resin systems using metal complexes based on transition or post-transition metals as initiator of the cationic polymerization.

In this context, only one Lewis acid has met commercial success in the epoxy resin technology: the metalloid halide $\mathrm{BF}_{3}$. The homopolymerization of epoxy initiated by $\mathrm{BF}_{3}$ is an extremely fast reaction that is very convenient to yield highly crosslinked materials [15, 40]. An extensive literature is devoted to the stabilization of $\mathrm{BF}_{3}$ with Lewis bases such as amines to form latent donor-acceptor complexes. This will be detailed in Section 3.3.1.2. Unlike the case of transition and post-transition metal halides, the polymerization mechanism has been established as cationic.

Other metalloid halides have been reported as efficient cationic initiator of epoxy polymerization. They include $\mathrm{SbCl}_{5}$ [246], $\mathrm{PF}_{5}$ [98] and $\mathrm{BY}_{3}$ where $\mathrm{Y}=\mathrm{Cl}, \mathrm{Br}$ or I [250, 251]. However, to our knowledge, they have not been used in epoxy resin's technology.

Recently, another class of Lewis acids has been proposed for the initiation of the cationic polymerization of epoxy resins: lanthanide triflates [252-255]. Interestingly, they combine a very acidic metal center (lanthanide) with a poorly nucleophilic anion (triflate). Therefore, the coordination of the oxiranic's oxygen with the metal is extremely tight and the proportion of chain-end processes is expected to be very low. Moreover, they are very stable compounds that are not moisture sensitive. The mechanism has been assumed to be cationic and propagation is described according to the equations reported in Figure 49. Given the very low nucleophicity of triflate, the anion is probably not involved in the termination step contrarily to chlorine or fluorine anions. Lanthanum (III), samarium (III), dysprosium (III), erbium (III) and ytterbium (III) triflates have all been successfully employed for the cationic curing of DGEBA [255]. Although the pot-life of formulations using these initiators has not been clearly discussed in the literature, the fairly high curing temperatures $\left(>100^{\circ} \mathrm{C}\right)$ reported in many different studies suggest that these initiators might provide reasonable pot-lifes. Moreover, they have been used with solid DGEBA resins to develop formulations suitable for powder coating technology [253].

\subsubsection{2. $\mathrm{BF}_{3}$-Lewis base complexes}

The boron trifluoride Lewis acid has been extensively used as a cationic initiator in epoxy polymerization. $\mathrm{BF}_{3}$ is usually stabilized by complexation with the lone pair of a Lewis base which can be an ether [256], an alcohol [257] or an amine [63, 69, 105, 258, 259]. Classical 
examples are illustrated for the three stabilization options in Figure 51. Due to their higher stability, $\mathrm{BF}_{3}-$ amine complexes are usually preferred. Several authors demonstrated that the reactivity of such complexes increases with decreasing basicity of the amine $[63,260]$. For instance, Pascault and coworkers observed that $\mathrm{BF}_{3}$-aromatic amine complexes do not show latency (aniline, $\mathrm{pKa}=4-5$ ). They readily initiate polymerization at room temperature. Concurrently $\mathrm{BF}_{3}$-amine, where the amine is aliphatic $(\mathrm{pKa}=9-10)$, hardly initiates the polymerization for temperatures below $100^{\circ} \mathrm{C}[63,261,262]$. Thus, by using aliphatic amines, it is possible to obtain thermolatent initiators. The complex of boron trifluoride and monoethylamine $\left(\mathrm{BF}_{3}-\mathrm{MEA}\right.$, Figure 51$)$ is still extensively used in large scale industrial applications including electrical insulating laminates and carbon reinforced plastics [263]. It gives very long pot-life formulations for temperatures below $100^{\circ} \mathrm{C}$. It is generally used at elevated curing temperatures comprised between $120^{\circ} \mathrm{C}$ and $180^{\circ} \mathrm{C}$.

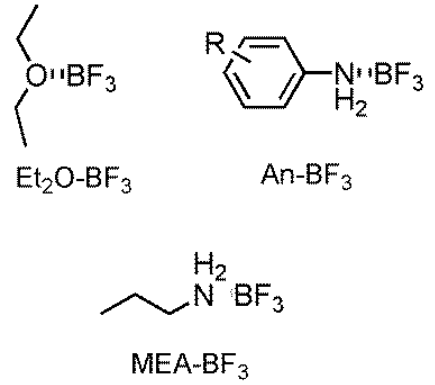

Figure 51: Typical $\mathrm{BF}_{3}$-Lewis base complexes used as cationic initiator of epoxy polymerization.

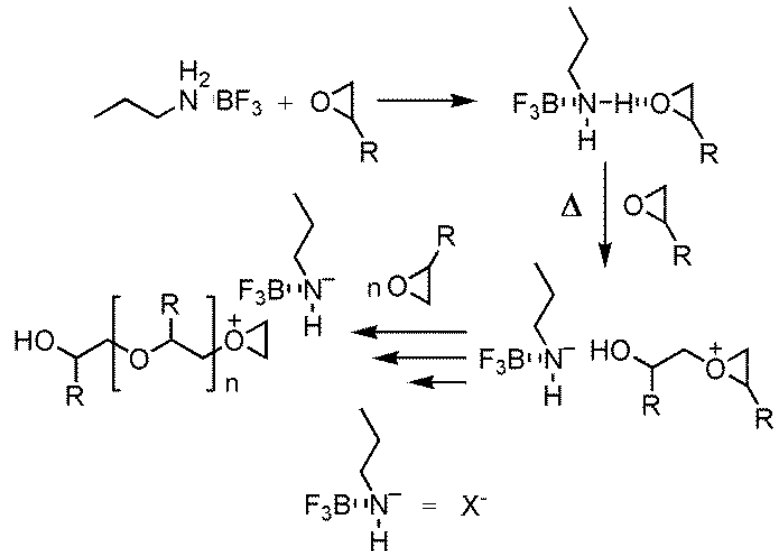

Figure 52: Initiation mechanism for the cationic polymerization of epoxy in the presence of $\mathrm{BF}_{3}$-amine complexes according to Harris and Temin (260).

The initiation mechanism of such complexes has long been discussed. Early models rely on the $\mathrm{BF}_{3}$-amine complex dissociation with heat, liberating $\mathrm{BF}_{3}$, that reacts with the epoxide according to the mechanism classically invoked for the cationic polymerization of epoxides initiated by Lewis acids (see Figure 49).

Harris and Temin [260] refuted the assumption of thermal dissociation of the complex. Indeed, they observed that $\mathrm{BF}_{3}$-amine complexes are thermally stable up to their sublimation temperature. Instead, they proposed a mechanism assuming the activation of the epoxy group through the formation of a weak hydrogen bond between the epoxy oxygen and a proton from the amine as illustrated in Figure 52. Eventually, the activated epoxy group is attacked by another nucleophilic epoxy group in a ring-opening step to give an activated chain end with 
the counteranion $\mathrm{X}^{-}=\left(\mathrm{RNHBF}_{3}\right)^{-}$. This mechanism was accepted and is still invoked by some authors [263]. However, the formation of the anion $\mathrm{X}^{-}$is hardly conceivable and several authors have disputed these assumptions. They showed that the truly active species is actually $\mathrm{HBF}_{4}$ or/and $\mathrm{HBF}_{3}(\mathrm{OH}) / \mathrm{HBF}_{2}(\mathrm{OH})_{2}$. In the absence of water, Tackie and Martin [264] invoked the thermal disproportionation of the $\mathrm{BF}_{3}$-amine complex through the transfer of one molecule of $\mathrm{HF}$, resulting in ammonium tetrafluoroborate and tetrafluoroboryl-amine. This option is represented in Figure 53. In the presence of water, Ghaemy [265, 266] as well as Smith et al. [267, 268], invoked the gradual hydrolysis of $\mathrm{BF}_{3}$ into borate as a potential source of $\mathrm{HF}$, which is able to generate $\mathrm{HBF}_{4}$ or one of its salts from $\mathrm{BF}_{3}$.

This last option appears as the most realistic. Furthermore, it is in accordance with classical initiating systems described in cationic polymerization of the carbon-carbon double bond. They were surprisingly eluded by aforementioned authors despite evident similarities. Indeed, initiation of carbon-carbon double bond polymerization with Lewis acid often relies on an initiator-coinitiator complex, also called syncatalyst system [98]. They consist in the association of a Lewis acid and a proton donor (a protogen). The most common initiating system combines boron trifluoride and water giving $\mathrm{H}^{+} \cdot \mathrm{BF}_{3}(\mathrm{OH})$. Figure 54 illustrates the formation of ammonium salts from $\mathrm{BF}_{3}$-MEA in the presence of water. This is in accordance with the observation of Smith et al., who reported the formation of $\operatorname{HBF}_{4}, \operatorname{HBF}_{3}(\mathrm{OH})$ and $\mathrm{HBF}_{2}(\mathrm{OH})_{2}$ when using $\mathrm{BF}_{3}-\mathrm{MEA}$ as an initiator of DGEBA polymerization.

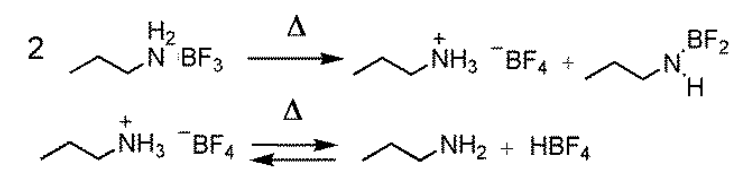

Figure 53: Thermal generation of tetrafluoroboron acid through the disproportionation of the $\mathrm{BF}_{3}$-amine complex according to Tackie and Martin.

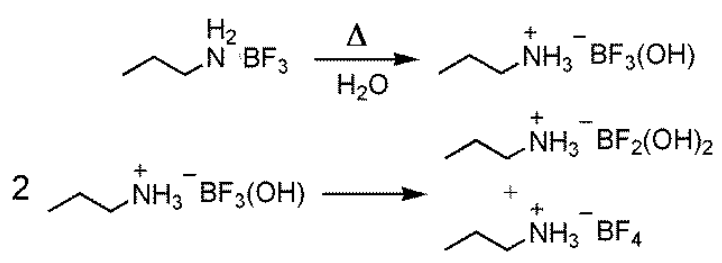

Figure 54: Thermal generation of tetrafluoroborate ammonium salt in the presence of water as a coinitiator.

Concurrently to $\mathrm{BF}_{3}$-amine complexes, $\mathrm{BF}_{3}$-ether and $\mathrm{BF}_{3}$-alcohol are very reactive complexes [257]. They readily crosslink epoxy monomers, even at low temperature. Again, it is interesting to note that several authors proposed to encapsulate this type of initiators to control their reactivity [256, 269-271]. Yong and coworkers encapsulate $\mathrm{BF}_{3}$-diethyl etherate in a tube structure for prolonging the shelf-life of an epoxy resin-based microelectronic packaging material [269]. Xiao et al. propose to use capsules loaded with the same initiator 
for the design of new self-healing materials. They described a two-components healing agent based on a cationic initiator and epoxy monomers loaded microcapsules [256, 270, 271].

\subsubsection{Onium salts}

From the historical standpoint, $\mathrm{BF}_{3}$-alkylamine adducts were the first thermolatent cationic initiators developed and applied in the field of epoxy polymerization. Concurrent efforts to design photolatent initiators of cationic polymerization have led to the development of another class of latent initiator: onium salts. First examples were reported in the 1970s and they have been the object of a particularly active research field since then. These salts are organic compounds containing a cationic heteroatom center with a metalloid fluoride anion. They mainly include iodonium, sulphonium, phosphonium and $\mathrm{N}$-containing onium salts. As for the systems previously reported for step and ionic polymerization, their latent properties are based on the release of active species subsequently to the cleavage of a covalent bond upon external stimulation.

With a few exceptions, the generic mechanism for the production of active species relies on a heterolytical or homolytical rupture of a carbon-heteroatom bond, leading to the formation of cations or radical cations, respectively. Figure 55 provides a schematic illustration of the generic mechanism. Polymerization is subsequently initiated by a carbocation or by a strong electrophilic Brønsted acid resulting from the reaction with a hydrogen donating solvent or a monomer.

Below are presented the main characteristics of the systems reported in the literature. Latent properties and curing conditions are discussed as well as recent developments of the field.

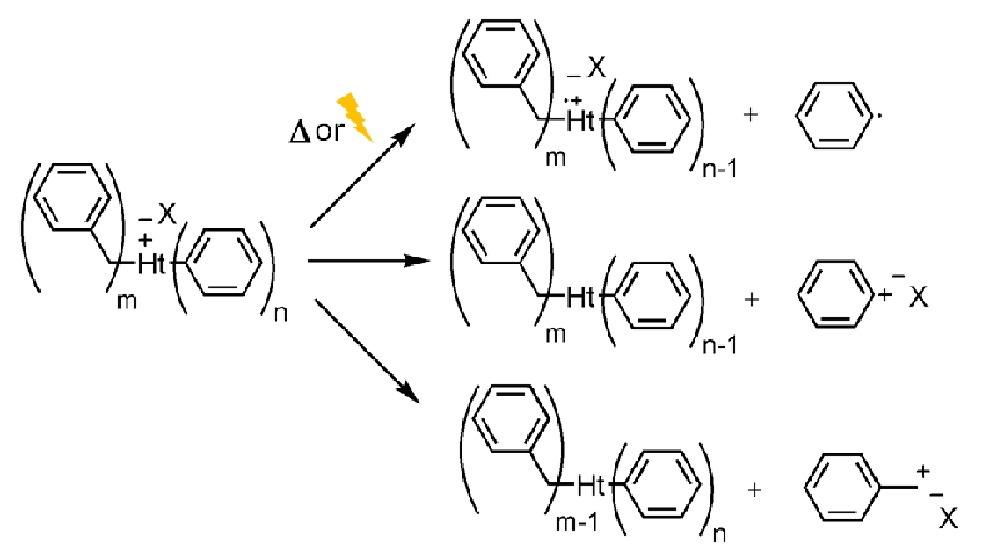

Figure 55: Typical heterolytic or hemolytic bond rupture that onium salts initiators undergo under exposure to heat or irradiation. $\mathrm{Ht}$ is a heteroatom $(\mathrm{Ht}$ $=\mathrm{N}, \mathrm{S}, \mathrm{P}$ or I), $\mathrm{X}^{-}$is a metalloid fluoride anion $\left(\mathrm{X}^{-}=\mathrm{SbF}_{6}^{-}, \mathrm{AsF}_{6}^{-}\right.$or $\left.\mathrm{PF}_{6}^{-}\right)$ 


\subsubsection{Photolatent onium salts initiators}

The real breakthrough in the field of onium salts cationic initiators was the description of iodonium and sulfonium salts as photoinitiators by Crivello and Lam [65, 66, 272-276]. Since then, a variety of onium photoinitiators have been reported in the literature with properties covering a wide spectrum in terms of thermal stability, solubility and efficiency to generate reactive species. Their typical chemical structures are illustrated in Figure 56. Detailed reviews specifically dedicated to this field are available [64, 277-279].

Among them, diaryliodonium compounds are most prominent due to their very easy preparation as well as high stability and reactivity. In their simplest form, they initiate polymerization under quite energetic UV radiation $(220-300 \mathrm{~nm})$. Their remarkable initiation efficiency and photosensitivity has contributed to their successful use in the polymerization of a large variety of epoxy monomers [218, 219, 278, 280-285]. Their initiation mechanism is representative of the general route to produce cationic species from onium salts and is described in Figure 57A.

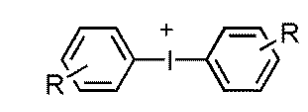

Diaryliodonium

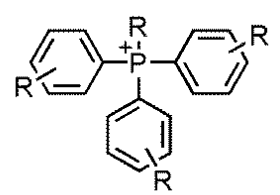

Triarylalkylphosphonium

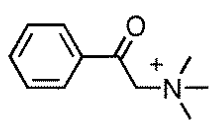

Trialkylphenacylammonium
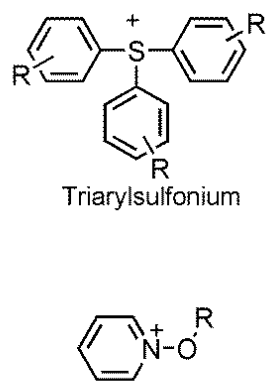

Alkoxypyridinium

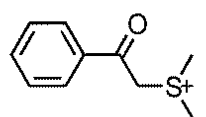

Dialkylphenacylsulfonium

Figure 56: Chemical structures of the main onium salts used as photoinitiators of cationic polymerization.

A
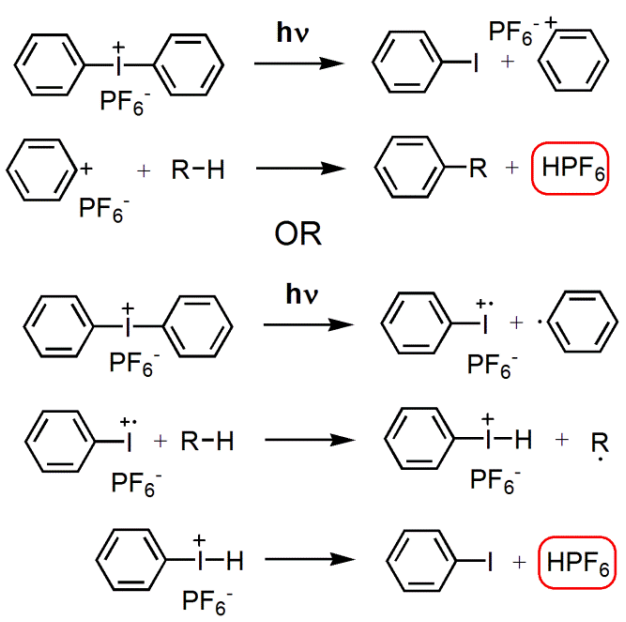

B

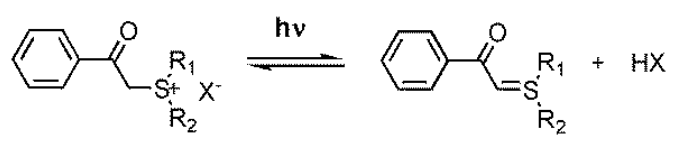

Figure 57: (A) Proposed mechanism for the generation of cationic species from iodonium salts under irradiation. RH may be the solvent or a monomer. (B) The overall mechanism resulting in the reversible photogeneration of acid by phenacylsulfonium salt.

Sulfonium salts have also been extensively used under their triaryl or alkylaryl forms. Like iodonium salts, they efficiently initiate the polymerization upon exposure to short- to midwavelenght UV light. Most of the time, they initiate polymerization through the formation of 
a strong Brønsted acid. But when used in their benzyl form, benzyl cations are the true initiating species and benzyl groups are incorporated into the growing chains. Their use has been reported in the polymerization of a number of epoxy monomers [108, 286-290].

Besides iodonium and sulfonium salts, phosphonium [291-295], pyridinium [296-302] or anilinium [303, 304] salts have been reported as effective photoinitiators for the cationic polymerization of epoxy. Most of them are aryl onium salts. However, alkoxypyridinium [299-301] and dialkylphenacylsulfonium [305, 306] are two exceptions that have considerably gained momentum over conventional aryl substituted salts. The former are remarkably soluble in most of the cationically polymerizable monomers. The latter undergo reversible photolysis so that it is possible to switch on and off the curing process through controlled UV exposure. The mechanism is schematically illustrated in Figure 57B.

Current investigations in this area are driven by the development of new initiating systems working under visible light [307, 308]. Indeed, systems with low energy requirement have become a matter of concern in the scope of green chemistry. However, most of the simple aryl onium salts undergo photolysis for wavelengths below $300 \mathrm{~nm}$ (UV). Several strategies have been proposed for improving the performance of these initiators in the near UV and visible spectral regions. They include (1) the direct modification of the initiator structure by introducing chromophoric groups on their aromatic rings [309, 310] or (2) indirect activation by combining the onium salt with additives.

These additives can be free radical photogenerators. They yield electron-rich donors upon visible light that are able to reduce onium salt. The radical induced decomposition of the onium salts is accompanied by the production of active cations [311, 312]. For instance, Durmaz et al. demonstrated that it is possible to observe a very efficient curing of epoxy monomers at $400 \mathrm{~nm}$ by combining a iodonium salt, that does not absorb at wavelength larger than $340 \mathrm{~nm}$, with the benzoyltrimethylgermane, an efficient free radical photoinitiator for wavelength as long as $425 \mathrm{~nm}$ [313]. The adsorption spectra of the two initiators are compared in Figure 58A. The initiation mechanism proposed by Durmaz and coworkers is presented in Figure 58B. It is based on the photogeneration of germyl radicals and benzoyl radicals in a first step. Subsequent oxidation of germyl radicals by onium salts yields germanium cations capable of initiating the cationic polymerization of epoxy monomers. 
A

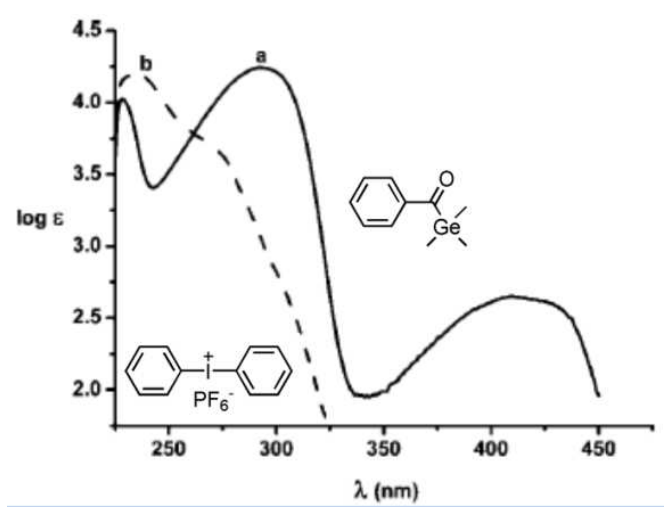

B

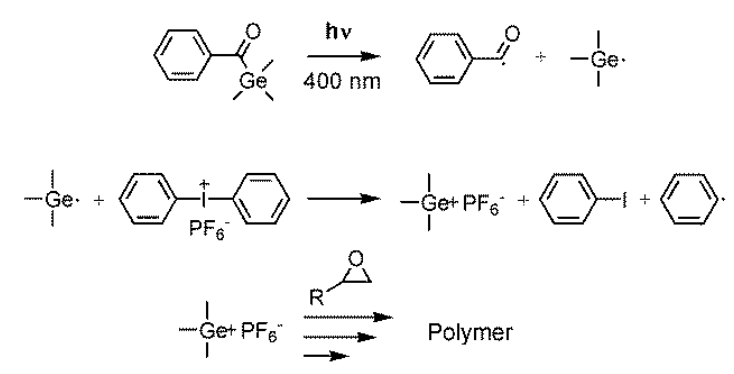

A

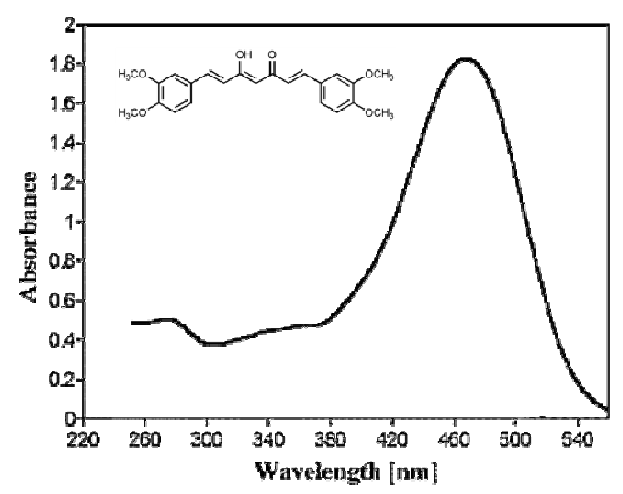

B

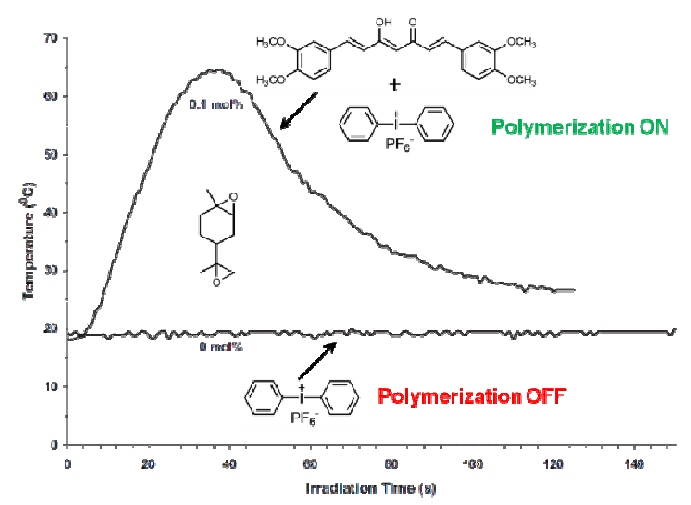

Figure 59: (A) Optical absorption spectra of curcumin (3.10 ${ }^{5} \mathrm{M}$ in basic aqueous solution $(\mathrm{NaOH} 0.5 \mathrm{M})$ (B) Study of the photopolymerization of limonene-dioxide with $1.0 \mathrm{~mol} \%$ of diaryliodonium salt in the absence of a photosensitizer and in the presence of $0.1 \mathrm{~mol} \%$ of curcumin (light intensity 395 $\mathrm{mJ} / \mathrm{cm}^{2}$ min, wavelength $407 \mathrm{~nm}$ ) (reproduced after (314) with permission from Wiley)
Figure 58: (A) Optical absorption spectra of benzoyltrimethylgermane (BTG) and diaryliodonium salt in $\mathrm{CH}_{2} \mathrm{Cl}_{2}$ (reproduced after (313) with permission American Chemical Society) (B) Photoinitiation of the cationic polymerization of epoxy by reduction of diaryliodonium salt in the presence of photogenerated germyl radicals (free radical promoted cationic polymerization) at $400 \mathrm{~nm}$.

A second mode of indirect activation of onium salts for long wavelength initiation is based on the use of photosensitizers. These are polynuclear chemicals able to absorb energy from visible light and transfer this energy to the onium salt (energy transfer mechanism) [301] or electron-rich donors that bind to the onium salts to yield complexes with absorption wavelength extending to the visible light (charge transfer mechanism) [315, 316]. For instance, Crivello and Bulut proposed to use curcumin, an intensely yellow dye isolated from Indian turmeric, as an electron-transfer photosensitizer [314, 317]. The chemical structure as well as the adsorption spectrum of curcumin are presented in Figure 59A. The molecule, that exists almost completely in the hydrogen bond-stabilized enol form, is strongly conjugated and adsorbs from 330 to $500 \mathrm{~nm}\left(\lambda_{\max }=427 \mathrm{~nm} ; \varepsilon=55000\right)$. Figure 59B illustrates the 
exothermic profiles as a function of irradiation time for the photopolymerization of limonene dioxide initiated by a diaryliodonium salts in the presence and in absence of curcumin under a wavelength of $407 \mathrm{~nm}$. In the absence of curcumin, photopolymerization was not observed even after $500 \mathrm{~s}$. In contrast, when curcumin is present, an exothermic peak with a maximum temperature of $65^{\circ} \mathrm{C}$ is indicative of immediate polymerization. It is consistent with a strong photosensitization effect.

Following these strategies, elegant green systems have been proposed [318-325] such as the polymerization of renewable epoxy monomers under sunlight [319].

This is contributing to make photolatent onium salts more and more attractive. Today, they are among the most prominent options to control the chain polymerization of epoxy monomers. They offer extremely long pot-life providing that the solutions are stored in the dark. But again, these appealing properties are balanced by the wide range of applications they cannot embrace including molding and injection. In the mean time, they are extremely attractive candidates in more recent technologies like 3D-printing [326].

\subsubsection{Thermolatent onium salts initiator}

Most of the onium salts described in the previous section may actually be activated thermolitically too. Some of them are too stable though, and many researches have been devoted to the adjustment of their pattern of substitution to observe bond rupture in a reasonable temperature range. Typically, a thermolatent onium salt contains a benzyl moiety. Common benzylsulfonium, benzylammonium and benzylphosphonium salts are illustrated in Figure 60. As depicted in Figure 61, they may release an initiating species in the form of a carbocation (Figure 61A) or in the form of a proton stemming from their benzylic methylene group (Figure 61B). 

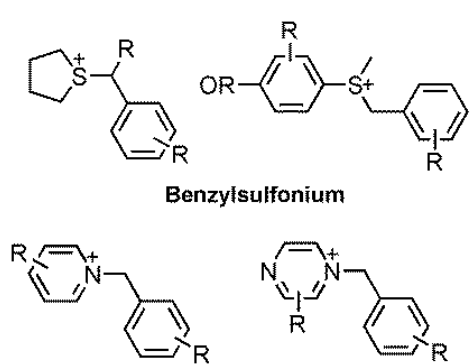

Benzylpyridinium
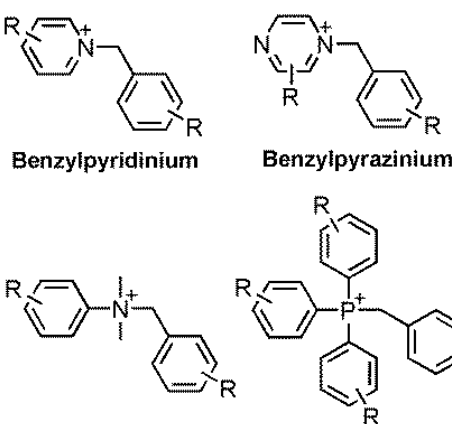

Benzylanilinium
Benzylpyrazinium

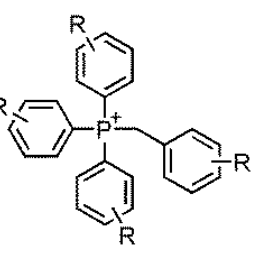

Benzylphosphonitum

Figure 60: The most common benzyl onium salts used as thermolatent initiators of cationic polymerization.

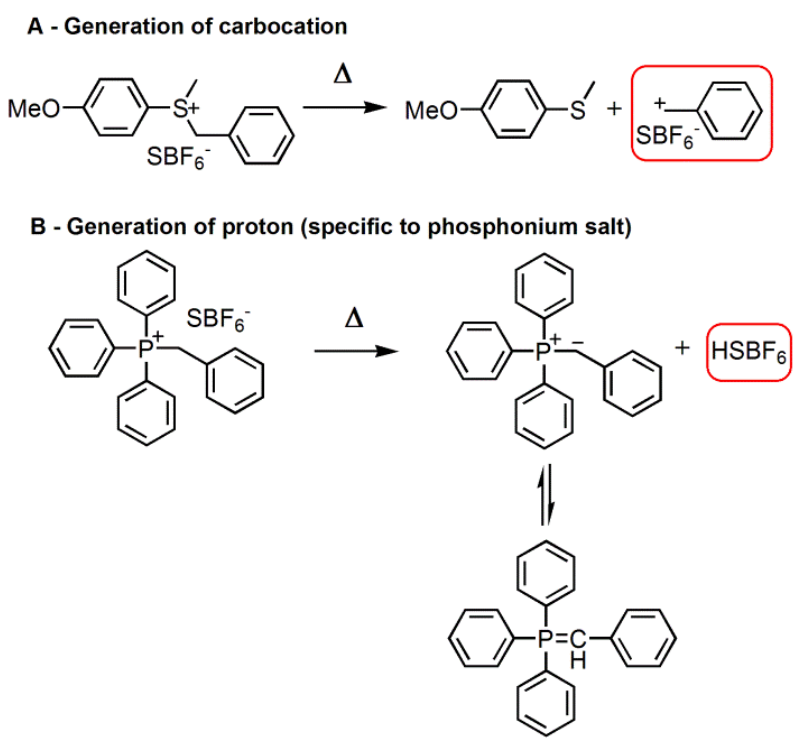

Figure 61: The two typical initiation mechanism of cationic polymerization with a thermolatent benzyl onium salt.

Papas and Hill [327] first reported the use of sulfonium salts as thermolatent cationic initiators. They established a basis for the rational design of latent thermal initiators with the help of relevant kinetic parameters to maximize stability while minimizing the curing temperature. Indeed, the ultimate goal of thermolatent initiation is to achieve high latency in a reasonable temperature range. In the language of chemical kinetics, this means that the system should have both high activation energy $E_{a}$ and high frequency factor $A$, in its Arrhenius equation (Eq. (5)).

$$
\ln (k)=\ln (A)-\frac{E_{a}}{R T}
$$

When $E_{a}$ increases, the threshold temperature corresponding to the cleavage of the initiator and the beginning of the polymerization increases too. However, according to the Arrhenius equation, this is accompanied by a decrease of the reaction rate constant $(k)$ and a sluggish polymerization, which is detrimental to practical use. A concomitant increase of the frequency factor, A, may compensate the decrease of $k$. Thus, according to Papas et al. the latent performance of a series of thermal initiators may be estimated and classified through the comparison of their respective $E_{a}$ and $A$ factors.

Following this work, the group of Endo and coworkers has been the major contributor to the field of latent thermal initiators over the last three decades. They demonstrated that several sulfonium [328-336], phosphonium [67, 68, 337-340], pyridinium [296, 298, 341-344] and other N-containing onium salts [345-347] could serve as latent thermal cationic initiators for 
epoxy polymerization. They observed that their initiating activity could be efficiently controlled through the variation of the electronic and steric properties of their substituents.

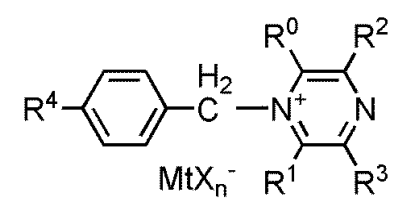

Table 1: Structure of various benzyl pyrazinium salts investigated as thermolatent cationic initiator in the polymerization of glycidyl phenyl ether (GPE). Influence of the benzyl and pyrazine substituents are under consideration as well as the influence of the counteranion.

\begin{tabular}{|c|c|c|c|c|c|c|c|c|c|}
\hline & 1 & 2 & 3 & 4 & 5 & 6 & 7 & 8 & 9 \\
\hline $\mathrm{R}^{\mathrm{O}}$ & $\mathrm{H}$ & $\mathrm{H}$ & $\mathrm{H}$ & $\mathrm{H}$ & $\mathrm{H}$ & $\mathrm{H}$ & $\mathrm{H}$ & $\mathrm{H}$ & H' \\
\hline $\mathrm{R}^{1}$ & $\mathrm{H}$ & $\mathrm{H}$ & $\mathrm{CH}_{3}$ & $\mathrm{H}$ & $\mathrm{H}$ & $\mathrm{H}$ & $\mathrm{H}$ & $\mathrm{H}$ & $\mathrm{H}$ \\
\hline $\mathrm{R}^{2}$ & $\mathrm{H}$ & $\mathrm{CH}_{3}$ & $\mathrm{CH}_{3}$ & $\mathrm{CH}_{3}$ & $\mathrm{CN}$ & $\mathrm{H}$ & $\mathrm{H}$ & $\mathrm{H}$ & $\mathrm{H}$ \\
\hline $\mathrm{R}^{3}$ & $\mathrm{H}$ & $\mathrm{H}$ & $\mathrm{H}$ & $\mathrm{CH}_{3}$ & $\mathrm{H}$ & $\mathrm{H}$ & $\mathrm{H}$ & $\mathrm{H}$ & $\mathrm{H}$ \\
\hline $\mathrm{R}^{4}$ & $\mathrm{H}$ & $\mathrm{H}$ & $\mathrm{H}$ & $\mathrm{H}$ & $\mathrm{H}$ & $\mathrm{OCH}_{3}$ & $\mathrm{H}$ & $\mathrm{H}$ & $\mathrm{H}$ \\
\hline $\mathrm{MtX}_{\mathrm{n}}^{-}$ & $\mathrm{SbF}_{6}$ & $\mathrm{SbF}_{6}=$ & $\mathrm{SbF}_{6}=$ & $\mathrm{SbF}_{6}=$ & $\mathrm{SbF}_{6}$ & $\mathrm{SbF}_{6}$ & $\mathrm{AsF}_{6}$ & $\mathrm{PF}_{6}$ & $\mathrm{BF}_{4}$ \\
\hline
\end{tabular}

A

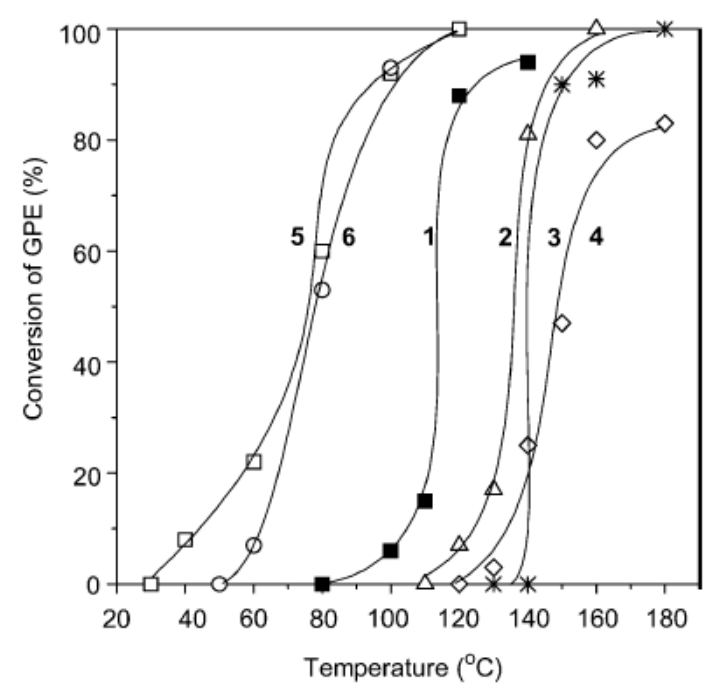

B

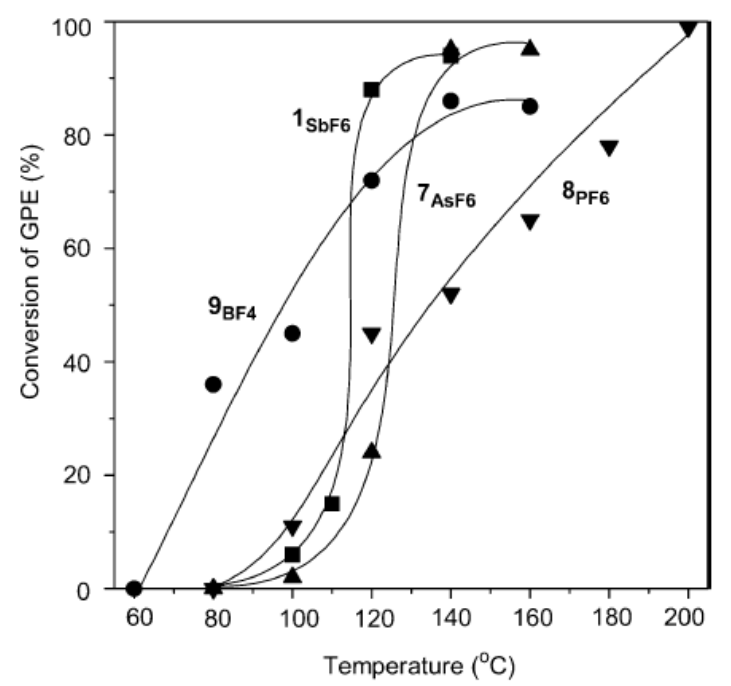

Figure 62 : (A) Temperature-conversion relationships in the bulk polymerization of GPE with the pyrazinium salts 1 to 6 (see Table 1) used at the molar ratio $3 \mathrm{~mol} \%$ after a period of 2 hours (C) Temperature-conversion relationships in the bulk polymerization of GPE with the pyrazinium salts 1 and 7 to 9 (see Table 1) used at the molar ratio 3 mol\% after a period of 2 hours (reproduced after (348) with permission from the American Chemical Society)

For instance, the activity of benzyl pyrazinium salts can be tailored by introducing either electron-donating or electron withdrawing substituent on the pyrazinium ring (Table 1). Figure $62 \mathrm{~A}$ illustrates the conversion profiles as a function of temperature for the cationic polymerization of the glycidyl phenyl ether (GPE) initiated by the benzylpyrazinium salts of hexafluoroantimonate with various substituents on the benzyl and pyrazinium rings. The results show that the introduction of electron-donating substituents $\left(\mathrm{CH}_{3}\right)$ on the pyrazinium ring is accompanied by an increase of the temperature threshold of initiation (entries 2, 3 and 4 of Table 1 and corresponding conversion profiles of Figure 62A). The opposite effect is 
observed when withdrawing substituents are introduced either on the benzyl or on the pyrazinium rings (entries 5 and 6 of Table 1 and corresponding conversion profiles of Figure 62A). The initiator activity is also dependent on the counteranion's nucleophilicity (entries 7, 8 and 9 of Table 1). Figure 62B represents the conversion profiles as a function of temperature for the cationic polymerization of GPE initiated by benzylpyrazinium salts with various counteranions. Clearly, as the nucleophilicity of the counteranion is decreased $\left(\mathrm{BF}_{4}^{-}>\right.$ $\mathrm{PF}_{6}^{-}>\mathrm{AsF}_{6}^{-}>\mathrm{SbF}_{6}^{-}$) the sharpness of the thermal response of the initiator is improved. The same type of study was carried out with the other main onium salt families, including sulfonium [331], anilinium [345] and phosphonium [67, 340]. This strategy has allowed the design of effective latent catalyst with threshold temperatures ranging from $60^{\circ} \mathrm{C}$ to $200^{\circ} \mathrm{C}$. Most of reported examples are distributed in the upper part of this temperature range (e.g. above $\left.100^{\circ} \mathrm{C}\right)$.

Concurrently, Yagci and coworkers proposed another approach to control the thermal initiation of cationic polymerization. Similarly to photolatent onium initiators that may be activated through the use of free radical coinitiator upon visible light, they suggested to activate thermolatent cationic initiators by using free radical sources that are released at lower temperature than the normal onium salt's temperature threshold [349-352]. To this end, they developed a series of specially designed allyl onium salts. Their typical chemical formulas are depicted in Figure 63. They include allyl sulfonium, allyl ammonium and allyl phosphonium salts. The mechanism of the free radical promoted initiation is illustrated in Figure 64 for the system based on the combination of an allyl pyridinium salt of hexafluoroantimonate with 2,2'-azobis(isobutyronitrile) (AIBN) as a radical coinitiator. The addition of the thermally generated radical on the allylic moiety of the onium salt results in the formation of a radical cation that subsequently undergoes a rapid fragmentation to release a new radical cation capable to initiate cationic polymerization. Using the same radical coinitiator, Yagci and coworkers demonstrated that the cationic polymerization of cyclohexene oxide initiated by allyloxypyridinium salts may be fully completed within 1 hour at $70^{\circ} \mathrm{C}$. In the absence of the coinitiator, the conversion is smaller than $10 \%$ after 1 hour at the same temperature. The conversion profiles of the two systems are compared in Figure 65. Allylsulfonium salts [353], allylpyridinium salts [354], allyloxypyridinium salts [355-357], allylanilinium [358] salts and allylphosphonium salts [295] were synthesized and successfully used in combination with thermal radical initiators to polymerize a variety of epoxy monomers. The numerous radical 
initiators, including AIBN or benzoyl peroxide (BPO), offer flexibility to this initiation strategy.

However, it is important to note that the use of radical intermediates results in the loss of the main advantage of cationic polymerization over its radical counterpart: the insensitivity towards oxygen.

In this context and under the pressure of their "greener" photolatent cousins, thermolatent onium initiators have progressively loss momentum over the past few years. To the best of our knowledge, the last new thermal latent onium salts were reported by Gupta and coworkers in 2009 [359-361]. The future of this research field will be dependent on the development of molecular structures with highly effective latent properties in very moderate temperature ranges.

From the viewpoint of practical and industrial applications, their use is now well established in a number of technical domains including molding and coating.<smiles>[R]C(=C)C[S+]1CCCC1</smiles>

Allyl sulfonium<smiles></smiles>

Allyl pyridinium<smiles>[R]C(=C)CO[N+]12C=CC(=P1)C=P2</smiles>

Allyl alkoxypyridinium

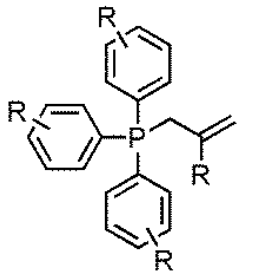

Allyl phosphonium

Figure 63: Structures of common allyl onium salts used in thermally induced radical fragmentation of onium salts to initiate cationic polymerization of epoxides.



Figure 64: General mechanism for the thermally induced radical fragmentation of onium salts illustrated with the example of an allyl pyridinium salt.

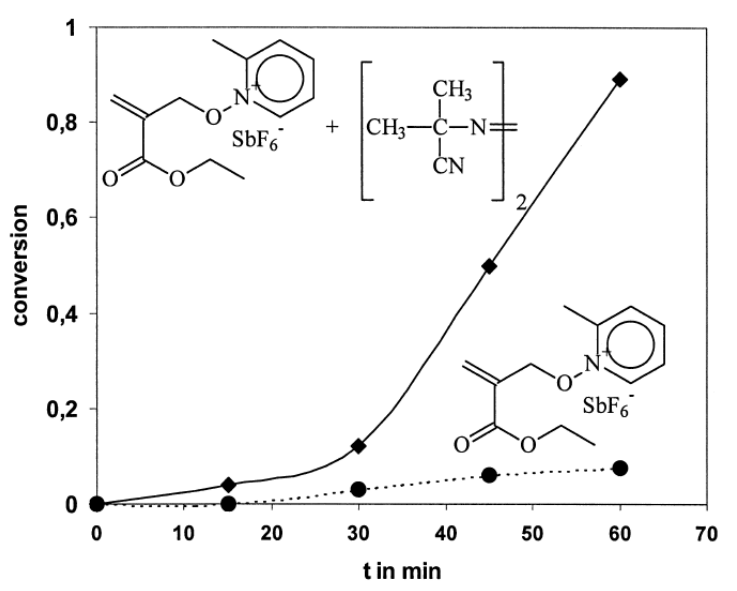

Figure 65: Conversion profiles for the thermal polymerization of cyclohexene oxide initiated by an allyl alkoxypyridinium salt $\left(\mathrm{c}=5.10^{-3} \mathrm{~mol} . \mathrm{L}^{-1}\right)$ alone or in combination with a thermal radical initiator (AIBN, $c=5 \cdot 10^{-3}$ mol. $\mathrm{L}^{-1}$ ). Reaction is performed at $70^{\circ} \mathrm{C}$, the typical decomposition temperature of AIBN. The onium salt is normally stable at this temperature (reproduced after ${ }^{(35 I)}$ with permission from Wiley) 


\subsubsection{Supramolecular control of the initiation}

From above-mentioned examples, it appears that, in most of the cases, cationic polymerization is actually initiated by a strong Brønsted acid $\mathrm{HMtF}_{\mathrm{n}}$ where $\mathrm{Mt}$ is a metalloid. Classically, Mt = B (n=4), P (n=6), As $(n=6)$ or Sb (n=6) [362, 363]. Once the Brønsted acid is released, either by photolysis or thermolysis of an onium salt but also by the thermolysis or hydrolysis of a $\mathrm{BF}_{3}$-amine complex (see section 3.3.1.2), epoxy monomers are rapidly activated and polymerization proceeds very quickly according to the ring opening polymerization mechanism (Figure 66A).

However, prior to the growth of the polymer chains, the activation of the monomers is in competition with the acid/base equilibria resulting from the presence of the various basic chemicals that may be contained in the resin [364]. Typically, this could be residual water whose reaction with the Brønsted acid $\mathrm{HMtF}_{\mathrm{n}}$ results in the formation of an hydronium ion, $\mathrm{H}_{3} \mathrm{O}^{+}[108,283]$. Under this form, the Brønsted acid is still involved in a rapid exchange between all the basic species present in the medium, including the monomers, and the onset of polymerization is rapidly observed as depicted in Figure 66B.

A

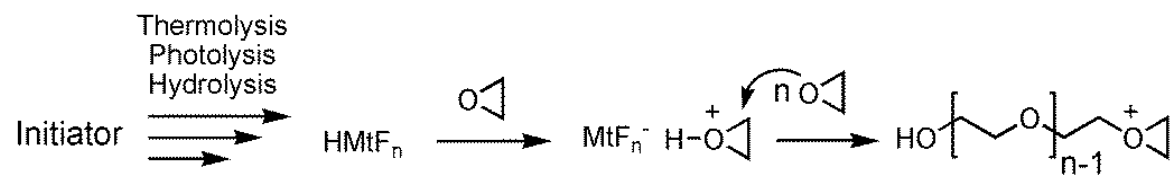

B

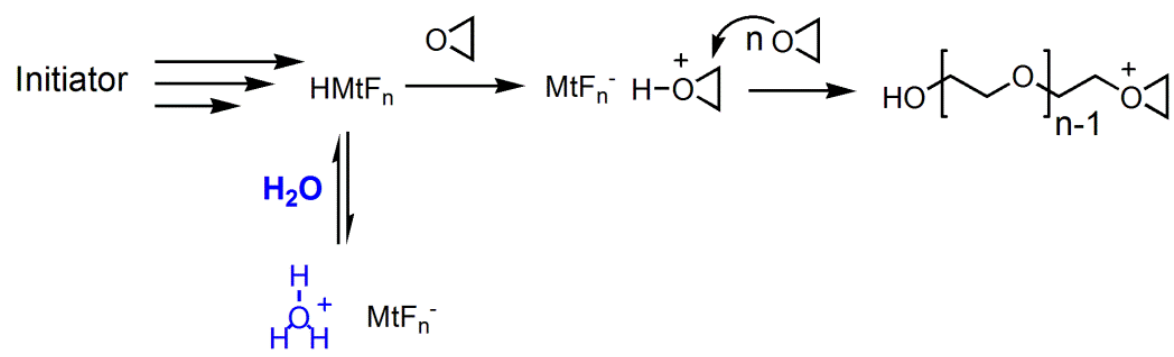

C

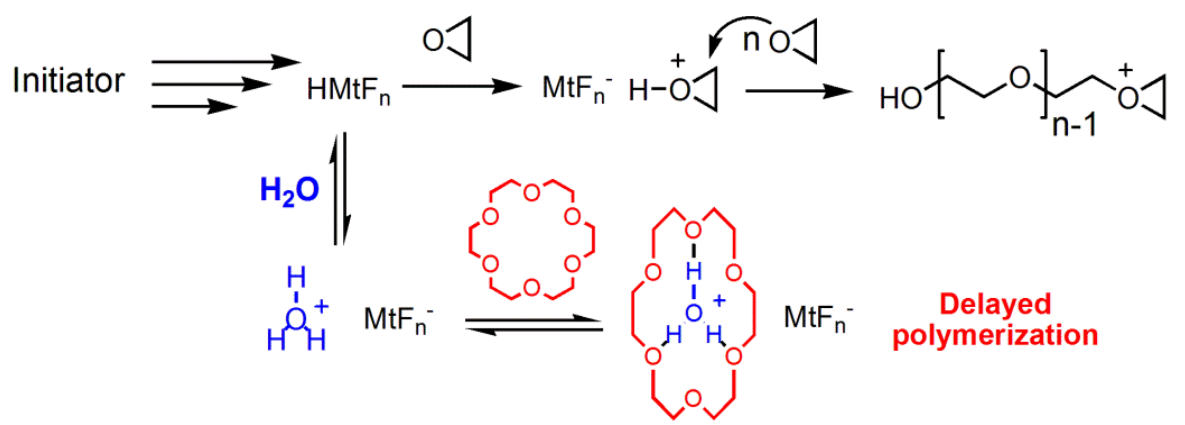

Figure 66: Initiation mechanism with the formation of a strong Brønsted acid $\mathrm{HMtF}_{\mathrm{n}}$ where $\mathrm{Mt}$ is a metalloid (classically $\mathrm{Mt}=\mathrm{B}, \mathrm{P}, \mathrm{As}$ or $\mathrm{Sb}$ ) and $n$ is the number of fluorine atom linked to the metalloid center ( $n=4$ for $\mathrm{B}, 6$ for $\mathrm{P}$, As and $\mathrm{Sb}$ ). (A) Initiation when the only basic species are the epoxy monomers (B) Initiation in the presence of water (C) Initiation in the presence of water and a crown ether, 18-crown-6. 
Crivello et al. reported the possibility to stabilize the Brønsted acid in the form of an hydronium ion through the complexation of $\mathrm{H}_{3} \mathrm{O}^{+}$by a crown ether, 18-crown-6 [283], whose cavity size and symmetry perfectly fit the $\mathrm{C}_{3 \mathrm{v}}$ geometry of $\mathrm{H}_{3} \mathrm{O}^{+}$(Figure 66C) [365]. Using this strategy, they observed retardation periods of the order of seconds to minutes for the photopolymerization of epoxy resins initiated by various onium salts. This approach provides additional flexibility to the control of photopolymerization but it is very dependent on the amount of water present in the resin.

Still aiming at releasing a strong Brønsted acid in a well controlled manner, Vidil et al. proposed the concept of supramolecular initiator [366]. The underlying idea of this approach is to shield the ammonium moiety of the tetrafluoroborate salt, $\mathrm{BF}_{4}{ }^{-}-\mathrm{RNH}_{3}{ }^{+}$, that acts as the true initiator in standard $\mathrm{BF}_{3}$-amine cured epoxy resins and to be able to deprotect it and initiate the cationic polymerization for low curing temperatures. Interestingly, in this system, the sequestration of the Brønsted acid is no longer dependent on the amount of residual water present in the resin.

In order to design such a system, Vidil et al. selected a low pKa amine, namely the 4chloroaniline $\left(4 \mathrm{CANH}_{2}, \mathrm{pKa} \cong 4.1\right)$. Under its deprotected form, the corresponding tetrafluoroborate ammonium salt, $\mathrm{BF}_{4}{ }^{-}-4 \mathrm{CANH}_{3}{ }^{+}$, readily release the strong Brønsted acid, $\mathrm{HBF}_{4}$ and polymerization can be initiate at room temperature [63].

On the basis of many published structures of primary ammonium and crown ether complexes [367-371], 18-crown-6 was selected as the hosting molecule to shield the ammonium moiety of $\mathrm{BF}_{4}{ }^{-}-4 \mathrm{CANH}_{3}{ }^{+}$. Indeed, similarly to $\mathrm{H}_{3} \mathrm{O}^{+}, \mathrm{NH}_{3}{ }^{+}$, exhibit the right symmetry $\left(\mathrm{C}_{3 \mathrm{v}}\right)$ and an appropriate ionic radius $(4.9 \AA)$ to fit the inner cavity of 18 -crown-6 and form a stable supramolecular complex. It is stabilized by the formation of three hydrogen bonds. Under its protected form, the dissociation rate of $\mathrm{BF}_{4}{ }^{-}-4 \mathrm{CANH}_{3}{ }^{+}$into the corresponding Brønsted acid $\left(\mathrm{HBF}_{4}\right)$ and primary amine $\left(4 \mathrm{CANH}_{2}\right)$ is considerably reduced. Moreover, the ammonium moiety is effectively protected from the direct nucleophilic attacks of epoxy monomers. A slight increase of temperature can disrupt the labile hydrogen bonds and results in the release of the highly reactive tetrafluoroborate ammonium salt, $\mathrm{BF}_{4}{ }^{-}-4 \mathrm{CANH}_{3}{ }^{+}$, as depicted in Figure 67. 
Overall, this system is expected to provide a supramolecular initiator that is stable and inactive at room temperature and that is able to release a strong Brønsted acid for moderate curing temperature.

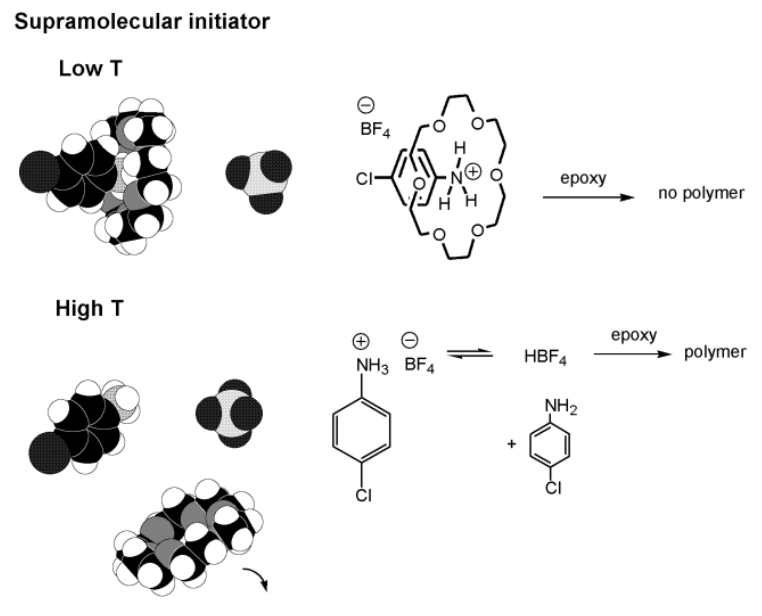

Figure 67: Schematic representation of the supramolecular initiation principle proposed by Vidil et al. An ammonium tetrafluoroborate-crown-ether is used as a supramolecular initiator. As temperature increases, the complex dissociates with release of a Brønsted acid, $\mathrm{HBF}_{4}$, which is able to trigger the cationic ROP of an epoxy monomer (reproduced after (366) with permission from the Royal Society of Chemistry).

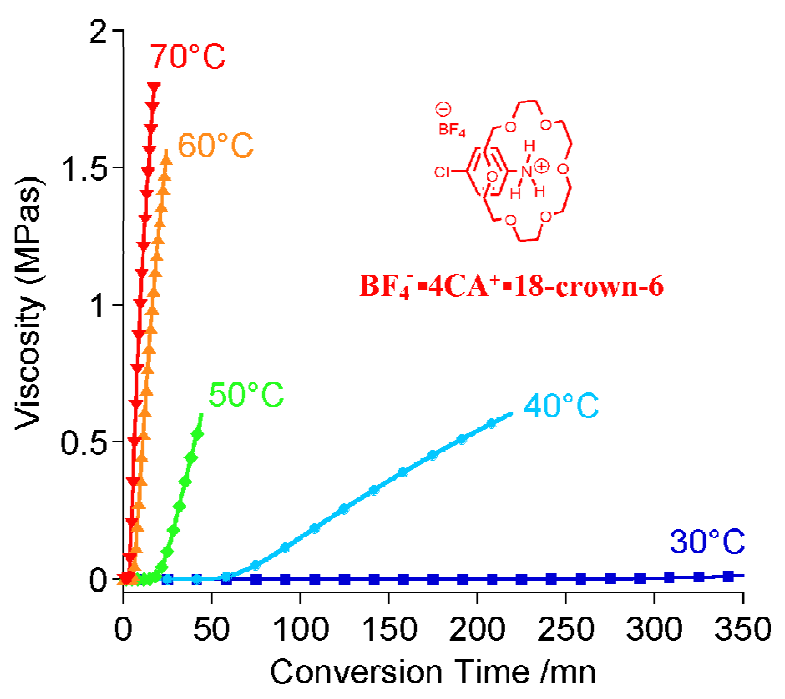

Figure 68: Time dependence of viscosity (measured by rheometry in the oscillatory mode at $1 \mathrm{~s}^{-1}$ ) during curing of DGEBA initiated by 0.026 eq. per epoxy of $\mathrm{BF}_{4}-4 \mathrm{CA}^{+} \cdot 18$ crown-6 at various temperature (reproduced after (366) with permission from the Royal Society of Chemistry).

Vidil et al. successfully isolated the supramolecular complex $\mathrm{BF}_{4}{ }^{-}-4 \mathrm{CANH}_{3}{ }^{+} \bullet 18$-crown- 6 and demonstrated that it could be used as a thermoresponsive initiator. Figure 68 presents the viscosity profile for the curing of DGEBA initiated by $\mathrm{BF}_{4}{ }^{-}-4 \mathrm{CANH}_{3}{ }^{+} \bullet 18$-crown- 6 at various temperatures. The profile remains unchanged after hours at room temperature while a sharp increase of viscosity is observed after a few minutes at $70^{\circ} \mathrm{C}$. The quantitative measurement of the gel points show that a gel is obtained in five minutes at $70^{\circ} \mathrm{C}$ and 1 day at $20^{\circ} \mathrm{C}$. Moreover, the activation energy, $E_{a}$, were estimated from the temperature dependence of the gel point for the curing of DGEBA initiated by the supramolecular initiator $\mathrm{BF}_{4}^{-}$ $-4 \mathrm{CANH}_{3}{ }^{+} \bullet 18$-crown-6 as well as the unprotected salt $\mathrm{BF}_{4}{ }^{-}-4 \mathrm{CANH}_{3}{ }^{+}$, and the corresponding $\mathrm{BF}_{3}$-amine complex, $\mathrm{BF}_{3}-4 \mathrm{CANH}_{2} . E_{a}$ is close to $70 \mathrm{~kJ} / \mathrm{mol}$ for both $\mathrm{BF}_{3}-4 \mathrm{CANH}_{2}$ and $\mathrm{BF}_{4}{ }^{-}-4 \mathrm{CANH}_{3}{ }^{+}$, confirming that they share the same initiation mechanism (see section 3.3.1.2). A significantly higher value of $100 \mathrm{~kJ} / \mathrm{mol}$ is measured for the supramolecular initiator $\mathrm{BF}_{4}{ }^{-}-4 \mathrm{CANH}_{3}{ }^{+} \bullet 18$-crown-6. As mentioned in section 3.3.2.2, $E_{a}$ can be used to rank initiators according to their thermal stability. Here, the increase of activation 
energy of about $30 \mathrm{~kJ} / \mathrm{mol}$ in the case of the supramolecular initiator is a rough quantification of the gain of stability conferred by the crown ether to the active species $\mathrm{BF}_{4}{ }^{-}-4 \mathrm{CANH}_{3}{ }^{+}$.

When compared to classical thermolatent onium salts (see section 3.3.2.2.), the activation energy of the supramolecular initiator is of similar magnitude $(100 \mathrm{~kJ} / \mathrm{mol})$ but it initiates the cationic polymerization in a much lower range of temperatures. For instance, sulfonium salts [327] are usually inactive for temperatures below $100^{\circ} \mathrm{C}$. On the other hand, the supramolecular initiator exhibits the right combination of stability and thermal responsivity to provide long pot-life at ambient temperature and fast curing rates as temperature rises below $100^{\circ} \mathrm{C}$. These are appropriate characteristic features to minimize the energy consumption of the curing system but also to reduce the extent of monomer degradation, side reactions as well as VOC emission.

In the end, the supramolecular complexation of Brønsted acids is a promising method to control the polymerization onset. It is a flexible approach, based on the disruption of weak physical bonds that provides long pot-life while preserving the possibility to trigger the polymerization in a reasonable temperature range. On the opposite side, systems based on the cleavage of strong covalent bonds cannot be activated in the same temperature range.

It is noteworthy that Crivello and Aldersley reported the complexation of another cationic species, namely the iodonium cation of diaryl iodonium photoinitiators [372]. The structure of the complex resulting from the interaction of a diaryl iodonium salt and 18-crown-6 determined by X-ray diffraction is shown in Figure 69. This complexation results in a substantial increase of the storage lifetimes of monomer's mixtures at elevated temperature. Again, this bodes well for application of supramolecular complexation to control the thermal polymerization of epoxy thermosets.
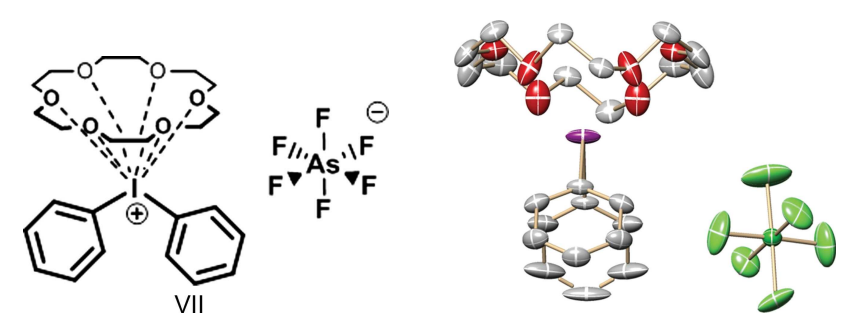

Figure 69: Chemical structure of the complex of a diaryliodonium initiator and a crown ether as reported by Crivello and Aldersley (reproduced after (372) with permission from Wiley). 


\section{Control of the chain growth during the crosslinking process}

The possibility to delay the occurrence of the gel point by controlling the value of the critical conversion, $x_{\text {gel }}$, was briefly discussed in section 2.3.3. Differences between step and chain ring-opening polymerizations were emphasized and the possibility to tailor the gelation in the case of the ring-opening polymerization by using chain transfer agents (CTAs) was mentioned. A schematic representation of the effect of CTA on the crosslinking process is depicted in Figure 70. In the absence of CTAs (Figure 70A), the polymer chains grow linearly and are weakly branched so that gelation occurs in the early stages of polymerization. In contrast, in the presence of CTAs (Figure 70B), transfers promote branching and growth of many short chains. Gelation is observed for longer times and higher monomer conversions. In this section, an overview of the typical CTAs used in anionic and cationic chain polymerizations of epoxy is proposed. The main results reported in the literature regarding the use of this strategy in thermoset technology are described. Special attention is paid to cationic polymerization.
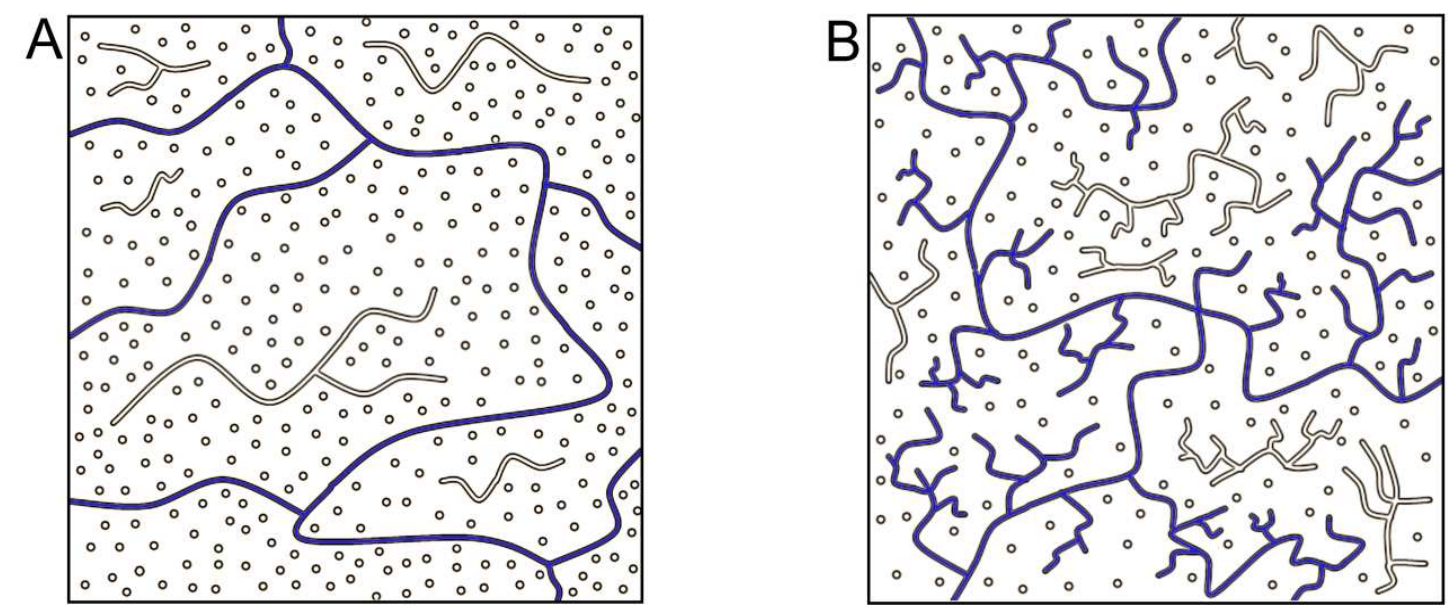

Figure 70: Schematic representation of a growing network at the gel point in the presence (A) and in the absence (B) of CTAs. Free unreacted monomers are depicted as open circles, whereas the chains involved in the gel macromolecule are represented in blue (reproduced after (373) with permission from the American Chemical Society)

\subsection{Anionic homopolymerization}

Protic substances such as water or alcohol are the main CTAs considered in ring opening anionic polymerization of epoxy. In the field of thermosetting chemistry, they can be present in commercial resins in trace amounts but they may also be added to the curing mixture for various purposes (such as shrinkage or toughness control agents). Their presence results in the transfer of alkoxide active moieties between propagation chains and alcohols as depicted in Figure 71A. In a first approach, these transfers are equivalent to classical chain-transfer 
reactions. However, they result in the formation of polymeric alcohols that are not dead but simply dormant. For this reason, these chain transfers are often called exchange reactions [98].

A)

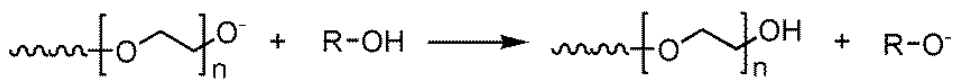

B)

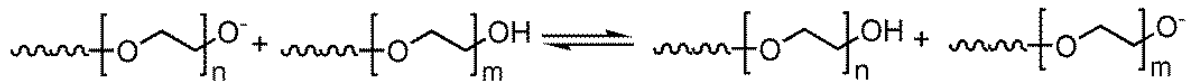

Figure 71: Exchange reaction in anionic polymerization of epoxides. (A) Exchange reaction between a propagation chain and an alcohol. (B) Exchange reaction between the newly formed polymeric alcohol and other propagating chains (remark: for simplicity's sake, the alkoxide's counterion is not represented).

Indeed, all alcohol and alkoxide molecules in the reaction system are in dynamic equilibrium. Each polymer chain alternates between the active propagating alkoxide and the dormant alcohol forms. The situation is illustrated in Figure 71B. These exchange reactions lower the polymer's molecular weight and the number-average degree of polymerization can be estimated by Eq. (6).

$$
\bar{X}_{n}=\frac{[M]_{0}-[M]_{t}}{[I]+[R O H]}
$$

$[M]_{0}$ and $[M]_{t}$ are the monomer concentrations at times zero and $t,[\Pi]$ is the initiator concentration and $[\mathrm{ROH}]$ the alcohol concentration. This formula illustrates that each alcohol molecule contributes equally with an initiator species to determining the number of propagating chains. One can estimate the amount of added alcohol required to achieve some desired value of the number average degree of polymerization and thus of the conversion at the gel point according to Eq. (3) (see section 2.3.3.2).

As a result, for a crosslinking process, critical conversion at the gel point considerably increases in the presence of hydroxyl groups and the occurrence of gelation is delayed to the same extent. Early works were performed by Rozenberg and coworkers and are reported in a detailed review [48]. Recently, in a series of papers, Serra and coworkers described studies of the curing of DGEBA initiated by 1-methylimidazole (see section 3.2.1) in the presence of various hydroxyl-ended multiarm polymers [114, 374-377]. For instance, they used a star polymer based on a poly(styrene) core and poly( $\varepsilon$-caprolactone) arms as a reactive modifier to improve the toughness of final materials [374]. The structure of this additive is depicted in Figure 72. When the star modifier was added to the formulation, they observed an increase of 
both the gel time and the critical conversion. The critical conversion was doubled from $34 \%$ to $68 \%$. They showed that this large increase could be beneficial from the processing standpoint by reducing the formation of internal stress as curing proceeds. The same group also reported the influence of hydroxyl moieties on the anionic chain copolymerization of DGEBA (oligomeric DGEBA, with different $n$ numbers, see Figure 1, section 2.1.1) with bislactone [62,378] or anhydride [379] comonomers. As in the case of homopolymerization, it was observed that an increase of the concentration of $\mathrm{OH}$ group is accompanied by an increase of the conversion at the gel point. Reported values of $\mathrm{x}_{\mathrm{gel}}$ range from 22 to $48 \%$ depending on the $\mathrm{OH}$ content. Following these works, Fernàndez-Francos proposed a theoretical modeling of the effect of proton donors in the network build-up of epoxy thermosets using tertiary amines as initiators [121]. This recent study provides a flexible model with the potential to describe the curing behavior of different epoxy formulations from homo- to copolymerization. Figure 73 illustrates the theoretical variation of the critical conversion according to Fernandez-Francos' model for the anionic polymerization of DGEBA as a function of the initiator concentration in the absence or in the presence of transfers. As expected, formulations with added monoalcohols exhibit higher conversion at the gel point than the formulation free of CTAs whatever the molar fraction of initiator one considers.

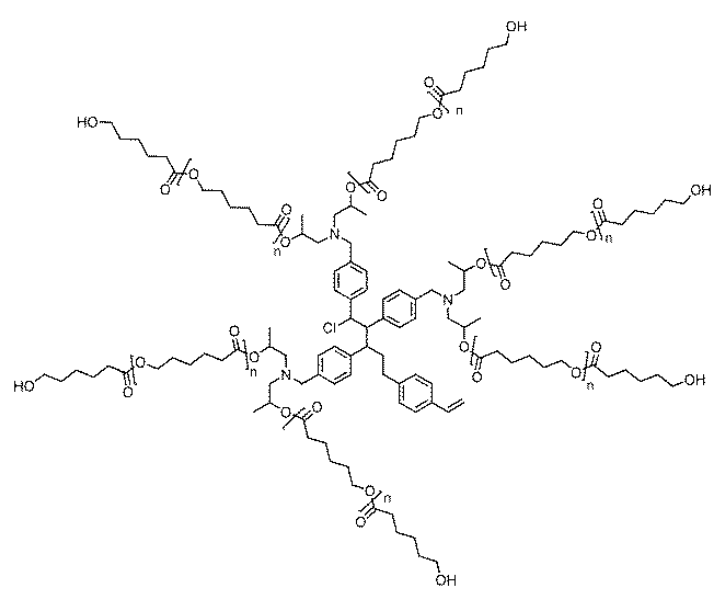

Figure 72: The multiarm star polymer (poly(styrene) core and poly( $\varepsilon$-caprolactone) arms) used as a reactive modifier by Serra et al. in the anionic polymerization of DGEBA initiated by 1-methyl imidazole. The modifier is covalently incorporated in the network thanks to exchange reactions.

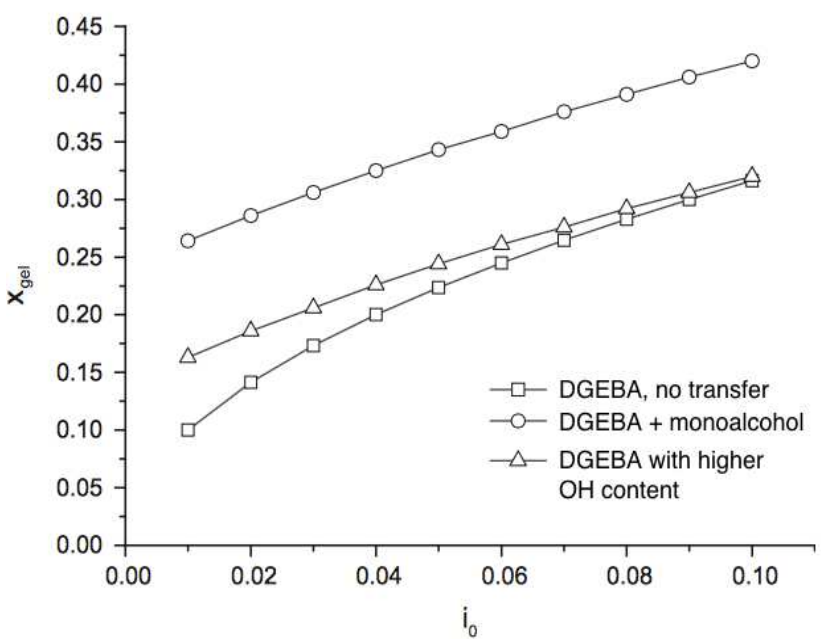

Figure 71: Theoretical variation of the critical conversion, $x_{g e l}$, for the anionic polymerization of DGEBA in the presence of alcohol chain transfer agents as a function of the initiator mol fraction, $\mathrm{i}_{0}$, according to Fernandez-Francos' model. Alcohols are considered as added monoalcohol (empty circles) or as substituents of oligomeric DGEBA with higher molecular weight (i.e. higher $\mathrm{OH}$ content, empty triangle) (reproduced after ${ }^{(121)}$ with permission from Elsevier). 


\subsection{Cationic homopolymerization}

\subsubsection{Chain transfer and Activated Monomer (AM) mechanism}

As depicted in Figure 74A, cationic ring-opening polymerization of epoxides can undergo transfer reactions through nucleophilic attacks of the oxonium ion propagating center. Among potential nucleophilic groups, etheral groups present in the growing chain may compete with the cyclic monomers for the attack of the propagating center (see section 2.1.3.2 for a reminder of the cationic polymerization mechanism). A schematic illustration of this mechanism, named back-biting is proposed in Figure 74B. It results in the formation of cyclic oligomers. The formation of 1,4-dioxane is extremely favored due to the high stability of this size of ring. For polymerization of the simplest epoxy monomer, ethylene oxide, 1,4-dioxane can be formed in yield as high as $80 \%$ due to the absence of any steric hindrance on the growing chains [380]. Thus, in some extreme circumstances, back-biting may completely preclude the polymerization.

A)

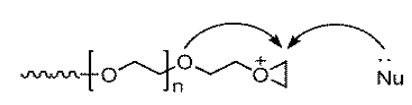

B)

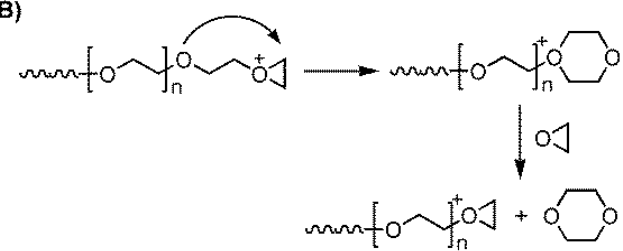

C)

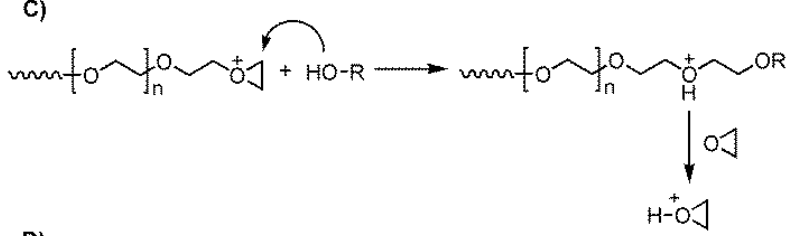

D)

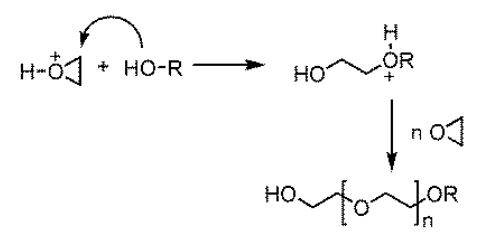

Figure 74: Typical chain transfers involved in cationic polymerization of epoxy. (A) Intramolecular and intermolecular attack of a chain end propagating center. (B) Intramolecular attack of the propagating center and formation of 1,4-dioxane. (C) Attack of a chain end propagating center by a monoalcohol and formation of a dead end. (D) Attack of an activated monomer and formation of an alcohol extended by one monomeric unit. Successive attacks of activated monomer result in the growth of a polymer chain.

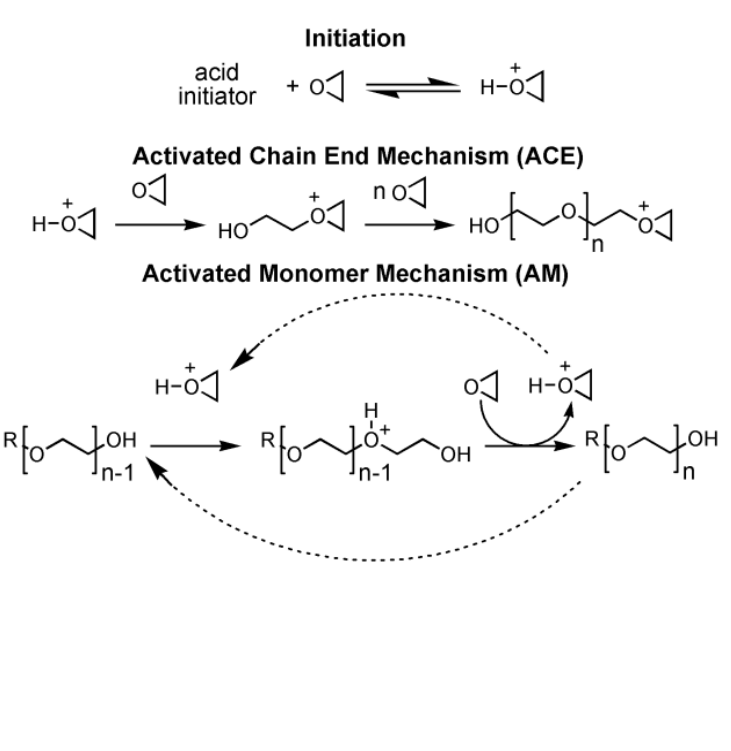

Figure 72: The two mechanisms of the ring opening cationic polymerization of epoxy in the presence of alcohol: the AM (Activated Monomer) and the ACE (Activated Chain End) mechanisms (reproduced after (373) with permission from the American chemical Society). 
Apart from etheral groups that promote intramolecular transfers, the most common transfer agents encountered in cationic polymerization are hydroxyl groups. Hydroxyl groups may attack both (i) propagating centers (tertiary oxonium ion, Figure 74C) and (ii) activated monomers (secondary oxonium ion, Figure 74D).

(i) In the former case, if the alcohol is monofunctional, this results in a true transfer reaction with the formation of a dead end. If the alcohol is polyfunctional, then a macroalcohol is obtained which may be involved in another transfer reaction or in the mechanism presented in (ii).

(ii) In the latter case, the addition of the alcohol to an activated monomer results in the formation of a new hydroxyl-terminated molecule extended by one monomeric unit. Thus, each time an $\mathrm{OH}$ group is consumed another $\mathrm{OH}$ group is produced. In this condition, the process is not called a chain transfer anymore. Instead, it could be seen as an effective polymerization mechanism proceeding through successive addition of activated monomers to macromolecular alcohols' end.

Penczek and coworkers first described this polymerization mode [125]. It was called an Activated Monomer (AM) mechanism in opposition to the classical mechanism for which the term Activated Chain End (ACE) mechanism is used [381]. Thus, for the cationic polymerization of epoxides (and by extension for all cyclic ethers), two propagation mechanisms are expected in the presence of alcohols. The situation is summarized in Figure 75. The remarkable characteristic feature of the AM mechanism is that it proceeds without a tertiary oxonium ion at the end of the growing chain. Back-biting is prevented and there is no way to produce 1,4-dioxane. Thus, in order to suppress formation of cycles one has to eliminate or at least to decrease the relative contribution of the ACE mechanism in favor of the AM propagation route. The rate of the ACE mechanism is proportional to the concentration of monomer and of activated monomer. It is given by Eq. (7). Concurrently, the rate of the AM mechanism is proportional to the concentrations of alcohol and of activated monomer as indicated by Eq. (8). In this condition, the relative rate of the AM mechanism, $R_{A M} /\left(R_{A M}+R_{A C E}\right)$, is given by Eq. (9) which, after simplification, yields Eq. (10) where $R_{A M} /$ $/\left(R_{A M}+R_{A C E}\right)$ appears as an increasing function of the alcohol to monomer ratio. Since the alcohol concentration is constant, Penczek et al. concluded that one has to decrease the instantaneous monomer concentration to prevent the ACE route, i.e to observe 
$\boldsymbol{R}_{A M} /\left(\boldsymbol{R}_{A M}+R_{A C E}\right) \rightarrow \mathbf{1}$. To this end, monomers can be added to an initiator and alcohol mixture in a continuous manner at a rate equal to its rate of consumption. By doing so, the group of Penczek reported several examples where they considerably reduce the formation of cyclic oligomers [382-384]. This new synthesis strategy significantly contributed to the popularization of the AM mechanism.

$$
\begin{aligned}
& \left.\left.R_{A C E}=k_{A C E}\left[\mathrm{H}^{+} \mathrm{O}\right]\right][0\}\right] \\
& \left.R_{A M}=k_{A M}\left[\mathrm{H}^{+} \mathrm{O}\right]\right][\mathrm{ROH}] \\
& \frac{R_{A M}}{R_{A M}+R_{A C E}}=\frac{\left.k_{A M}\left[\mathrm{H}^{+} \mathrm{O}\right]\right][\mathrm{ROH}]}{\left.k_{A M}\left[\mathrm{H}^{+} \mathrm{O} J\right][\mathrm{ROH}]+k_{A C E}\left[\mathrm{H}^{+} \mathrm{O}\right]\right][\mathrm{OJ}]} \quad \text { Eq. (9) } \\
& \frac{R_{A M}}{R_{A M}+R_{A C E}}=\frac{k_{A M}[\mathrm{ROH}]}{k_{A M}[\mathrm{ROH}]+k_{A C E}[\mathrm{OJ}]} \underset{[\circ] \rightarrow 0}{\longrightarrow} 1
\end{aligned}
$$

\subsubsection{Control of the critical conversion}

Following the seminal works of Penczek and coworkers, the notion of AM mechanism has been used to explain the curing behavior of three-dimensional epoxy networks in the presence of alcohols. In this one-pot condition (in opposition to the starved monomer conditions proposed by Penczek et al.), alcohols will both act as (1) chain transfer agents and (2) promoters of the AM mechanism:

(1) As depicted in Figure 76A, if one considers a monoalcohol, the nucleophilic attack of activated chain ends results in true transfers. This is accompanied by an increase of the number of growing chains and thus a decrease of their molecular weight. Therefore, the conversion at the gel point is increased.

(2) Concurrently, if alcohols attack activated monomers then hydroxyl terminated chains can grow through the AM mechanism. Thus, as illustrated in Figure 76B, each hydroxyl group may be seen as a possible initiator of a new growing chain. In other words, the higher $[\mathrm{OH}]$ is, 
the higher the number of growing chains is and the higher the conversion at the gel point is. In that case, the role of alcohols is similar to the role they play in anionic polymerization. Obviously, if $[\mathrm{OH}]>$ [nitiator] then the critical conversion will be higher than in the case of the neat system (Figure 76B, first arrow). Moreover, since alcohol and epoxy coexist, the activated monomers liberated by the AM mechanism may be attacked by epoxy monomers and result in the formation of new chains growing through the ACE mechanism (Figure 76B, second arrow). This way, the number of growing chains is further increased.

Thus, from the viewpoint of the formation of three-dimensional networks, alcohols represent a suitable tool to control the value of the critical conversion at the gel point by increasing the branching degree. Furthermore, as depicted in Figure 77, they are incorporated into the network and the usual safety problems related to volatile additives are prevented. It is also an additional lever to tune the properties of the network such as the crosslink density, the $T_{g}$ or the mechanical properties. Indeed, by playing with the nature of the alcohol (functionality, size and nature of the skeleton), a number of options can be explored to tune the properties of the native resin.

A)

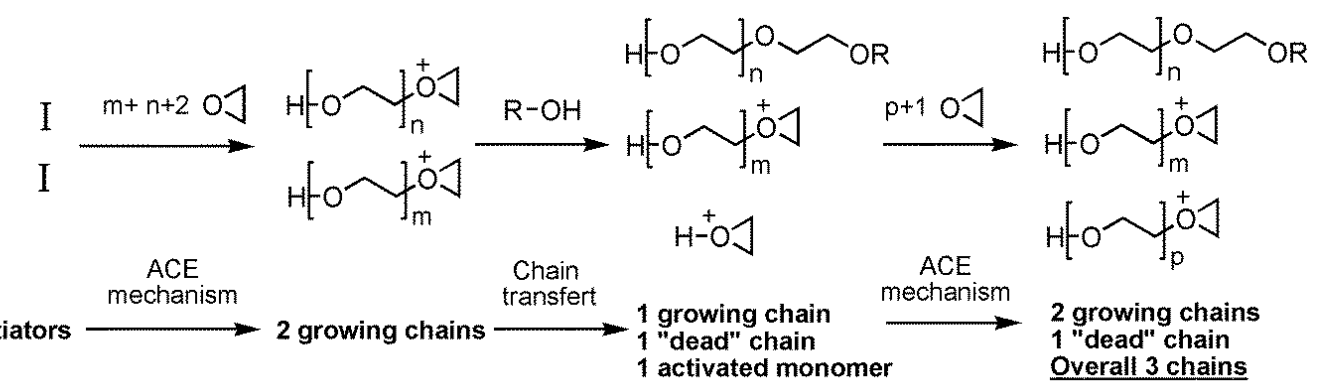

B)

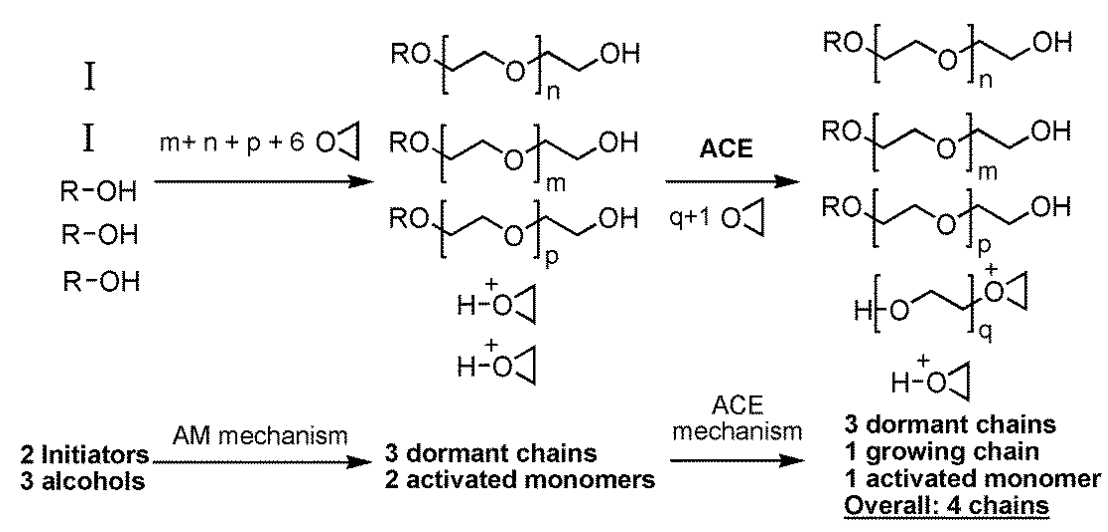

Figure 73: Illustration of various situations where alcohol are involved in chain transfers or the AM mechanism, resulting in the increase of the number of growing chains and thus of the branching degree of the growing network. (A) Illustration of the effect of chain transfer when starting from activated chains. (B) Illustration of the effect of an alcohol concentration larger than the initiator concentration and of the superposition of the ACE and AM mechanisms. 
Several authors studied the influence of alcohols in bulk polymerization of epoxy resins. Pascault and coworkers described the homopolymerization of DGEBA initiated by the $\mathrm{BF}_{3}$-amine complex of 4-chloroaniline. They used a short poly(ethylene oxide) (PEO $M_{n}=$ 300) to solubilize the initiator [262]. In these conditions, they observed high conversion at the gel point (40\%). It was attributed to the promotion of chain transfers and of the AM mechanism by the hydroxyl moieties of PEO. The resulting system is thought to be suitable for the reaction-injection-molding (RIM) process. Li and coworkers demonstrated that the cationic polymerization of oligomeric DGEBA initiated by $\mathrm{BF}_{3}$-ethylamine was greatly influenced by the hydroxyl content of the oligomeric resin [385]. Their results show that the evolution of the molecular weight of the polymer chains during the curing reaction is much slower in the case of a hydroxyl rich oligomer due to the chain transfers resulting from the presence of alcohol. For instance, Figure 78 illustrates the evolution of the molecular weight for two oligomeric DGEBA: DER331 and DER332. DER331 contains more hydroxyl groups than DER332. As a result, the increase of the molecular weight is much slower for DER331.

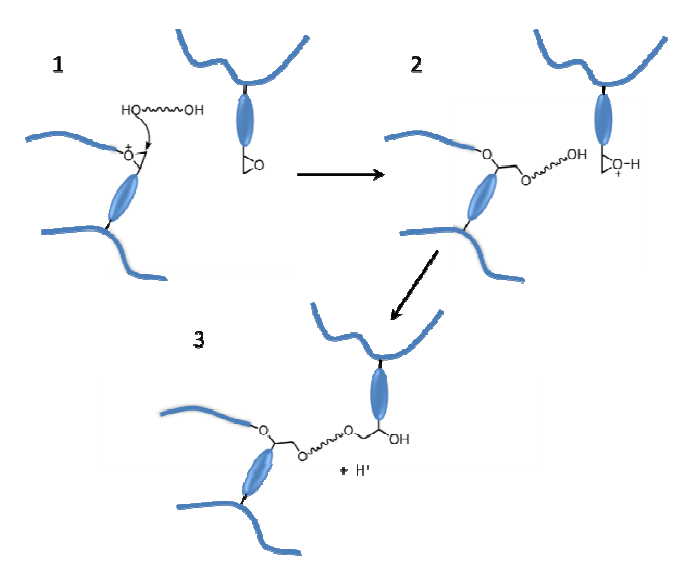

Figure 77: Schematic representation of one possible route resulting in the incorporation of a diol in a network growing through ring opening cationic polymerization.

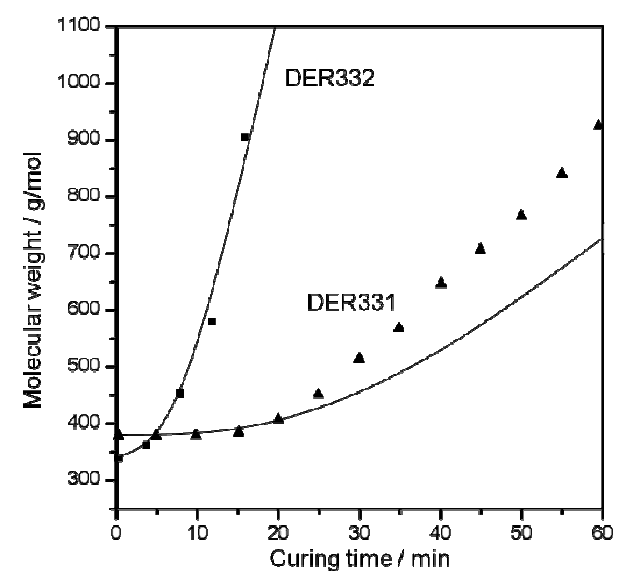

Figure 78: Molecular weight vs. reaction time for the cure of two

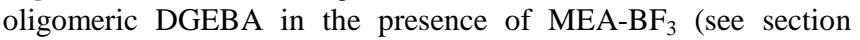
3.3.1) at $130^{\circ} \mathrm{C}$. The experimental results are fitted with a theoretical model proposed by $\mathrm{Li}$ et al. The temperature deviation is attributed to the experimental difficulty to control this parameter. DER 331 contains more hydroxyl groups than DER332 due to higher oligomerization degree (reproduced after (385) with permission from Wiley).

More recently, in a series of papers, Serra, Ramis and their coworkers described new thermosets obtained from the cationic curing of DGEBA in the presence of hydroxyl-ended hyperbranched polymers (HBP) [386-393]. The promotion of chain transfers and of the AM mechanism allows the covalent incorporation of the HBP into the network. It is accompanied by an expansion of the HBP resulting in the production of an excess of free volume that compensate for the contraction normally associated with the homopolymerization of epoxy. 
Concurrently, they observed a large increase of the critical conversion as the weight percentage of incorporated HBP is raised, a proof of the involvement of the alcohol moieties in the polymerization mechanism [390, 392, 393]. This last point represents an additional benefit as it contributes to the reduction of internal stresses developed during the curing process. Moreover, materials with improved flexibility were obtained by increasing the proportion of HBP and a progressive decrease of $T_{g}$ and storage modulus was observed [392]. In the same vein, Hartwig and coworkers conducted a series of works showing that the incorporation of polyols in cationically polymerized epoxy resins was an effective toughening method [394-399]. Interestingly, in that case, they reported that a partial inhibition of the AM mechanism may also be advantageous to limit the covalent incorporation of the polyols into the thermosetting matrix and promote phase separation by reaction induced phase separation (RIPS) [394]. Indeed, heterogeneous morphologies minimize the decrease of the $T_{g}$ and the storage moduli that is usually observed with classical toughening strategies. Figure 79 represents the scanning transmission electron microscopy (STEM) images of the morphology observed by Hartwig et al. for diepoxide cured in the presence of various poly( $\varepsilon$-caprolactone) (PCL). Figure 79A is the STEM image of the material cured with a PCL terminated with hydroxyl end group (PCL-diol), while Figure 79B is the STEM image of the equivalent resin cured with the same PCL whose hydroxyl end groups have been blocked by esterification (PCL-diester) so that the AM mechanism as well as potential covalent linkage with the epoxy network is prevented. Clearly, the prevention of the AM propagation route results in the phase separation of the PCL-diester with a sphere like nanostructure. Ramis and coworkers reported similar observations by using HBPs partially blocked with trimethylsilyl or benzoyl moieties [392].


Figure 74: STEM images of a cured diepoxide with the thermolatent initiator benzyl tetrahydrothiophenium hexafluoroantimonate in the presence of (A) 10wt\% of PCL-diol and (B) 10wt\% of PCL-diester. For the nanostructured material of image B, the domain size is $10-20 \mathrm{~nm}$. Scale bar for image (A) is $200 \mathrm{~nm}$ and for image (B) $100 \mathrm{~nm}$ (reproduced after (394) with permission from the Royal Society of Chemistry) 
Thus, incorporation of alcohols as promoters of the AM mechanism and transfer agents provides an effective lever to control the occurrence of the gel point by involving the initiation of many short chains and decreasing the average molecular weight. As an additional advantage, playing with the competition between the AM and the ACE mechanisms enables an adjustment of the morphological properties of the cationically polymerized epoxy resins and of their resulting thermo-mechanical properties.

\subsubsection{Control of the polymerization rate.}

From the stand point of the temporal-programming of the curing process, the competition between the $\mathrm{AM}$ and $\mathrm{ACE}$ mechanism is also an alternative to control the curing rate of thermoset materials.

Early work performed by Penczek and coworkers studied the polymerization's kinetics according to the two mechanisms $[383,400]$. They discussed the values of the initiation and propagation rate constants: $k_{i A M}, k_{i A C E}, k_{P A M}$ and $k_{P A C E}$ as reported in scheme of Figure 80 . In particular [400], they determined the ratio of $k_{i A M}$ and $k_{i A C E}$ in the systems where both alcohols and monomers are simultaneously present, and they found that, for epichlorohydrin, the ratio of rate constant was approximately 5:1, i.e:

$$
\frac{k_{i A M}}{k_{\text {iACE }}} \approx 5
$$

This result is in accordance with the greater nucleophilicity of hydroxyl groups toward protonated monomers in comparison with that of the oxirane ring oxygen atom.

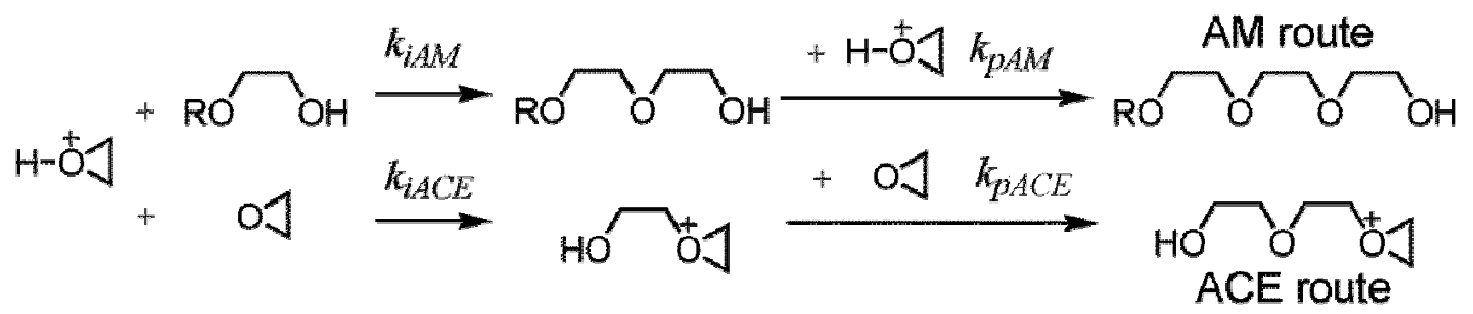

Figure 80: Schematic representation of the activated monomer (AM) and the activated chain end (ACE) mechanism routes for the cationic polymerization of epoxides. The initiation and propagation rate constants are reported.

As for the propagation rates, in the idealized case, the AM propagation mode can be reduced to one reaction and one can write:

$$
k_{i A M}=k_{p A M}
$$


Furthermore, by considering the respective nucleophilicity of the hydroxyl groups and the monomers once again, it comes:

$$
k_{i A M}>k_{p A C E}
$$

Thus, from Eq. (12) and Eq. (13), it is expected that:

$$
k_{p A M}>k_{p A C E} \quad \text { Eq. }(14)
$$

However, as first suggested by Penczek and coworkers [400], in the case of the AM mechanism, the proton released after each activated monomer addition can combine with the nucleophilic sites present in the curing system, including those distributed along the polymer chains (polyether patterns). Thus, the real set of elementary reactions involved in the AM mechanism is much more complicated and the rate constant of this propagation mode can be slower than expected. On the contrary, the ACE propagation mode relies on a single initiation step followed by consecutive addition of monomers to the growing chain end without recirculating protons. In these conditions, it is possible to observe propagation rates values ranked in reverse order compared to Eq. (14), i.e.:

$$
k_{p A M}<k_{p A C E} \quad \text { Eq. (15) }
$$

This was confirmed by Matejka and coworkers for the polymerization of DGEBA initiated by a $\mathrm{BF}_{3}$-amine complex. They determined the respective rate constants of propagation for the $\mathrm{AM}$ and the ACE mechanisms by comparing experimental and theoretical results $[69,119$, 120]. They found $k_{p A M}=0.12 \mathrm{~mol}^{-1} \cdot \mathrm{min}^{-1}$ and $k_{p A C E}=0.25 \mathrm{~mol}^{-1} \cdot \mathrm{min}^{-1}$. Thus, in this polymerization system, the promotion of the AM mechanism results in a decrease of the observed propagation rate, $\mathrm{R}_{\mathrm{p}}$.

This was also confirmed in several studies using $\mathrm{BF}_{3}$-amine complexes as the initiator of the curing reaction. Pascault, Chabanne and coworkers have shown that water and small hydroxyl byproducts (i.e. alcohols resulting from the opening of the first epoxy monomers), orient the propagation towards the $\mathrm{AM}$ route for the polymerization of PGE initiated by the $\mathrm{BF}_{3}-4-$ methoxyaniline complex [105]. The chemical structures of the hydroxyl byproducts they identified are depicted in Figure 81. These molecules can act as effective AM promoters. Figure 82 represents the conversion profiles obtained for the neat system and with addition of the hydroxylated compounds 1 and 2 (Figure 81). Clearly, the propagation rate is significantly decreased by increasing the amount of these impurities. Similarly, Pascault, Bouillon and 
coworkers reported a strong decrease of the polymerization rate for the curing of DGEBA initiated by the $\mathrm{BF}_{3}$-4-chloroaniline complex in the presence of a low molar mass poly(ethylene oxide) additive (PEO, $M_{n}=300 \mathrm{~g} \cdot \mathrm{mol}^{-1}$ ). By using PEO as a diluting agent of the $\mathrm{BF}_{3}$-amine initiator, they observed that the propagation rate, $\mathrm{R}_{\mathrm{p}}$, decreases with the PEO concentration, [PEO] $[63,261,262]$. They found a negative order of -0.55 with respect to [PEO] [261]. This was attributed to the promotion of the AM mechanism by the terminal hydroxyl groups of PEO. More recently, Vidil and coworkers investigated HO-terminated and alkyl-terminated PEO and confirmed that the presence of $\mathrm{OH}$ groups result in a decrease of the polymerization rate [373].<smiles>OCC(O)COc1ccccc1</smiles>

1<smiles>COc1ccc(N(CC(O)COc2ccccc2)CC(O)COc2ccccc2)cc1</smiles>

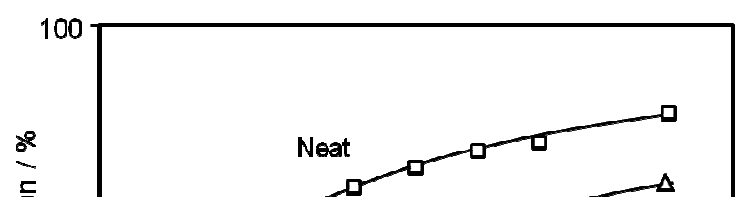


However, it is worth noting that, in the case of polymerization initiated with onium salts, several studies report an acceleration of the polymerization rate in the presence of alcohols. This was first reported by Crivello and coworkers for the photochemically induced polymerization of 3,4-epoxycyclohexylmethyl-3',4'-epoxycyclohexane carboxylate [402]. They observed an increase of the curing rate in the presence of ethylene glycol or 1,4butanediol. Later, they reported the synthesis of epoxy monomers that contain both epoxy and hydroxyl groups in the same molecule [285]. Again, the photopolymerization of these monomers initiated by diaryliodonium salt was markedly accelerated in comparison to their alcohol-free counterparts. Ramis, Serra and their coworkers made similar observations when incorporating hydroxyl terminated HBP in epoxy networks [386-393].

These observations are classically explained by invoking the faster polymerization rate of the AM mechanism in accordance with the rate constants published by Penczek and coworkers (Eq. (11) and Eq. (14)). However, some authors proposed different explanations. They suggested that alcohol may play the role of a co-catalyst. This would result in a more efficient production of initiating species and thus in an acceleration of the overall rate of polymerization, $\mathrm{R}_{\mathrm{p}}$.

As for thermolatent onium salts, Park et al. suggested that alcohols may facilitate the liberation of strong Brønsted acids through the nucleophilic attack of the carbon in $\alpha-$ position of the positively charged metalloid center of the initiator [109]. The mechanism is illustrated in Figure 83 for a thermolatent phosphonium salt.

In the case of photolatent onium salts, several authors demonstrated that alcohols are actually involved in the free-radical-induced chain decomposition of iodonium salts [403-405]. Pappas et al. measured that the quantum yield of the photosensitized decomposition of iodonium salts increased from 0.01 in neat acetonitril to 2.4 in the presence of 2-propanol (acetonitril, 5 mol. $L^{-1}$ ) [404]. Crivello et al. reported a pronounced acceleration of the polymerization rate in the presence of benzyl alcohols [406, 407] and some hydroxymethylated polynuclear aromatic hydrocarbons [408]. Besides the promotion of the AM mechanism, they invoked the involvement of these additives in the free-radical induced chain decomposition of the initiators, namely iodonium salts.

Thus, from aforementioned results, there is no certainty on whether the promotion of the AM mechanism increases or decreases the curing rate of the cationic polymerization of epoxy resins. 
In any case, alcohols appear as valuable additives as they permit to enlarge the variety of initiating systems. When combined with $\mathrm{BF}_{3}$-amine initiators, they promote a decrease of the polymerization rate. On the other hand, in combination with onium salts, they normally involve an increase of the curing rate. Though, it is not always true. For instance, Crivello et al. reported an acceleration of the curing process for the cationic polymerization of aliphatic epoxy resins initiated by an iodonium salt in the presence of ethylene glycol and 1,4butanediol [402]. On the opposite, Wang et al. reported a decrease of the polymerization rate when using a sulfonium salt in the presence of the same alcohols [409]. Similarly, Wang et al. observed opposite variations of the curing rate when using different alcohols with the same initiator [409]. In their study, the use of 1,4-butanediol is accompanied by a decrease of the polymerization rate while pinacol promote the inverse.

These observations demonstrate that the influence of alcohols on cationic polymerization is a rather complex phenomenon that depends on a number of parameters besides the promotion of the AM mechanism. They include the structure of the alcohol [287, 409] as well as the properties of the initiator (e.g. the reduction potential of the onium salt [404-406]).

Eventually, it is important to note that alcohol can play on two different parameters: the curing rate and the conversion at the gel point. If both options may be attractive to program the curing process, it may also be desirable to play with the former while keeping the latter constant. Here lies the limit of the use of alcohol to control the curing kinetics in the cationic mode.

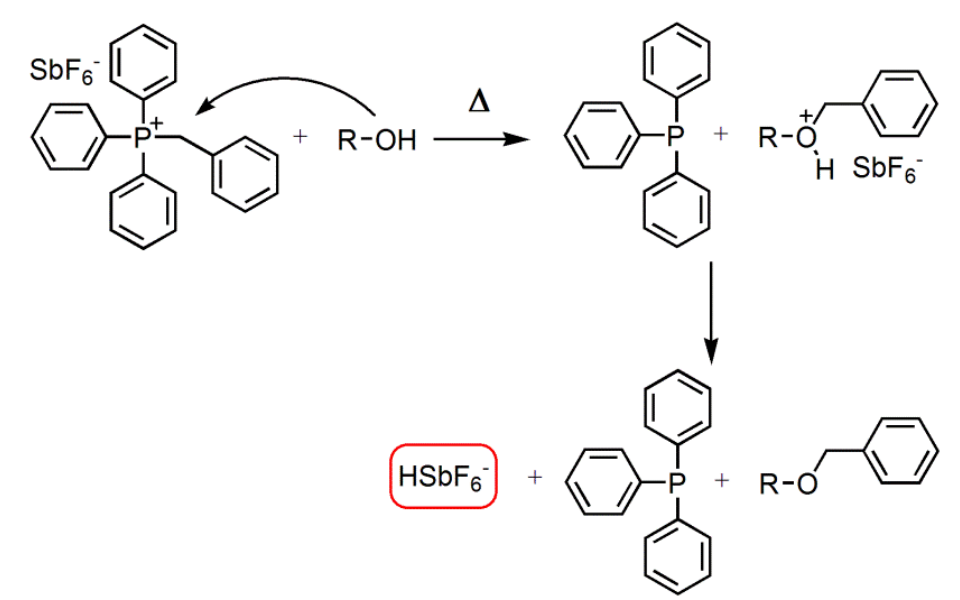

Figure 75: Postulated mechanism for the cocatalytic effect of alcohol with a thermolatent phosphonium salt. 


\subsubsection{Combination of transfers and supramolecular complexation.}

An alternative to the use of diol as retardant are selective chelating ligands. They can complex cationic species and keep under control a reaction which is normally prone to runaway. In section 3.3.3, it was shown that this strategy permits to control the initiation stage when the chelating ligand is the selective 18 -crown-6 ether [283, 366, 372].

Concurrently, several authors have shown that less selective ligands may induce a decrease of the polymerization rate with similar amplitude as alcohols. For instance, Crivello and coworkers suggested the use of multidentate monomers in combination with photoresponsive initiators and showed that stabilization of the photogenerated hydronium ion leads to a better control of the initiation stage [281, 282, 410]. Typical structures of complexes of hydronium and multidentate monomers are illustrated in Figure 84.
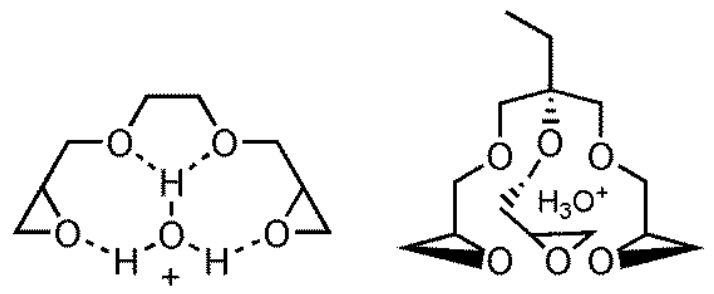

Figure 84: Schematic representation of the tetradentate coordination of a hydronium cation by 1,2-ethanediol diglycidyl ether and of the cage-like complex resulting from the hexadentate coordination of a hydronium cation by the trimethylolpropane triglycidyl ether.

Vidil and Tournilhac used a series of molecules containing hydroxyl and/or ethylene oxide moieties as additives in the cationic polymerization of a standard epoxy resin (DGEBA) initiated by a thermal initiator, the $\mathrm{BF}_{3}$-aromatic amine complex, $\mathrm{BF}_{3}-4 \mathrm{CANH}_{2}$ (see section 3.3.1.2 and 3.3.3) [373]. The additives were judiciously selected to distinguish the contribution of protic groups (alcohols) from the contribution of aprotic groups (ethers). They are depicted in Figure 85. Aprotic polyethers with more than five ethylene oxide patterns in their backbone (Bz-PEO-Bz, Pr-PEO-Pr, Ox-PEO-Ox) induce a significant retardation of the cationic polymerization of DGEBA at $40^{\circ} \mathrm{C}$. Interestingly, the conversion at the gel point is unchanged when compared to the value measured for the additive-free system (about 10$15 \%)$. These results were rationalized by considering the ability of PEO to complex the anilinium cation resulting from the decomposition of the initiator. The structure of the complex is illustrated in Figure 86. Under this protected form, the releasing rate of the Bronsted acid $\mathrm{HBF}_{4}$ is considerably decreased as previously mentioned in Section 3.3.3. 
Thus, unlike alcohols that promote transfers, these additives can be used to control the curing rate without any impact on the conversion at the gel point.

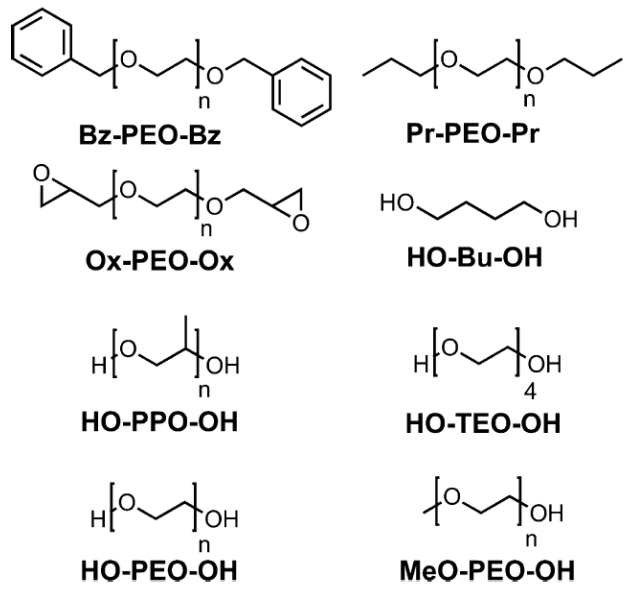

Figure 76: The various polyethers and alcohols additives used by Vidil and Tournilhac $(6<n<8)$ for the curing of DGEBA initiated by $\mathrm{BF}_{3}-4 \mathrm{CANH}_{2}(0.026$ equiv./epoxy) at $40{ }^{\circ} \mathrm{C}[373]$.
Figure 77: Schematic representation of the complexation of the 4-chloroanilinium cation stemming from the initiator $4 \mathrm{CA}-\mathrm{BF}_{3}$ with low molecular weight $\mathrm{PEO}$ endowed with various chain ends, E.

Non-chelating alcohols like butanediol (HO-Bu-OH), polypropylene glycol (HO-PPO-OH) or polyethylene glycol with less than five ethylene oxide units in their backbone (HO-TEO-OH) also induce a retardation of the cationic polymerization of DGEBA initiated by $\mathrm{BF}_{3}-4 \mathrm{CANH}_{2}$. As expected, the conversions at the gel point are shifted to much higher values (30-50\%) proving that, in these cases, the retarding mechanism is the promotion of the AM propagation mode.

Ultimately, Vidil and Tournilhac studied systems combining both hydroxyl groups and complexing polyethers. This situation is illustrated in Figure 87 were the additive-free system is compared with reactive systems containing HO-PEO-OH or a combination of Pr-PEO-Pr and butanediol. In both case, they observed the most pronounced retarding effect of all tested additives with a gel time, $t_{\text {gel }} \approx 70 \mathrm{~min}$ and a high conversion at the gel point, $\mathrm{x}_{\text {gel }} \approx 40 \%$. These observations suggest a superposition of the effects of transfers and of supramolecular complexation, the former being promoted by the hydroxyl groups and the latter resulting from the presence of the polyethylene oxide chains.

Interestingly, almost the same conversion profiles are obtained whether the hydroxyl and chelating polyether functions are carried out by the same molecule (HO-PEO-OH) or by two different molecules (Pr-PEO-Pr + butanediol). 
On this basis, Vidil and Tournilhac proposed a general approach to control the two fundamental parameters of the gelation process for the curing of epoxy resins in the cationic mode, i.e. $t_{\text {gel }}$ and $x_{\text {gel }}$. They demonstrated that it is possible to explore a wide range of $t_{\text {gel }}$ and $\mathrm{X}_{\text {gel }}$ values by adequately adapting the amount of protic (alcohols) and chelating (polyethers) additives. This is well illustrated under the form of a two- dimensional chart, $\mathrm{t}_{\mathrm{gel}} v s$. $\mathrm{x}_{\mathrm{gel}}$, as depicted in Figure 88.

Interestingly, a similar approach has been recently proposed for the polymerization of benzoxadine [411]. Wang et al. demonstrated that the gelation parameters, $\mathrm{t}_{\mathrm{gel}}$ and $\mathrm{x}_{\mathrm{gel}}$, for the thermal curing of Bisphenol A-aniline benzoxazine can be control by using a series of various additives such as phenols, carboxylic acids and imidazoles.

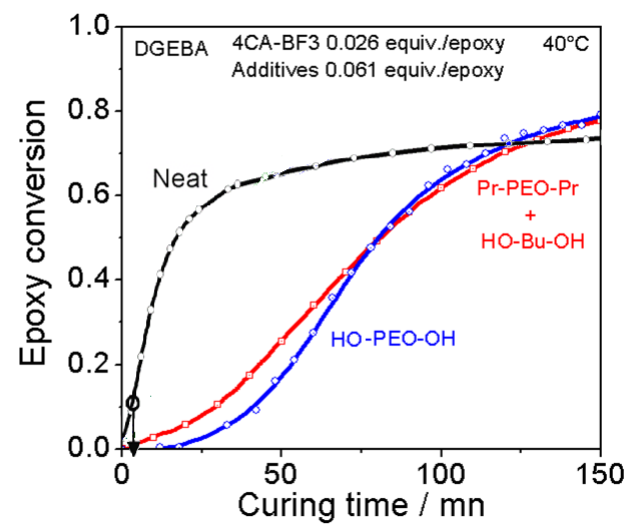

Figure 87: Epoxy conversion profiles obtained for the curing of DGEBA initiated by $4 \mathrm{CA}-\mathrm{BF}_{3} \quad(0.026$ eq./epoxy) in the presence of $\mathrm{HO}-\mathrm{PEO}-\mathrm{OH}, \mathrm{HO}-\mathrm{Bu}-\mathrm{OH}$ and $\mathrm{HO}-\mathrm{PPO}-\mathrm{OH}$ used at 0.061 eq./epoxy at $40^{\circ} \mathrm{C}$ (reproduced after (373) with permission from the American Chemical Society).

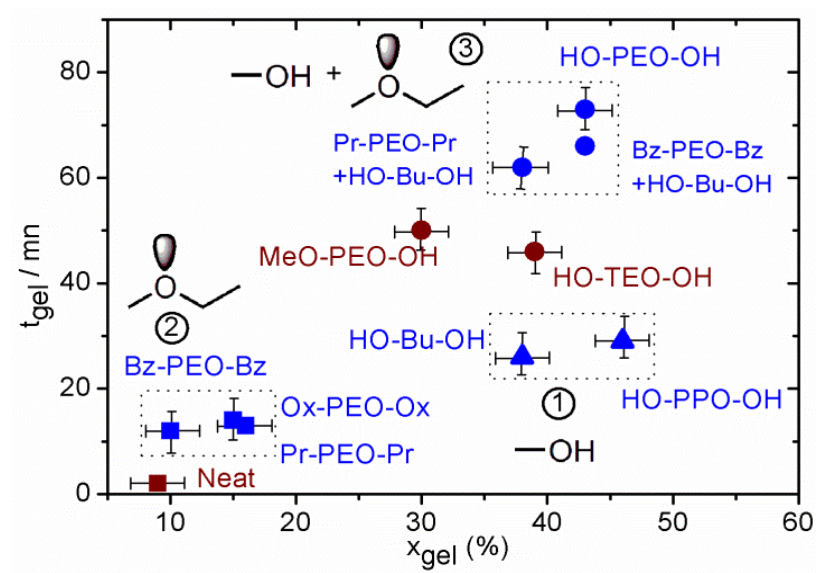

Figure 78: $\left(x_{g e l}, t_{g e l}\right)$ coordinates obtained for additives of various protic and chelating properties (reproduced after (373) with permission from the American Chemical Society).

\subsubsection{Toward clock like curing systems}

This last section exemplifies the possibility to initiate curing after a predictable and controllable induction period in isothermal conditions. It is based on a few unpublished results from Vidil and Tournilhac studies. The strategy is to maintained the reactive centers into a dormant state and to suddenly released them after completion of a reaction that plays the role of an internal timer. In this respect, parallels can be drawn with self-regulated clock-reactions that are characterized by an abrupt increase in the concentration of a chemical after an induction period resulting from the consumption of an inhibiting reagent.

The initiating system studied by Vidil and Tournilhac [373] is based on the combination of 4 chemicals: (i) the Bronsted acid $\mathrm{HBF}_{4}$, (ii) the primary aniline $4 \mathrm{CANH}_{2}$, (iii) a mutidentate 
ligand (linear PEO or crown ether) and (iv) an alcohol. As mentioned in Section 3.3.3, the association of (i) $\mathrm{HBF}_{4}$, (ii) $4 \mathrm{CANH}_{2}$ and (iii) a multidentate ligand results in the formation of a tetrafluoroborate ammonium complexed by the multidentate ligand. As long as the proton is involved in this supramolecular complex, the initiation of the cationic polymerization of the epoxy resin is prevented.

The likelihood to form this complex, and thus to preclude the initiation, is dependent on many parameters including the temperature (see Section 3.3.3), the chelating ability of the ligand (see Section 4.2.4) but also the probability to form the tetrafluoroborate ammonium. Interestingly, when an alcohol is added to this system, the AM mechanism is at work and a proton is transferred after each monomer addition to the growing chains. In these conditions, the probability to observe an acid-base equilibrium involving the Bronsted acid $\mathrm{HBF}_{4}$ and the primary amine $4 \mathrm{CANH}_{2}$ is much larger and so is the probability to form the supramolecular complex with the multidentate ligand.

Thus, curing systems combining the four chemicals proposed by Vidil and Tournilhac (namely $\mathrm{HBF}_{4}, 4 \mathrm{CANH}_{2}$, a mutidentate ligand and an alcohol) are expected to be extensively inhibited. This was already illustrated in section 4.2 .4 with the use of hydroxyl terminated PEO or a combination of alkylated PEO and small non-chelating alcohols (e.g. 1,4butanediol). When using 18-crown-6 instead of a linear polyether, Vidil and Tournilhac observed induction periods as long as 3 days for temperatures as high as $60^{\circ} \mathrm{C}$. The mechanism responsible for this tremendous delay of the cationic polymerization is illustrated in Figure 89. Here, the formation of the very stable $\mathrm{BF}_{4}{ }^{-}-4 \mathrm{CANH}_{3}{ }^{+} \bullet 18$-crown- 6 complex is in competition with the polymerization propagation after each monomer addition.

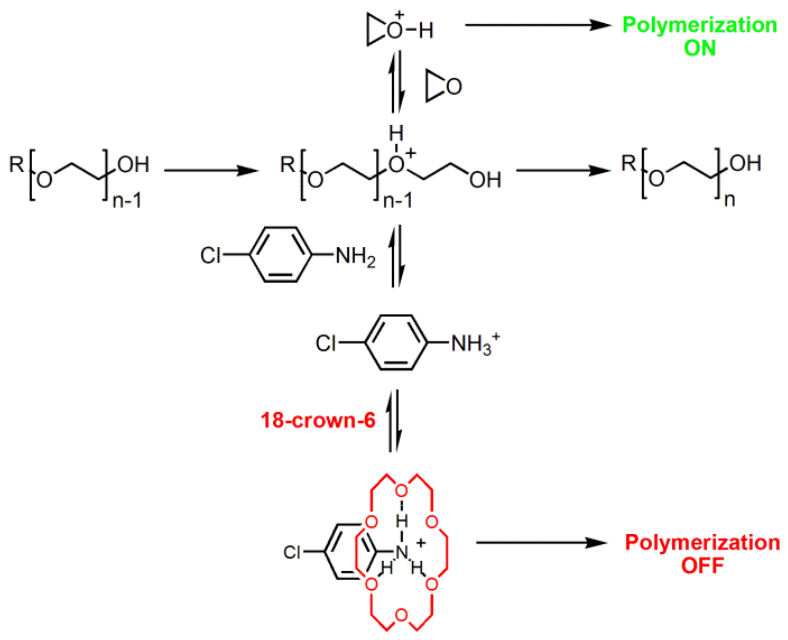

Figure 89: Proposed interpretation for the prolonged retardation period in systems combining 18-crown-6 and $\mathrm{HO}-\mathrm{Bu}-\mathrm{OH}$. Proton exchanged between the linear secondary oxonium ion and the monomer during the activated monomer mechanism can be intercepted by aniline. In the presence of 18-crown-6, the resulting anilinium cation is complexed and protons are trapped in the supramolecular complex. Polymerization is turned off. 
Despite this drastic inhibiting mechanism, Vidil and Tournilhac observed that polymerization restarts after a period dependent on the proportion of the 4 chemicals involved in the initiating system. The conversion profiles of the curing systems are strongly non-linear and the polymerization rate suddenly jump from nearly 0 to values classically measured for the additive-free system. Similar conversion profiles are observed for clock-reactions. In this case, the abrupt jump of the conversion rate is usually obtained after exhaustion of an inhibiting reagent that is consumed through a side reaction. This parallel suggests that a side reaction may be responsible for the exhaustion of one of the 4 chemicals involved in the initiating system proposed by Vidil and Tournilhac.

In their study of the cationic polymerization of epoxides initiated by $\mathrm{BF}_{3}$-amine complexes, Chabanne and coworkers demonstrated that the amine stemming from the initiator readily reacts with the activated monomer [105]. This addition process is not reversible; it produces a secondary amine whose conjugated ammonium is bulkier and less symmetrical than its primary analogue. Under this form, it can no longer form a complex with the crown ether. The situation is summarized in Figure 90. After exhaustion of the primary amine, one of the compulsory chemicals for the sequestration of the proton is missing and $\mathrm{HBF}_{4}$ is free to participate in polymerization initiation and transfers.

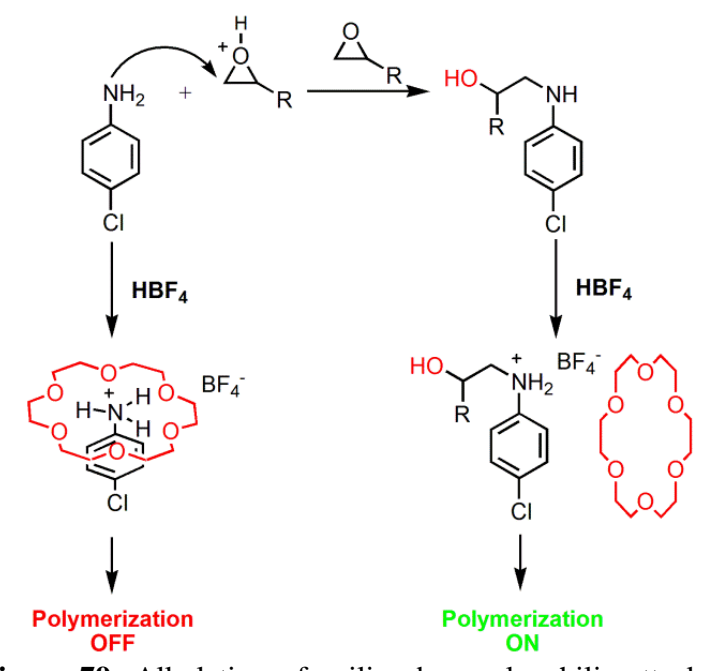

Figure 79: Alkylation of aniline by nucleophilic attack of the activated monomer. The resulting secondary aniline cannot bind to 18-crown-6 after protonation. Protons are no longer trapped in supramolecular complexes and the polymerization rate increases.

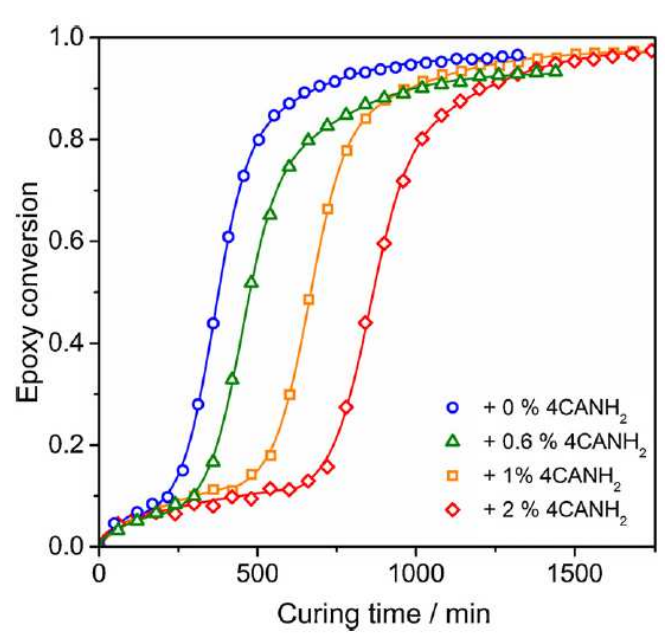

Figure 91: Epoxy conversion profiles obtained for the curing of DGEBA initiated by $\mathrm{BF} 3-4 \mathrm{CANH}_{2} \quad(0.026$ equiv./epoxy) in the presence of a combination of 18-crown6 and $\mathrm{HO}-\mathrm{Bu}-\mathrm{OH}$ both used at 0.061 equiv./epoxy at $40{ }^{\circ} \mathrm{C}$ and with the addition of small portions of $4 \mathrm{CANH}_{2}$.

Vidil and Tournilhac demonstrated that this system may be used to control the duration of the induction period by varying the initial concentration of primary amine in the curing system. Figure 91 illustrates the evolution of the conversion profiles for the curing of DGEBA at 
$60^{\circ} \mathrm{C}$, initiated by $\mathrm{BF}_{3}-4 \mathrm{CANH}_{2}$ (0.026 equiv./epoxy) in the presence of 1,4-butanediol (0.061 equiv./epoxy) and 18-crown-6 (0.061 equiv./epoxy) and with increasing amounts of the aniline $4 \mathrm{CANH}_{2}$. It is based on results published in [45]. Results show that the induction period can be controlled to a great extent with duration varying from 200 to more than 700 min $(=11 \mathrm{~h})$ by adding small portions of $4 \mathrm{CANH}_{2}$ comprised between 0 and $2 \%$ (where $1 \%$ means 0.01 eq./epoxy). Thus, the gradual consumption of aniline permits the design of self regulated systems that polymerize after a predictable and controllable period of time in isothermal condition.

This clock-like behavior may be useful to control the pot life of curing formulations in injection, molding or coating applications. Advantageously, it could be used to program the cure of epoxy resins placed in remote areas where it is not possible to trigger the reaction with an exogenous stimulus like light or temperature.

\section{Conclusions and perspectives}

The versatility of epoxy resins and their remarkable mechanical performances are commensurate with the richness of the chemistry described in this review. In polymer chemistry, apart from olefins, very few chemical functions have such a vast range of options at disposal to control the initiation and the growth of macromolecules. In the case of epoxy resins, an arsenal of latent, inhibited or delayed curing systems has been developed to address the technical challenges related to processing and shaping.

First of all, the chemistry of epoxy thermosets is rich of a number of latent polymerization systems. Today, chemists have at their disposal not only the first generation of latent systems based on biphasic reactive mixtures (e.g. high melting point hardeners) but also new approaches based on the controlled release of reactive molecules following the breaking of covalent bonds. Hydrolyzable, photo- or thermo-cleavable molecules are used to initiate curing reactions upon exposure to a stimulus. Concurrently, triggerable systems based on the stabilization of reactive molecules within supramolecular scaffolds have been investigated. Advantageously, this strategy — based on non-covalent bonds - offers thermo-activated systems working in much lower temperature ranges than conventional thermolatent initiators that rely on cleavable covalent bonds.

Meanwhile, the need to control the hardening of the resin once the reaction is initiated led to the development of chemical strategies dedicated to the control of the propagation stage. 
Indeed, chain transfer agents are conventionally added to the reactive mixtures in order to prevent the runaway conditions of cationic and anionic ROP. These are usually alcohols that strongly interfere in the propagation mechanism by promoting transfers. In Brønsted acid initiated systems, alcohols delay the onset of the gel point by slowing down the rate of polymerization and reducing the size of the growing chains. Thus, aside from the control of the curing kinetics, alcohols enable the control of the growing network's structure. Recently, in cationic polymerization, it was demonstrated that transfers can be intercepted by the stabilization of protons in supramolecular structures. This new approach allows for the development of clock-like curing systems, i.e. whose curing is inhibited and restart after a known induction period. Moreover, it provides epoxide's polymerization with a counterpart to the strategies used in controlled polymerization of olefins where free radicals are stabilized in dormant states. In this sense, this approach is likely to be the premise of the development of new controlled polymerization methods for epoxides and other cyclic ethers.

Overall, the chemistry of epoxy thermosets is moving on with modern chemistry of polymers. It is part of a broader movement dedicated to stimuli-responsive polymeric systems. As an illustration, the recent discovery of vitrimers supports well the idea that a simple but robust chemistry may feed the forefront of technological innovations in material science. In this review, it appears that the oldest and most challenging technical issues associated with the use of thermosets - i.e. the control of both the initiation and the crosslinking kinetics - remains a field of active researches to meet the needs of emerging technologies (e.g. self-repairing materials, 3D printing...) and the new environmental requirements (energy saving curing systems). It led to the development of a rich and diverse chemistry that can be a source of inspiration in other areas of macromolecular engineering.

\section{Acknowledgement}

Thomas Vidil thanks Schlumberger for financial support

\section{References}

1 L. H. Baekeland The synthesis, constitution, and uses of bakelite Ind. Eng. Chem. 1909 ;1: 149-161.

2 J.-P. Pascault, H. Sautereau, J. Verdu and R. J. J. Williams Thermosetting Polymers New York: Marcel Dekker 2002496 pp.

3 K. Van de Velde and P. Kiekens Thermoplastic polymers: overview of several properties and their consequences in flax fibre reinforced composites Polym. Test. 2001 ;20: 885-893.

$4 \quad$ D. W. van Krevelen and K. te Nijenhuis Properties of polymers Amsterdam: Elsevier 20091032 pp. 
C. Y. M. Tung and P. J. Dynes Relationship between viscoelastic properties and gelation in thermosetting systems J. Appl. Polym. Sci. 1982 ;27: 569-574.

H. H. Winter Can the gel point of a cross-linking polymer be detected by the G' - G" crossover? Polym. Eng. Sci. 1987 ;27: 1698-1702.

D. H. Kim and S. C. Kim Engineering analysis of reaction injection-molding process of epoxy-resin Polym. Compos. 1987 ;8: 208-217.

M. Naffakh, M. Dumon and J.-F. Gerard Modeling the chemorheological behavior of epoxy/liquid aromatic diamine for resin transfer molding applications J. Appl. Polym. Sci. 2006 ;102: 4228-4237.

Y. Ding, Y. Jia, J. Yang, H. He, S. Sun and T. Shi Correlation analysis of epoxy/amine resin cure, structure and chemorheological behavior in RTM processes Polym. Compos. 2011 ;32: 648-656.

J. S. Osinski and L. T. Manzione Characterization and moldability analysis of epoxy reaction injection-molding resins ACS Symp. Ser. 1983 ;221: 263-282.

D. Feldman Some considerations on thermosetting polymers as matrices for composites Prog. Polym. Sci. 1990 ;15: 603-628.

L. M. Bonnaillie and R. P. Wool Thermosetting foam with a high bio-based content from acrylated epoxidized soybean oil and carbon dioxide J. Appl. Polym. Sci. 2007 ;105: 1042-1052.

S. R. White, J. S. Moore, N. R. Sottos, B. P. Krull, W. A. S. Cruz and R. C. R. Gergely Restoration of Large Damage Volumes in Polymers Science 2014 ;344: 620-623.

A. L. Rutz, K. E. Hyland, A. E. Jakus, W. R. Burghardt and R. N. Shah A multimaterial bioink method for 3D printing tunable, cell-compatible hydrogels Adv. Mater. 2015 ;27: 1607-1614.

C. A. May, editor. Epoxy resins: Chemistry and technology New York: Marcel Dekker 19881242 pp.

J. P. Pascault and R. J. J. Williams Epoxy polymers: New materials and innovations Weinheim: Wiley 2010384 pp. 
E. W. Flick Epoxy resins, curing agents, compounds, and modifiers: an industrial guide New Jersey: Noyes publication 1993519 pp.

27 L. Chiao Mechanistic reaction-kinetics of 4,4'-diaminodiphenyl sulfone cured tetraglycidyl- 4,4'diaminodiphenylmethane epoxy-resins Macromolecules 1990 ;23: 1286-1290.

T. H. Hsieh and A. C. Su Cure kinetics of an epoxy-novolac molding compound J. Appl. Polym. Sci. 1990 ;41: 1271-1280.

S. P. Rwei, A. Y. Liu, G. S. Liou, K. C. Cheng and W. Guo Curing and pyrolysis of cresol novolac epoxy resins containing 2-(6-oxido-6H-dibenz(c,e) (1,2)oxaphosphorin-6-yl)-1,4-naphthalenediol Polym. Eng. Sci. 2004 ;44: 376 387.

A. R. Meath Chemistry, properties and applications of epoxy Novolac, flexible epoxy and flame retardant epoxy resins in: P. F. Bruins, editor. Epoxy resin technology New York: Interscience 1968 pp. 31-44. J. J. Stevens Chemistry, properties and applications of cycloaliphatic epoxy resins in: P. F. Bruins, editor. Epoxy Resin Technology New York: Interscience 1968 pp. 11-30.

Y. Morita Cationic polymerization of hydrogenated bisphenol-A glycidyl ether with cycloaliphatic epoxy resin and its thermal discoloration J. Appl. Polym. Sci. 2005 ;97: 1395-1400.

A. R. Spurr A low viscosity epoxy resin embedding medium for electron microscopy J. Ultrastruct. Res. $1969 ; 26$ : $31-43$.

W. Liu, Z. Wang, L. Xiong and L. Zhao Phosphorus-containing liquid cycloaliphatic epoxy resins for reworkable environment-friendly electronic packaging materials Polymer 2010 ;51: 4776-4783.

S. Yang, J. S. Chen, H. Korner, T. Breiner, C. K. Ober and M. D. Poliks Reworkable epoxies: Thermosets with thermally cleavable groups for controlled network breakdown Chem. Mater. 1998 ;10: 1475-1482.

E. M. Petrie Epoxy adhesive formulations New York: McGraw-Hill 2006535 pp. UK, Glasgow: Blackie academic \& professional 1993 pp. 117-143.

S. Montserrat, G. Andreu, P. Cortes, Y. Calventus, P. Colomer, J. M. Hutchinson and J. Malek Addition of a reactive diluent to a catalyzed epoxy-anhydride system .1. Influence on the cure kinetics J. Appl. Polym. Sci. 1996 ;61: 1663-1674. N. V. Tsarevsky, S. A. Bencherif and K. Matyjaszewski Graft copolymers by a combination of ATRP and two different consecutive click reactions Macromolecules 2007 ;40: 4439-4445. W. R. Ashcroft Curing agents for epoxy resins in: B. Ellis, editor. Chemistry and technology of epoxy resins UK, Glasgow: Blackie academic \& professional 1993 pp. 37-71. K. Dusek, M. Ilavsky and S. Lunak Curing of epoxy-resins .1. Statistics of curing of diepoxides with diamines J. Polym. Sci. Polym. Polym. Symp. 1975 ;53: 29-44. S. Lunak and K. Dusek Curing of epoxy-resins .2. Curing of bisphenol a diglycidyl ether with diamines J. Polym. Sci. Polym. Polym. Symp. 1975 ;53: 45-55. K. Dusek and M. llavsky Formation, structure, and elasticity of loosely crosslinked epoxy-amine networks .1. Statistics of formation J. Polym. Sci., Part B: Polym. Phys. 1983 ;21: 1323-1339. Y. G. Lin, H. Sautereau and J. P. Pascault Processing property relationship for dicyandiamide-cured epoxy networks J. Appl. Polym. Sci. 1987 ;33: 685-691. 

epoxy-amine system by means of dynamic rheology .2. High-Tg epoxy-amine system Macromolecules 1996 ;29: 6917-6927.

D. A. Unruh, S. J. Pastine, J. C. Moreton and J. M. J. Frechet Thermally activated, single component epoxy systems Macromolecules $2011 ; 44:$ 6318-6325. K. Suzuki, N. Matsu-ura, H. Horii, Y. Sugita, F. Sanda and T. Endo One-pot curing system of epoxy resin imines initiated with water J. Appl. Polym. Sci. 2003 ;88: 878-882.

B. A. Rozenberg Kinetics, thermodynamics and mechanism of reactions of epoxy oligomers with amines $A d v$. Polym. Sci. 1986 ;75: 113-165.

D. Peters Theory of the ether linkage in: S. Patai, editor. The chemistry of the ether linkage UK, London: Interscience 1967 pp. 1-19.

R. J. Gritter Reaction of cyclic ethers in: S. Patai, editor. The chemistry of the ether linkage UK, London: Interscience 1967 pp. 373-443.

M. S. Heise and G. C. Martin Curing mechanism and thermal-properties of epoxy imidazole systems Macromolecules 1989 ;22: 99-104.

V. Jisova Curing mechanism of epoxides by imidazoles J. Appl. Polym. Sci. 1987 ;34: 2547-2558.

L. Matejka, S. Pokorny and K. Dusek Acid curing of epoxy-resins - a comparison between the polymerization of diepoxide-diacid and monoepoxide-cyclic anhydride systems Makromol. Chem. Macromol. Chem. Phys. 1985 ;186: 2025-2036.

K. Dusek, S. Lunak and L. Matejka Gelation in the curing of epoxy-resins with anhydrides Polym. Bull. ;7: 145-152.

J. Rocks, L. Rintoul, F. Vohwinkel and G. George The kinetics and mechanism of cure of an amino-glycidyl epoxy resin by a co-anhydride as studied by FT-Raman spectroscopy Polymer 2004 ;45: 6799-6811.

X. Fernandez-Francos, X. Ramis and A. Serra From curing kinetics to network structure: A novel approach to the modeling of the network buildup of epoxy-anhydride thermosets J. Polym. Sci., Part A: Polym. Chem. 2014 ;52: 61 75.

S. Ohsawa, K. Morino, A. Sudo and T. Endo Synthesis of a reactive polyester bearing alpha,beta-unsaturated ketone groups by anionic alternating copolymerization of epoxide and bicyclic bis(gamma-butyrolactone) bearing isopropenyl group Macromolecules 2011 ;44: 1814-1820.

K. Chung, T. Takata and T. Endo Anionic cross-linking of polymers having an epoxy group in the side chain with bicyclic and spirocyclic bis(gamma-lactone)s Macromolecules 1997 ;30: 2532-2538.

K. Uenishi, A. Sudo and T. Endo Synthesis of polyester having sequentially ordered two orthogonal reactive groups by anionic alternating copolymerization of epoxide and bislactone J. Polym. Sci., Part A: Polym. Chem. 2009 ;47: 6750-6757.

X. Fernandez-Francos, J. M. Salla, A. Mantecon, A. Serra and X. Ramis Crosslinking of mixtures of DGEBA with 1,6-dioxaspiro 4,4 nonan-2,7-dione initiated by tertiary amines. I. Study of the reaction and kinetic analysis J. Appl. Polym. Sci. 2008 ;109: 2304-2315.

X. Fernandez-Francos, J. M. Salla, A. Mantecon, A. Serra and X. Ramis Crosslinking of mixtures of DGEBA with 1,6-dioxaspiro 4.4 nonan-2,7-dione initiated by tertiary amines, Part II: Thermo-mechanical properties and reworkability Polym. Degrad. Stab. 2008 ;93: 760-769. 
X. Fernandez-Francos, W. D. Cook, A. Serra, X. Ramis, G. G. Liang and J. M. Salla Crosslinking of mixtures of DGEBA with 1,6-dioxaspiro 4,4 nonan-2,7-dione initiated by tertiary amines. Part IV. Effect of hydroxyl groups on initiation and curing kinetics Polymer $2010 ; 51: 26-34$.

N. Bouillon, J. P. Pascault and L. Tighzert Epoxy prepolymers cured with boron trifluoride-amine complexes 1. Influence of the amine on the curing window Makromol. Chem. Macromol. Chem. Phys. 1990 ;191: 1403-1416. Y. Yagci and I. Reetz Externally stimulated initiator systems for cationic polymerization Prog. Polym. Sci. $1998 ; 23$ : 1485-1538.

J. V. Crivello and J. H. W. Lam Diaryliodonium salts - new class of photo-initiators for cationic polymerization Macromolecules 1977 ;10: 1307-1315.

J. V. Crivello and J. H. W. Lam Photoinitiated cationic polymerization with triarylsulfonium salts J. Polym. Sci., Part A: Polym. Chem. 1979 ;17: 977-999.

K. Takuma, T. Takata and T. Endo Cationic polymerization of epoxide with benzyl phosphonium salts as the latent thermal initiator Macromolecules 1993 ;26: 862-863.

T. Toneri, K. Watanabe, F. Sanda and T. Endo Synthesis and the initiator activity of fluorenyltriphenylphosphonium salts in the cationic polymerization of epoxide. Novel thermally latent initiators Macromolecules 1999 ;32: 12931296.

L. Matejka, P. Chabanne, L. Tighzert and J. P. Pascault Cationic polymerization of diglycidyl ether of bisphenol-A J. Polym. Sci., Part A: Polym. Chem. 1994 ;32: 1447-1458.

C. Mas, X. Ramis, J. M. Salla, A. Mantecon and A. Serra Copolymerization of diglycidyl ether of bisphenol a with gamma-butyrolactone catalyzed by ytterbium triflate: Shrinkage during curing J. Polym. Sci., Part A: Polym. Chem. $2003 ; 41: 2794-2808$.

X. Fernandez, J. M. Salla, A. Serra, A. Mantecon and X. Ramis Cationic copolymerization with a spirobislactone with of cycloaliphatic epoxy resin lanthanum triflate as initiator: I. Characterization and shrinkage J. Polym. Sci., Part A: Polym. Chem. 2005 ;43: 3421-3432.

P. J. Flory Molecular size distribution in three dimensional polymers. I. Gelation J. Am. Chem. Soc. 1941 ;63: 30833090.

T. Vidil Control of the curing process and the mechanical properties of epoxy resins via supramolecular chemistry [Thesis] Paris: Université Pierre et Marie Curie 2015346 pp. K. Dusek Are cured thermoset resins inhomogeneous? Angew. Makromol. Chem. 1996 ;240: 1-15. Prog. Org. Coat. 2000 ;25: 1215-1260.

C. Katahira, N. Morishita, J.-I. Ikeda, P. B. Lim, M. Inoue, Y. Iwasaki, H. Aota and A. Matsumoto Mechanistic discussion of cationic crosslinking copolymerizations of 1,2-epoxycyclohexane with diepoxide crosslinkers accompanied by intramolecular and intermolecular chain transfer reactions J. Polym. Sci., Part A: Polym. Chem. $2010 ; 48: 4445-4455$.

H. Gao and K. Matyjaszewski Synthesis of functional polymers with controlled architecture by CRP of monomers in the presence of cross-linkers: From stars to gels Prog. Polym. Sci. 2009 ;34: 317-350. combining isoconversional analysis with temperature modulated DSC and dynamic rheometry Macromol. Chem. Phys. 2003 ;204: 1815-1821. 
D. H. Kim and S. C. Kim Vitrification effect during the reaction injection-molding (RIM) process of epoxy-resin Polym. Eng. Sci. 1989 ;29: 456-462.

D. Montarnal, M. Capelot, F. Tournilhac and L. Leibler Silica-like malleable materials from permanent organic networks Science 2011 ;334: 965-968.

M. Capelot, M. M. Unterlass, F. Tournilhac and L. Leibler Catalytic control of the vitrimer glass transition ACS Macro Lett. 2012 ;1: 789-792.

M. Capelot, D. Montarnal, F. Tournilhac and L. Leibler Metal-catalyzed transesterification for healing and assembling of thermosets J. Am. Chem. Soc. 2012 ;134: 7664-7667.

A. Maazouz, J. Dupuy and G. Seytre Polyurethane and unsaturated polyester hybrid networks: Chemorheological and dielectric study for the resin transfer molding process (RTM) Polym. Eng. Sci. 2000 ;40: 690-701. H. Ishida and D. A. Zimmerman The development of an epoxy-resin system for the injection-molding of long-fiber epoxy composites Polym. Compos. 1994 ;15: 93-100. A. Mateus, P. J. Bartolo, G. Mitchell, M. Silva, A. S. Pouzada and A. J. Pontes The effect of thermal conductivity of RIM moulds in kinetics cure in: J. d. Paulo and M. A. Jorge, editor. Virtual and Rapid Manufacturing: Advanced Research in Virtual and Rapid Prototyping Leiden:Taylor \& Francis 2007 389-394. W. Zhao, A. Pizzi, V. Fierro, G. Du and A. Celzard Effect of composition and processing parameters on the characteristics of tannin-based rigid foams. Part I: Cell structure Mater. Chem. Phys. 2010 ;122: 175-182. W. Zhao, V. Fierro, A. Pizzi, G. Du and A. Celzard Effect of composition and processing parameters on the characteristics of tannin-based rigid foams. Part II: Physical properties Mater. Chem. Phys. 2010 ;123: 210-217. V. Rebizant, A. S. Venet, F. Tournilhac, E. Girard-Reydet, C. Navarro, J. P. Pascault and L. Leibler Chemistry and mechanical properties of epoxy-based thermosets reinforced by reactive and nonreactive SBMX block copolymers Macromolecules 2004 ;37: 8017-8027.

H. Sun, Y. Liu, Y. Wang and H. Tan Curing behavior of epoxy resins in two-stage curing process by non-isothermal differential scanning calorimetry kinetics method J. Appl. Polym. Sci. 2014 ;131: 40711/1-8.

A. T. Detwiler and A. J. Lesser Characterization of double network epoxies with tunable compositions J. Mater. Sci. 2012 ; 47: 3493-3503.

J. B. Enns and J. K. Gillham Time temperature transformation (TTT) cure diagram - modeling the cure behavior of thermosets J. Appl. Polym. Sci. 1983 ;28: 2567-2591. L. C. Chan, H. N. Nae and J. K. Gillham Time-temperature transformation (TTT) diagrams of high Tg epoxy systems - competition between cure and thermal-degradation J. Appl. Polym. Sci. 1984 ;29: 3307-3327. D. Verchere, H. Sautereau, J. P. Pascault, C. C. Riccardi, S. M. Moschiar and R. J. J. Williams Buildup of epoxycycloaliphatic amine networks - kinetics, vitrification, and gelation Macromolecules 1990 ;23: 725-731. D. Santiago, X. Fernandez-Francos, X. Ramis, J. M. Salla and M. Sangermano Comparative curing kinetics and thermal-mechanical properties of DGEBA thermosets cured with a hyperbranched poly(ethyleneimine) and an aliphatic triamine Thermochim. Acta 2011 ;526: 9-21.

S. Naumann and M. R. Buchmeiser Latent and delayed action polymerization systems Macromol. Rapid Commun. 2014 ;35: 682-701.

T. Endo and F. Sanda Design of latent catalysts and their application to polymer synthesis Macromol. Symp. 1996 ;107: 237-242. 
G. Odian Principles of Polymerization Hoboken, NJ: Wiley 2004812 pp..

S. Finson, J. M. Jury and J. V. Crivello Azodioxides as inhibitors and retarders in photoinitiated cationic polymerization Macromol. Chem. Phys. 2013 ;214: 1806-1816.

F. A. Bovey and I. M. Kolthoff Inhibition and retardation of vinyl polymerization Chem. Rev. 1948 ;42: 491-525.

101 L. Li and L. J. Lee Effects of inhibitors and retarders on low temperature free radical crosslinking polymerization between styrene and vinyl ester resin Polym. Eng. Sci. 2001 ;41: 53-65.

M. Tejkl, J. Valis, M. Kaplanova, B. Jasurek and T. Syrovy Inhibition of premature polymerization of cationically polymerizable low viscosity systems Prog. Org. Coat. $2012 ; 74:$ 215-220.

S. K. Rath, A. Diby, Z. Seghier, F. Y. C. Boey and M. J. M. Abadie The effect of amines on the UV-curing of epoxy resins Iran. Polym. J. 2006 ;15: 855-862.

04 R. Harikrishna Inhibition effect of $\mathrm{N}, \mathrm{N}$-diglycidyl-4-glycidyloxy aniline on photosensitized cationic polymerization of formulations involving resorcinol diglycidyl ether and poly(propylene glycol)diglycidyl ether J. Photochem.

Photobiol. A Chem. 2015 ;303: 17-27.

105 P. Chabanne, L. Tighzert and J. P. Pascault Monoepoxy polymerization initiated by BF3-amine complexes in bulk .2. Influence of water and by-products on polymer formation J. Appl. Polym. Sci. 1994 ;53: 769-785.

Z. Chen, Y. Zhang, B. J. Chisholm and D. C. Webster A humidity blocker approach to overcoming the humidity interference with cationic photopolymerization J. Polym. Sci., Part A: Polym. Chem. 2008 ;46: 4344-4351.

A. Hartwig, B. Schneider and A. Luhring Influence of moisture on the photochemically induced polymerisation of epoxy groups in different chemical environment Polymer 2002 ;43: 4243-4250.

108 A. Hartwig Influence of moisture present during polymerisation on the properties of a photocured epoxy resin Int. J. Adhes. Adhes. 2002 ;22: 409-414.

109 S. J. Park, G. Y. Heo and D. H. Suh Thermal properties and fracture toughness of epoxy resins cured by phosphonium and pyrazinium salts as latent cationic initiators J. Polym. Sci., Part A: Polym. Chem. 2003 ;41: 2393-2403 .

110 J. V. Crivello and L. Bulut Dual photo- and thermally initiated cationic polymerization of epoxy monomers J. Polym. Sci., Part A: Polym. Chem. 2006 ;44: 6750-6764.

111 D. Foix, X. Ramis, A. Serra and M. Sangermano UV generation of a multifunctional hyperbranched thermal crosslinker to cure epoxy resins Polymer $2011 ; 52:$ 3269-3276.

112 C. Block, B. Van Mele, P. Van Puyvelde and G. Van Assche Time-temperature-transformation (TTT) and temperature-conversion-transformation (TxT) cure diagrams by RheoDSC: Combined rheometry and calorimetry on an epoxy-amine thermoset React. Funct. Polym. 2013 ;73: 332-339.

113 M. S. Genidy, M. S. Madhukar and J. D. Russell An investigation of cure induced stresses in law cure temperature thermoset polymer composites J. Reinf. Plast. Compos. 1999 ;18: 1304-1321.

114 M. Morell, A. Lederer, X. Ramis, B. Voit and A. Serra Multiarm star poly(glycidol)-block-poly(epsilon-caprolactone) of different arm lengths and their use as modifiers of diglycidylether of bisphenol a thermosets J. Polym. Sci., Part A: Polym. Chem. $2011 ; 49:$ 2395-2406.

115 D. R. Miller, E. M. Valles and C. W. Macosko Calculation of molecular-parameters for stepwise polyfunctional polymerization Polym. Eng. Sci. 1979 ;19: 272-283.

116 M. Blanco, M. Lopez, P. Alvarez De Arcaya, J. Angel Ramos, G. Kortaberria, C. Cristina Riccardi and I. Mondragon Thermoplastic-modified epoxy resins cured with different functionalities amine mixtures: Morphology, thermal behavior, and mechanical properties J. Appl. Polym. Sci. 2009 ;114: 1753-1760. 
A. Tcharkhtchi, A. S. Lucas, J. P. Trotignon and J. Verdu Viscoelastic properties of epoxy networks in the glass transition region Polymer 1998 ;39: 1233-1235.

118 A. Matsumoto Free-radical crosslinking polymerization and copolymerization of multivinyl compounds Adv. Polym. Sci. 1995 ;123: 41-80.

119 L. Matejka, K. Dusek, P. Chabanne and J. P. Pascault Cationic polymerization of diglycidyl ether of bisphenol A .2. Theory J. Polym. Sci., Part A: Polym. Chem. 1997 ;35: 651-663.

120 L. Matejka, K. Dusek, P. Chabanne and J. P. Pascault Cationic polymerization of diglycidyl ether of bisphenol A .3. Comparison of the theory with experiment J. Polym. Sci., Part A: Polym. Chem. 1997 ;35: 665-672.

$121 \mathrm{X}$. Fernandez-Francos Theoretical modeling of the effect of proton donors and regeneration reactions in the network build-up of epoxy thermosets using tertiary amines as initiators Eur. Polym. J. 2014 ;55: 35-47.

122 A. N. Mauri, N. Galego, C. C. Riccardi and R. J. J. Williams Kinetic model for gelation in the diepoxide-cyclic anhydride copolymerization initiated by tertiary amines Macromolecules 1997 ;30: 1616-1620.

123 H. H. Winter and M. Mours Rheology of polymers near liquid-solid transitions Adv. Polym. Sci. 1997 ;134: 165-234.

124 K. Dusek, H. Galina and J. Mikes Features of network formation in the chain crosslinking (co)polymerization Polym. Bull. $\quad 1980 ; 3: 19-25$.

125 S. Penczek, P. Kubisa and R. Szymanski Activated monomer propagation in cationic polymerizations Makromol. Chem. Macromol. Symp. 1986 ;3: 203-220.

126 A. Sabra, T. M. Lam, J. P. Pascault, M. F. Grenier-Loustalot and P. Grenier Characterization and behavior of epoxy-based diaminodiphenylsulfone networks Polymer 1987 ;28: 1030-1036.

127 M. J. Marks and R. V. Snelgrove Effect of conversion on the structure-property relationships of amine-cured epoxy thermosets ACS Appl. Mater. Interfaces 2009 ;1: 921-926.

128 M. D. Gilbert, N. S. Schneider and W. J. Macknight Mechanism of the dicyandiamide epoxide reaction Macromolecules 1991 ;24: 360-369.

129 M. Fedtke, F. Domaratius, K. Walter and A. Pfitzmann Curing of epoxy-resins with dicyandiamide - model investigations with substituted dicyandiamides Polym. Bull. 1993 ;31: 429-435.

130 M. Fedtke, F. Domaratius and A. Pfitzmann Curing of epoxy-resins with dicyandiamide - reaction behavior of glycidyl ethers Polym. Bull. 1990 ;23: 381-388.

131 T. Guthner and B. Hammer Curing of epoxy-resins with dicyandiamide and urones J. Appl. Polym. Sci. $1993 ; 50$ : 1453-1459.

132 N. Amdouni, H. Sautereau, J. F. Gerard and J. P. Pascault Epoxy networks based on dicyandiamide - effect of the cure cycle on viscoelastic and mechanical-properties Polymer $1990 ; 31$ : 1245-1253.

133 X. M. Chen and B. Ellis Coatings and other applications of epoxy resins in: B. Ellis, editor. Chemistry and technology of epoxy resins UK, Glasgow: Blackie Academic \& professional 1993 pp. 303-326.

134 S. J. Garcia, A. Serra, X. Ramis and J. Suay Influence of the addition of erbium and ytterbium triflates in the curing kinetics of a DGEBA/o-tolybiguanide powder mixture J. Therm. Anal. Calorim. 2007 ;89: 223-231.

135 A. M. Tomuta, X. Ramis, F. Ferrando and A. Serra The use of dihydrazides as latent curing agents in diglycidyl ether of bisphenol A coatings Prog. Org. Coat. 2012 ;74: 59-66.

136 A. M. Tomuta, X. Ramis, S. de la Flor and A. Serra Influence of end groups in hyperbranched polyesters used as modifiers in the characteristics of epoxy thermosets cured by adipic dihydrazide Express. Polym. Lett. $2013 ; 7$ : 595-606. 

polymers made from oligoisoprene dihydrazides and epoxide oligomer Polym. Sci. U.S.S.R 1983 ;25: 1063-1074. L. Matejka and K. Dusek Specific features of the kinetics of addition esterification of epoxide with the carboxyl group Polym. Bull. 1986 ;15: 215-221.

139 L. Schechter, J. Wynstra and R. Kurkjy Chemistry of styrene oxide. Comparison with phenyl glycidyl ether in model compound reactions Ind. Eng. Chem. 1957 ; 49: 1107-1109.

140 W. J. Blank, Z. A. He and M. Picci Catalysis of the epoxy-carboxyl reaction J. Coat. Technol. 2002 ;74: 33-41.

141 G. Merfeld, C. Molaison, R. Koeniger, A. E. Acar, S. Mordhorst, J. Suriano, P. Irwin, R. S. Warner, K. Gray, M. Smith, K. Kovaleski, G. Garrett, S. Finley, D. Meredith, M. Spicer and T. Naguy Acid/epoxy reaction catalyst screening for low temperature $\left(120^{\circ} \mathrm{C}\right)$ powder coatings Prog. Org. Coat. $2005 ; 52:$ 98-109.

142 M. Pire, S. Norvez, I. lliopoulos, B. Le Rossignol and L. Leibler Imidazole-promoted acceleration of crosslinking in epoxidized natural rubber/dicarboxylic acid blends Polymer 2011 ;52: 5243-5249.

143 M. Pire, C. Lorthioir, E. K. Oikonomou, S. Norvez, I. lliopoulos, B. Le Rossignol and L. Leibler Imidazoleaccelerated crosslinking of epoxidized natural rubber by dicarboxylic acids: A mechanistic investigation using NMR spectroscopy Polym. Chem. 2012 ;3: 946-953.

144 D. Montarnal, F. Tournilhac, M. Hidalgo and L. Leibler Epoxy-based networks combining chemical and supramolecular hydrogen-bonding crosslinks J. Polym. Sci., Part A: Polym. Chem. 2010 ;48: 1133-1141.

145 C. E. Hoppe, M. J. Galante, P. A. Oyanguren and R. J. J. Williams Epoxies modified by palmitic acid: From hotmelt adhesives to plasticized networks Macromol. Mater. Eng. 2005 ;290: 456-462.

146 K. Dusek and L. Matejka Trans-esterification and gelation of polyhydroxy esters formed from diepoxides and dicarboxylic-acids Adv. Chem. Ser. 1984 ;208: 15-26.

147 J. E. White, J. D. Earls, J. W. Sherman, L. C. Lopez and M. L. Dettloff Step-growth polymerization of 10,11epoxyundecanoic acid. Synthesis and properties of a new hydroxy-functionalized thermoplastic polyester Polymer 2007 ;48: 3990-3998.

148 S. D. Lee, F. Sanda and T. Endo Thermal latency and structure-activity relationship of aminimides the polymerization of epoxide J. Polym. Sci., Part A: Polym. Chem. 1997 ;35: 689-694.

149 K. Suyama and M. Shirai Photobase generators: Recent progress and application trend in polymer systems Prog. Polym. Sci. 2009 ;34: 194-209.

150 F. Ricciardi, W. A. Romanchick and M. M. Joullie 1,3-dialkylimidazolium salts as latent catalysts in the curing of epoxy-resins J. Polym. Sci. Polym. Lett. Ed. 1983 ;21: 633-638.

151 S. P. Pappas, V. D. Kuntz and B. C. Pappas Latent amine catalysts for epoxy-carboxy hybrid powder coatings investigations on phase-change control of reactivity J. Coat. Technol. 1991 ;63: 39-46.

152 D. F. Parra, L. P. Mercuri, J. R. Matos, H. F. Brito and R. R. Romano Thermal behavior of the epoxy and polyester powder coatings using thermogravimetry/differential thermal analysis coupled gas chromatography/mass spectrometry (TG/DTA-GC/MS) technique: Identification of the degradation products Thermochim. Acta $2002 ; 386$ : 143-151.

153 D. Piazza, D. S. Silveira, N. P. Lorandi, E. J. Birriel, L. C. Scienza and A. J. Zattera Polyester-based powder coatings with montmorillonite nanoparticles applied on carbon steel Prog. Org. Coat. 2012 ;73: 42-46.

154 C. Ding and A. S. Matharu Recent developments on biobased curing agents: a review of their preparation and use ACS Sustainable Chem. Eng. 2014 ;2: 2217-2236. 
C. Ding, P. S. Shuttleworth, S. Makin, J. H. Clark and A. S. Matharu New insights into the curing of epoxidized linseed oil with dicarboxylic acids Green Chem. 2015 ;17: 4000-4008.

D. Guzman, X. Ramis, X. Fernandez-Francos and A. Serra New catalysts for diglycidyl ether of bisphenol A curing based on thiol-epoxy click reaction Eur. Polym. J. 2014 ;59: 377-386.

C. M. Seubert and M. E. Nichols Epoxy thiol photolatent base clearcoats: curing and formulation J. Coat. Technol. Res. 2010 ;7: 615-622.

D. Guzman, X. Ramis, X. Fernandez-Francos and A. Serra Enhancement in the glass transition temperature in latent thiol-epoxy click cured thermosets Polymers $2015 ; 7$ : 680-694.

R. Meizoso Loureiro, T. Carballeira Amarelo, S. Paz Abuin, E. R. Soule and R. J. J. Williams Kinetics of the epoxythiol click reaction initiated by a tertiary amine: Calorimetric study using monofunctional components Thermochim. Acta 2015 ;616: 79-86.

160 D. Caille, J. P. Pascault and L. Tighzert Reaction of a diepoxide with a disocyanate in bulk .1. Use of a tertiary amine catalyst Polym. Bull. $1990 ; 24:$ 23-30.

161 D. Caille, J. P. Pascault and L. Tighzert Reaction of a diepoxide with a diisocyanate in bulk .2. Use of an imidazole or a blocked isocyanate catalysts Polym. Bull. $1990 ; 24: 31-38$.

162 M. Flores, X. Fernandez-Francos, J. M. Morancho, A. Serra and X. Ramis Ytterbium triflate as a new catalyst on the curing of epoxy-isocyanate based thermosets Thermochim. Acta 2012 ;543: 188-196.

163 M. J. Galante and R. J. J. Williams Polymer networks based on the diepoxide diisocyanate reaction catalyzed by tertiary-amines J. Appl. Polym. Sci. 1995 ;55: 89-98.

164 Arundhati, R. Singhal and A. K. Nagpal Effect of polysulfide modifier on mechanical and morphological properties of epoxy/phthalic anhydride system Int. J. Plast. Tech. $2010 ; 13:$ 193-204.

165 G. B. Lowe The cure chemistry of polysulfides Int. J. Adhes. Adhes. 1997 ;17: 345-348.

166 H. Minami, M. Okubo and Y. Oshima Preparation of cured epoxy resin particles having one hollow by polyaddition reaction Polymer 2005 ;46: 1051-1056.

167 H. Minami, H. Kanamori, Y. Hata and M. Okubo Preparation of microcapsules containing a curing agent for epoxy resin by polyaddition reaction with the self-assembly of phase-separated polymer method in an aqueous dispersed system Langmuir 2008 ;24: 9254-9259.

168 D. Senatore, A. T. ten Cate, J. Laven, R. A. T. M. van Benthem and G. de With Temperature-triggered release of a liquid cross-linker micro-encapsulated in a glassy polymer for low temperature curing Polymer 2013 ;54: 75-83.

169 K. S. Toohey, N. R. Sottos, J. A. Lewis, J. S. Moore and S. R. White Self-healing materials with microvascular networks Nat. Mater. 2007 ;6: 581-585.

170 B. J. Blaiszik, S. L. B. Kramer, S. C. Olugebefola, J. S. Moore, N. R. Sottos and S. R. White Self-Healing Polymers and Composites Annu. Rev. Mater. Res. 2010 ;40: 179-211.

171 S. R. White, N. R. Sottos, P. H. Geubelle, J. S. Moore, M. R. Kessler, S. R. Sriram, E. N. Brown and S. Viswanathan Autonomic healing of polymer composites Nature 2001 ;409: 794-797.

172 D. A. Mcllroy, B. J. Blaiszik, M. M. Caruso, S. R. White, J. S. Moore and N. R. Sottos Microencapsulation of a reactive liquid-phase amine for self-healing epoxy composites Macromolecules 2010 ;43: 1855-1859.

173 P. W. Chen, G. Cadisch and A. R. Studart Encapsulation of aliphatic amines using microfluidics Langmuir 2014 ;30: 2346-2350.

174 H. Jin, C. L. Mangun, D. S. Stradley, J. S. Moore, N. R. Sottos and S. R. White Self-healing thermoset using encapsulated epoxy-amine healing chemistry Polymer 2012 ;53: 581-587. 
H. Jin, C. L. Mangun, A. S. Griffin, J. S. Moore, N. R. Sottos and S. R. White Thermally stable autonomic healing in epoxy using a dual-microcapsule system Adv. Mater. 2014 ;26: 282-287.

176 S. Neuser, P. W. Chen, A. R. Studart and V. Michaud Fracture toughness healing in epoxy containing both epoxy and amine loaded capsules Adv. Eng. Mater. 2014 ;16: 581-587.

177 Y. C. Yuan, M. Z. Rong, M. Q. Zhang, G. C. Yang and J. Q. Zhao Self-healing of fatigue crack in epoxy materials with epoxy/mercaptan system Express. Polym. Lett. $2011 ; 5:$ 47-59.

178 J. Yang, M. W. Keller, J. S. Moore, S. R. White and N. R. Sottos Microencapsulation of isocyanates for self-healing polymers Macromolecules 2008 ;41: 9650-9655.

179 A. P. Esser-Kahn, S. A. Odom, N. R. Sottos, S. R. White and J. S. Moore Triggered release from polymer capsules Macromolecules 2011 ; 44: 5539-5553.

180 D. A. Wicks and Z. W. Wicks Blocked isocyanates III Part B: Uses and applications of blocked isocyanates Prog. Org. Coat. $2001 ; 41: 1-83$.

181 D. A. Wicks and Z. W. Wicks Blocked isocyanates III: Part A. Mechanisms and chemistry Prog. Org. Coat. 1999 ;36: 148-172.

182 Z. W. Wicks New developments in the field of blocked isocyanates Prog. Org. Coat. $1981 ; 9: 3-28$.

183 R. Spindler and J. M. J. Frechet Synthesis and characterization of hyperbranched polyurethanes prepared from blocked isocyanate monomers by step-growth polymerization Macromolecules 1993 ;26: 4809-4813.

184 K. Suzuki, N. Matsu-ura, H. Horii, Y. Sugita, F. Sanda and T. Endo Diethyl ketone-based imine as efficient latent hardener for epoxy resin J. Appl. Polym. Sci. 2002 ;83: 1744-1749.

185 K. Suzuki, N. Matsu-Ura, H. Horii, Y. Sugita, F. Sanda and T. Endo Reaction of glycidyl phenyl ether with imines: A model study of latent hardeners of epoxy resins in the presence of water J. Polym. Sci., Part A: Polym. Chem.

2002 ; 40: 971-975.

186 K. Suzuki, H. Horii, Y. Sugita, F. Sanda and T. Endo Promotion effect of bifunctional five-membered cyclic dithiocarbonate on curing of one-component epoxy resin by imines as latent initiator J. Appl. Polym. Sci. 2004 ;94: 961-964.

187 K. Suzuki, H. Horii, Y. Sugita, F. Sanda and T. Endo Role of cyclic dithiocarbonate as a promoter in the reaction of epoxide and imine in the presence of water J. Polym. Sci., Part A: Polym. Chem. 2004 ;42: 4276-4283.

188 K. Suzuki, Y. Sugita, F. Sanda and T. Endo One-component epoxy resin with imine as water-initiated latent hardener: Improvement of the mechanical and adhesive properties by the addition of methacrylate copolymer $\mathrm{J}$. Appl. Polym. Sci. 2005 ;96: 1943-1949.

189 H. Okuhira, T. Kii, M. Ochi and H. Takeyama Novel moisture-curable epoxy resins and their characterization J. Appl. Polym. Sci. 2003 ;89: 91-95.

190 H. Okuhira, T. Kii, M. Ochi and H. Takeyama Characterization of epoxy resin hardening with ketimine latent hardeners J. Adhes. Sci. Technol. 2004 ;18: 205-211.

191 H. Okuhira, N. Iwamoto, M. Ochi and H. Takeyama Novel urethane/epoxy resin hybrid materials using a moisturecuring system J. Polym. Sci., Part B: Polym. Phys. 2004 ;42: 1137-1144.

192 J. E. Beecher, J. F. Cameron and J. M. J. Frechet Photogeneration of reactive amine functionalities within polymers - synthesis of photoprecursor copolymers and their use in photocuring Abstr. Pap. Am. Chem. Soc. 1991 ;201: 40-PMSE.

193 J. F. Cameron and J. M. J. Frechet Photogeneration of organic-bases from ortho-nitrobenzyl-derived carbamates J. Am. Chem. Soc. 1991 ;113: 4303-4313. 
J. F. Cameron and J. M. J. Frechet Solid-state quantum yield determination of a novel base photogenerator J. Photochem. Photobiol. A Chem. 1991 ;59: 105-113.

195 J. M. J. Frechet and J. F. Cameron Photogenerated amines and diamines - novel curing systems for thin-film coatings Abstr. Pap. Am. Chem. Soc. 1991 ;201: 31-PMSE.

196 K. Arimitsu, M. Miyamoto and K. Ichimura Applications of a nonlinear organic reaction of carbamates to proliferate aliphatic amines Angew. Chem. Int. Ed. Engl. 2000 ;39: 3425-3428.

197 K. Ito, M. Nishimura, M. Sashio and M. Tsunooka Photo-initiated amine-formation in a polymer matrix and its application to thermal cross-linking of poly(glycidyl methacrylate) films Chem. Lett. 1992 1153-1156.

198 K. I. Ito, M. Nishimura, M. Sashio and M. Stunooka Photo-initiated base-formation in a polymer matrix J. Polym. Sci., Part A: Polym. Chem. 1994 ;32: 2177-2185.

199 K. I. Ito, M. Nishimura, M. Sashio and M. Tsunooka Thermal cross-linking of poly(glycidyl methacrylate) films and epoxy-resin films using amines formed by photolysis of o-acyloximes J. Polym. Sci., Part A: Polym. Chem. 1994 ;32: 1793-1796.

200 K. I. Ito, Y. Shigeru, Y. U. Kawata, K. Ito and M. Tsunooka Photo-initiated and thermal curing of epoxides by the use of photo base generators bearing acyloxyimino groups Can. J. Chem. 1995 ;73: 1924-1932.

201 K. Suyama, S. Ozaki and M. Shirai Photo-crosslinking of polymeric photobase generator bearing O-acyloxime moieties with low eliminating by-products and high sensitivity React. Funct. Polym. 2013 ;73: 518-523.

202 T. Ohba, D. Nakai, K. Suyama and M. Shirai Photo-crosslinking of poly(glycidyl methacrylate) using di-functional photobase generators J. Photopolym. Sci. Technol. 2004 ;17: 11-14.

203 J. Lalevee, X. Allonas, J. P. Fouassier, H. Tachi, A. Izumitani, M. Shirai and M. Tsunooka Investigation of the photochemical properties of an important class of photobase generators: the O-acyloximes J. Photochem. Photobiol. A Chem. 2002 ;151: 27-37.

204 X. D. Liu, M. Kimura, A. Sudo and T. Endo Accelerating effects of N-aryl-N',N'-dialkyl ureas on epoxydicyandiamide curing system J. Polym. Sci., Part A: Polym. Chem. 2010 ;48: 5298-5305.

205 N. Poisson, A. Maazouz, H. Sautereau, M. Taha and X. Gambert Curing of dicyandiamide epoxy resins accelerated with substituted ureas J. Appl. Polym. Sci. 1998 ;69: 2487-2497.

206 D. Guzman, X. Ramis, X. Fernandez-Francos and A. Serra Preparation of click thiol-ene/thiol-epoxy thermosets by controlled photo/thermal dual curing sequence RSC Adv. 2015 ;5: 101623-101633.

207 H. Komatsu, T. Hino and T. Endo Thermal dissociation behavior of copolymers bearing hemiacetal ester moieties and their reactions with epoxides J. Polym. Sci., Part A: Polym. Chem. 2006 ;44: 3966-3977.

208 H. Komatsu, B. Ochiai, T. Hino and T. Endo Model reaction for thermally latent curing through addition of hemiacetal ester and epoxide by Schiff-base-zinc halide complexes J. Polym. Sci., Part A: Polym. Chem. 2007 ;45: 3370-3379.

209 H. Komatsu, B. Ochiai, T. Hino and T. Endo Thermally latent reaction of hemiacetal ester with epoxide controlled by Schiff-base-zinc chloride complexes with tunable catalytic activity J. Mol. Catal. A Chem. 2007 ;273: 289-297.

210 S. Saito, K. Nakasato, Y. Katoh, Y. Oshibe and M. Ishidoya Application of thermal latent carboxylic acid derivatives to Pb-free microsoldering Mater. Trans. 2004 ;45: 759-764.

211 T. Yamamoto and M. Ishidoya New thermosetting coatings using blocked carboxyl groups Prog. Org. Coat. 2000 ;40: 267-273.

212 Y. Nakane and M. Ishidoya New crosslinking system using blocked carboxylic acid Prog. Org. Coat. 1997 ;31: $113-$ 120. 

of epoxy-resins using the thiol Polym. J. 1994 ;26: 864-867.

214 T. Nishikubo, A. Kameyama, K. Kashiwagi and N. Oyama Novel thermal curing reactions of epoxy resin and poly(glycidyl methacrylate) using photo-generated difunctional thiols Polym. J. 1996 ;28: 795-800.

215 H. Otsuka and T. Endo Poly(hemiacetal ester)s: New class of polymers with thermally dissociative units in the main chain Macromolecules 1999 ;32: 9059-9061.

216 H. Otsuka, H. Fujiwara and T. Endo Fine-tuning of thermal dissociation temperature using copolymers with hemiacetal ester moieties in the side chain: Effect of comonomer on dissociation temperature React. Funct. Polym. 2001 ;46: 293-298.

217 N. N. Ghosh and G. R. Palmese Electron-beam curing of epoxy resins: effect of alcohols on cationic polymerization Bull. Mater. Sci. 2005 ;28: 603-607.

218 M. Mascioni, N. N. Ghosh, J. M. Sands and G. R. Palmese Electron beam and UV cationic polymerization of glycidyl ethers - PART I: Reaction of monofunctional phenyl glycidyl ether J. Appl. Polym. Sci. 2013 ;130: 479-486.

219 M. Mascioni, N. N. Ghosh, J. M. Sands and G. R. Palmese Electron beam and UV cationic polymerization of glycidyl ethers PART II: Reaction of diglycidyl ether of bisphenol A J. Appl. Polym. Sci. 2013 ;130: 487-495.

220 M. S. Heise and G. C. Martin Analysis of the cure kinetics of epoxy imidazole resin systems J. Appl. Polym. Sci. $1990 ; 39: 721-738$.

221 J. M. Barton, G. J. Buist, I. Hamerton, B. J. Howlin, J. R. Jones and S. U. Liu Preparation and characterization of imidazole-metal complexes and evaluation of cured epoxy networks J. Mater. Chem. 1994 ;4: 379-384. J. M. Barton, I. Hamerton, B. J. Howlin, J. R. Jones and S. Y. Liu Studies of cure schedule and final property relationships of a commercial epoxy resin using modified imidazole curing agents Polymer 1998 ;39: 1929-1937. I. Hamerton, A. M. Emsley, J. N. Hay, H. Herman, B. J. Howlin and P. Jepson The development of controllable complex curing agents for epoxy resins - Part 3. An investigation of the shelf life and thermal dissociation behaviour of bis(acetanilido)tris(acetato)dicuprate(II) J. Mater. Chem. 2006 ;16: 255-265.

224 M. A. M. Rahmathullah, A. Jeyarajasingam, B. Merritt, M. VanLandingham, S. H. McKnight and G. R. Palmese Room temperature ionic liquids as thermally latent initiators for polymerization of epoxy resins Macromolecules 2009 ;42: 3219-3221. K. Kowalczyk and T. Spychaj lonic liquids as convenient latent hardeners of epoxy resins Polimery $2003 ; 48: 833-$ 835.

H. Maka, T. Spychaj and R. Pilawka Epoxy resin/ionic liquid systems: The influence of imidazolium cation size and anion type on reactivity and thermomechanical properties Ind. Eng. Chem. Res. $2012 ; 51:$ 5197-5206. J. A. Throckmorton, A. L. Watters, X. Geng and G. R. Palmese Room temperature ionic liquids for epoxy nanocomposite synthesis: Direct dispersion and cure Compos. Sci. Technol. 2013 ;86: 38-44.

228 A. A. Silva, S. Livi, D. B. Netto, B. G. Soares, J. Duchet and J.-F. Gerard New epoxy systems based on ionic liquid Polymer 2013 ;54: 2123-2129.

229 B. Guenther Soares, S. Livi, J. Duchet-Rumeau and J. F. Gerard Preparation of epoxy/MCDEA networks modified with ionic liquids Polymer 2012 ;53: 60-66.

230 B. G. Soares, S. Livi, J. Duchet-Rumeau and J.-F. Gerard Synthesis and characterization of epoxy/MCDEA networks modified with imidazolium-based ionic liquids Macromol. Mater. Eng. 2011 ;296: 826-834.

231 M. J. Shin, Y. J. Shin, S. W. Hwang and J. S. Shin Microencapsulation of imidazole curing agent by solvent evaporation method using W/O/W emulsion J. Appl. Polym. Sci. $2013 ; 129$ : 1036-1044. 

for epoxy resin J. Ind. Eng. Chem. 2010 ;16: 728-733.

233 M. J. Shin, J. G. Kim and J. S. Shin Microencapsulation of imidazole curing agents by spray-drying method using W/O emulsion J. Appl. Polym. Sci. 2012 ;126: E108-E115.

234 D. H. Lee, M. Yang, S. H. Kim, M. J. Shin and J. S. Shin Microencapsulation of imidazole curing agents by spraydrying method J. Appl. Polym. Sci. $2011 ; 122:$ 782-788.

235 N. Makiuchi, A. Sudo and T. Endo Ring opening polymerization of epoxides with urea-derivatives of 4aminopyridine as thermally latent anionic initiator J. Polym. Sci., Part A: Polym. Chem. 2014 ;52: 2518-2522.

236 M. Kim, F. Sanda and T. Endo Phosphonamidates as thermally latent anionic initiators of glycidyl phenyl ether: Substituent effect on the initiator activity Macromolecules $2001 ; 34:$ 409-414.

237 M. Kim, F. Sanda and T. Endo Phosphonamidates as thermally latent initiators in the polymerization of epoxides Polym. Bull. $\quad 2001 ; 46: 277-283$.

238 M. Kim, F. Sanda and T. Endo Phosphonamidodithioate as a novel thermally latent initiator in the polymerization of glycidyl phenyl ether Tetrahedron Lett. $2000 ; 41:$ 7487-7491.

239 M. Kim, F. Sanda and T. Endo Polymerization of glycidyl phenyl ether with phosphonic amide ester as a novel thermally latent anionic initiator Macromolecules 2000 ;33: 3499-3501.

240 M. Kirino and I. Tomita Aminimides derived from benzoylformic acid esters as thermally latent base catalysts Macromolecules 2010 ;43: 8821-8827.

241 M. Kirino and I. Tomita Aminimides derived from p-substituted benzoylformic acid ester as thermal/photolatent bases and photoradical initiators J. Polym. Sci., Part A: Polym. Chem. 2013 ;51: 4292-4300.

242 K. Dietliker, R. Huesler, J. L. Birbaum, S. Ilg, S. Villeneuve, K. Studer, T. Jung, J. Benkhoff, H. Kura, A. Matsumoto and H. Oka Advancements in photoinitiators - Opening up new applications for radiation curing Prog. Org. Coat. 2007 ;58: 146-157.

$243 \quad$ X. Y. Yu, J. Chen, J. W. Yang, Z. H. Zeng and Y. L. Chen Preparation of a series of photoinitiators and their use in the thermal curing of epoxide and radical polymerization of acrylate Polymer $2005 ; 46$ : 5736-5745.

244 A. Chemtob, F. Courtecuisse, C. Croutxe-Barghorn and S. Rigolet Simultaneous sol-gel and anionic photopolymerization of 3-(glycidyloxypropyl)trimethoxysilane via photobase catalysis New J. Chem. $2011 ; 35$ : 1803-1808.

245 H. Salmi, X. Allonas, C. Ley, D. Marechal and A. Ak Photopolymerization using photolatent amine catalysts J. Photopolym. Sci. Technol. 2012 ;25: 147-151.

246 P. Bruzga, J. V. Grazulevicius and R. Kavaliunas Cationic polymerization of 1,2-epoxy-6-(9-carbazolyl)-4oxahexane Eur. Polym. J. 1991 ;27: 707-711.

247 C. C. Price and M. Osgan The polymerization of I-propylene oxide J. Am. Chem. Soc. 1956 ;78: 4787-4792.

248 R. O. Colclough, G. Gee, W. C. E. Higginson, J. B. Jackson and M. Litt The polymerization of epoxides by metal halide catalysts J. Polym. Sci., Part A: Polym. Chem. 1959 ;34: 171-179.

249 M. I. Childers, J. M. Longo, N. J. Van Zee, A. M. LaPointe and G. W. Coates Stereoselective epoxide polymerization and copolymerization Chem. Rev. $2014 ; 114:$ : 8129-8152.

250 J. Atchekzai, B. Bonnetot, B. France, H. Mongeot, A. Anton, A. Dubuisson and P. Chastagner Catalytic curing agents - a study of boron halide and boron isothiocyanate complexes with tertiary-amines as catalytic curing agents for epoxide and epoxy-isocyanate mixtures Polym. Bull.

$1991 ; 27: 47-52$. 
B. Bonnetot, H. Mongeot and V. Razafindrakoto Catalytic curing agents - Decomposition of trihalo and triisothiocyanato borane adducts with tertiary amines in curing epoxy resins: Towards an understanding of the mechanism of polymerization Polym. Bull. 1996 ;37: 665-662.

252 S. J. Garcia, A. Serra and J. Suay Lanthanide triflates as curing initiators for solid DGEBA resin: Thermal and mechanical characterization J. Appl. Polym. Sci. 2007 ;105: 3097-3107.

253 S. J. Garcia, X. Ramis, A. Serra and J. Suay Cationic crosslinking of solid DGEBA resins with ytterbium(III) trifluoromethanesulfonate as initiator J. Therm. Anal. Calorim. 2006 ;83: 429-438.

254 S. J. Garcia, X. Ramis, A. Serra and J. Suay Addition effect of erbium(III) trifluoromethanesulfonate in the homopolymerization kinetics of a DGEBA resin Thermochim. Acta 2006 ;441: 45-52.

255 P. Castell, M. Galia, A. Serra, J. M. Salla and X. Ramis Study of lanthanide triflates as new curing initiators for DGEBA Polymer 2000 ;41: 8465-8474.

256 D. S. Xiao, Y. C. Yuan, M. Z. Rong and M. Q. Zhang A facile strategy for preparing self-healing polymer composites by incorporation of cationic catalyst-loaded vegetable fibers Adv. Funct. Mater. 2009 ;19: 2289-2296.

257 A. J. Ryan, U. R. Vaidya, W. Mormann and C. W. Macosko Networks by fast epoxy polymerization Polym. Bull. 1990 ;24: 521-527.

258 P. Chabanne, L. Tighzert, J. P. Pascault and B. Bonnetot Epoxy polymerization initiated by BF3-amine complexes .1. Synthesis and characterization of BF3-amine complexes and BF3-amine complexes dissolved in gammabutyrolactone J. Appl. Polym. Sci. 1993 ;49: 685-699.

259 P. Chabanne, L. Tighzert and J. P. Pascault Monoepoxy polymerization initiated by BF3-amine complexes in bulk .3. Influence of gamma-butyrolactone on polymer formation J. Appl. Polym. Sci. 1994 ;53: 787-806.

260 J. J. Harris and S. C. Temin Proposed mechanism for curing of epoxy resins with amine-lewis acid complexes or salts J. Appl. Polym. Sci. $1966 ; 10:$ 523-534.

261 N. Bouillon, J. P. Pascault and L. Tighzert Epoxy prepolymers cured with boron trifluoride-amine complexes .2. Polymerization mechanisms Makromol. Chem. Macromol. Chem. Phys. 1990 ;191: 1417-1433.

262 N. Bouillon, J. P. Pascault and L. Tighzert Epoxy prepolymers cured with boron trifluoride-amine complexes .3. Polymerization mechanism of a prepolymer containing epoxy groups Makromol. Chem. Macromol. Chem. Phys. 1990 ;191: 1435-1449.

263 M. Akatsuka, Y. Takezawa and S. Amagi Influences of inorganic fillers on curing reactions of epoxy resins initiated with a boron trifluoride amine complex Polymer 2001 ;42: 3003-3007.

264 M. Tackie and G. C. Martin The polymerization mechanism and kinetics of DGEBA with BF3-MEA J. Appl. Polym. Sci. 1993 ;48: 793-808.

265 M. Ghaemy and H. Khandani Kinetics of curing reaction of DGEBA with BF3-amine complexes using isothermal DSC technique Eur. Polym. J. 1998 ;34: 477-486.

266 M. Ghaemy The polymerization mechanism and kinetics of DGEBA with BF3-EDA Eur. Polym. J. 1998 ;34: 11511156.

267 R. E. Smith, F. N. Larsen and C. L. Long Epoxy-resin cure .1. F-19 NMR of boron-trifluoride monoethylamine and fluoroboric acid J. Appl. Polym. Sci. 1984 ;29: 3697-3711.

268 R. E. Smith, F. N. Larsen and C. L. Long Epoxy-resin cure .2. FTIR analysis J. Appl. Polym. Sci. 1984 ;29: 37133726 . 

accelerator for prolonging the shelf-life of epoxy resin-based microelectronic packaging material J. Mater. Chem. 2002 ; $12: 2325-2330$.

270 D. S. Xiao, Y. C. Yuan, M. Z. Rong and M. Q. Zhang Hollow polymeric microcapsules: Preparation, characterization and application in holding boron trifluoride diethyl etherate Polymer 2009 ;50: 560-568.

271 D. S. Xiao, Y. C. Yuan, M. Z. Rong and M. Q. Zhang Self-healing epoxy based on cationic chain polymerization Polymer 2009 ;50: 2967-2975.

272 J. V. Crivello and J. H. W. Lam New preparation of triarylsulfonium and triarylselenonium salts via copper(II)catalyzed arylation of sulfides and selenides with diaryliodonium salts J. Org. Chem. 1978 ;43: 3055-3058.

273 J. V. Crivello and J. H. W. Lam New route to triarylsulfonium salts via the double arylation of thiophenols Synth. Commun. 1979 ;9: 151-156.

274 J. V. Crivello and J. H. W. Lam Photosensitive polymers containing diaryliodonium salt groups in the main chain J. Polym. Sci., Part A: Polym. Chem. 1979 ;17: 3845-3858.

275 J. V. Crivello and J. H. W. Lam Triarylsulfonium salts as photoinitiators of free-radical and cationic polymerization J. Polym. Sci. Polym. Lett. Ed. $1979 ; 17:$ 759-764.

276 J. V. Crivello The discovery and development of onium salt cationic photoinitiators J. Polym. Sci., Part A: Polym. Chem. 1999 ;37: 4241-4254.

277 Y. Yagci, S. Jockusch and N. J. Turro Photoinitiated Polymerization: Advances, Challenges, and Opportunities Macromolecules 2010 ;43: 6245-6260.

278 M. Sangermano, N. Razza and J. V. Crivello Cationic UV-curing: technology and applications Macromol. Mater. Eng. 2014 ;299: 775-793.

279 Y. Yagci, Y. Y. Durmaz and B. Aydogan Phenacyl onium salt photoinitiators: synthesis, photolysis, and applications Chem. Rec. 2007 ; 7: 78-90.

280 U. Bulut and J. V. Crivello Investigation of the reactivity of epoxide monomers in photoinitiated cationic polymerization Macromolecules 2005 ;38: 3584-3595.

281 J. V. Crivello Cationic photopolymerization of alkyl glycidyl ethers J. Polym. Sci., Part A: Polym. Chem. 2006 ;44: 3036-3052.

282 J. V. Crivello Design and synthesis of multifunctional glycidyl ethers that undergo frontal polymerization J. Polym. Sci., Part A: Polym. Chem. 2006 ;44: 6435-6448.

283 C. Y. Ryu, M. J. Spencer and J. V. Crivello Involvement of supramolecular complexes in the capture and release of protonic acids during the cationic ring-opening polymerization of epoxides Macromolecules 2012 ;45: 2233-2241.

284 J. V. Crivello and R. Narayan Epoxidized triglycerides as renewable monomers in photoinitiated cationic polymerization Chem. Mater. 1992 ;4: 692-699.

285 J. V. Crivello and S. S. Liu Photoinitiated cationic polymerization of epoxy alcohol monomers J. Polym. Sci., Part A: Polym. Chem. 2000 ;38: 389-401.

286 M. J. M. Abadie, N. K. Chia and F. Boey Cure kinetics for the ultraviolet cationic polymerization of cycloliphatic and diglycidyl ether of bisphenol-A (DGEBA) epoxy systems with sulfonium salt using an auto catalytic model J. Appl. Polym. Sci. 2002 ;86: 1587-1591.

287 A. Hartwig, K. Koschek, A. Luhring and O. Schorsch Cationic polymerization of a cycloaliphatic diepoxide with latent initiators in the presence of structurally different diols Polymer $2003 ; 44: 2853-2858$. 

epoxidized rubber Eur. Polym. J. 1996 ;32: 1319-1331.

289 M. Sangermano, M. A. Tasdelen and Y. Yagci Photoinitiated curing of mono- and bifunctional epoxides by combination of active chain end and activated monomer cationic polymerization methods J. Polym. Sci., Part A: Polym. Chem. 2007 ;45: 4914-4920.

290 E. Takahashi, F. Sanda and T. Endo Photocationic and radical polymerizations of epoxides and acrylates by novel sulfonium salts J. Polym. Sci., Part A: Polym. Chem. 2003 ;41: 3816-3827.

291 T. Takata, K. Takuma and T. Endo Photoinitiated cationic polymerization of epoxide with phosphonium salts as novel photolatent initiators Makromol. Chem. Rapid Commun. 1993 ;14: 203-206.

292 J. Ammer, C. F. Sailer, E. Riedle and H. Mayr Photolytic generation of benzhydryl cations and radicals from quaternary phosphonium salts: how highly reactive carbocations survive their first nanoseconds J. Am. Chem. Soc. 2012 ; 134: 11481-11494.

293 J. Ammer and H. Mayr Photogeneration of carbocations: applications in physical organic chemistry and the design of suitable precursors J. Phys. Org. Chem. 2013 ;26: 956-969.

294 I. Abu-Abdoun and Aale-Ali Cationic photopolymerization of cyclohexene oxide Eur. Polym. J. 1992 ;28: 73-78.

295 L. Atmaca, I. Kayihan and Y. Yagci Photochemically and thermally induced radical promoted cationic polymerization using allyl phosphonium salts Polymer $2000 ; 41$ : 6035-6041.

296 Y. Yagci and T. Endo N-benzyl and N-alkoxy pyridinium salts as thermal and photochemical initiators for cationic polymerization Adv. Polym. Sci. 1997 ;127: 59-86.

297 N. Yonet, N. Bicak and Y. Yagci Photoinitiated cationic polymerization of cyclohexene oxide by using phenacyl benzoylpyridinium salts Macromolecules 2006 ;39: 2736-2738.

298 E. Takahashi, F. Sanda and T. Endo Novel pyridinium salts as cationic thermal and photoinitiators and their photosensitization properties J. Polym. Sci., Part A: Polym. Chem. 2002 ;40: 1037-1046.

299 Y. Yagci, I. Kminek and W. Schnabel Photochemical cationic polymerization of cyclohexene oxide in solution containing pyridinium salt and polysilane Eur. Polym. J. 1992 ;28: 387-390.

300 Y. Yagci, A. Kornowski and W. Schnabel $\mathrm{N}$-alkoxy-pyridinium and $\mathrm{N}$-alkoxy-quinolinium salts as initiators for cationic photopolymerizations J. Polym. Sci., Part A: Polym. Chem. 1992 ;30: 1987-1991.

301 Y. Yagci, I. Lukac and W. Schnabel Photosensitized cationic polymerization using n-ethoxy-2-methylpyridinium hexafluorophosphate Polymer 1993 ;34: 1130-1133.

302 D. Dossow, Q. Q. Zhu, G. Hizal, Y. Yagci and W. Schnabel Photosensitized cationic polymerization of cyclohexene oxide: A mechanistic study concerning the use of pyridinium-type salts Polymer 1996 ;37: 2821-2826.

303 F. Kasapoglu, A. Onen, N. Bicak and Y. Yagci Photoinitiated cationic polymerization using a novel phenacyl anilinium salt Polymer 2002 ;43: 2575-2579.

304 F. Kasapoglu and Y. Yagci Photo sensitized cationic polymerization of cyclohexene oxide using a phenacylanilinium salt Macromol. Rapid Commun. 2002 ;23: 567-570.

305 J. V. Crivello and S. Q. Kong Photoinduced and thermally induced cationic polymerizations using dialkylphenacylsulfonium salts Macromolecules $2000 ; 33: 833-842$.

306 J. V. Crivello and S. Q. Kong Synthesis and characterization of second-generation dialkylphenacylsulfonium salt photoinitiators Macromolecules 2000 ;33: 825-832.

307 J. Shao, Y. Huang and Q. Fan Visible light initiating systems for photopolymerization: status, development and challenges Polym. Chem. 2014 ;5: 4195-4210. 

Visible light sensitive photoinitiating systems: Recent progress in cationic and radical photopolymerization reactions under soft conditions Prog. Polym. Sci. 2015 ;41: 32-66.

309 J. P. Fouassier, D. Burr and J. V. Crivello Photochemistry and photopolymerization activity of diaryliodonium salts J. Macromol. Sci., Part A: Pure Appl. Chem. 1994 ;31: 677-701.

310 M. Jin, H. Hong, J. Xie, J.-P. Malval, A. Spangenberg, O. Soppera, D. Wan, H. Pu, D.-L. Versace, T. Leclerc, P. Baldeck, O. Poizat and S. Knopf pi-conjugated sulfonium-based photoacid generators: An integrated molecular approach for efficient one and two-photon polymerization Polym. Chem. 2014 ;5: 4747-4755.

311 G. Yilmaz, S. Beyazit and Y. Yagci Visible light induced free radical promoted cationic polymerization using thioxanthone derivatives J. Polym. Sci., Part A: Polym. Chem. 2011 ;49: 1591-1596.

312 F. A. M. Abdulrasoul, A. Ledwith and Y. Yagci Thermal and photo-chemical cationic polymerizations induced by AgPF6 in presence of free-radical initiators Polym. Bull. 1978 ;1: 1-6.

313 Y. Y. Durmaz, N. Moszner and Y. Yagci Visible light initiated free radical promoted cationic polymerization using acylgermane based photoinitiator in the presence of onium salts Macromolecules 2008 ;41: 6714-6718.

314 J. V. Crivello and U. Bulut Indian turmeric and its use in cationic photopolymerizations Macromol. Symp. 2006 ;240: 1-11.

315 G. Hizal, Y. Yagci and W. Schnabel Charge-transfer complexes of pyridinium ions and methyl-substituted and methoxy-substituted benzenes as photoinitiators for the cationic polymerization of cyclohexene oxide and relatedcompounds Polymer 1994 ;35: 2428-2431.

316 B. Aydogan, Y. Yagci, L. Toppare, S. Jockusch and N. J. Turro Photoinduced Electron Transfer Reactions of Highly Conjugated Thiophenes for Initiation of Cationic Polymerization and Conjugated Polymer Formation Macromolecules 2012 ;45: 7829-7834.

317 J. V. Crivello and U. Bulut Curcumin: A naturally occurring long-wavelength photosensitizer for diaryliodonium salts J. Polym. Sci., Part A: Polym. Chem. 2005 ;43: 5217-5231.

318 J. Lalevee, N. Blanchard, M.-A. Tehfe, F. Morlet-Savary and J. P. Fouassier Green bulb light source induced epoxy cationic polymerization under air using tris(2,2'-bipyridine)ruthenium(II) and silyl radicals Macromolecules $2010 ; 43$ : 10191-10195.

319 M.-A. Tehfe, J. Lalevee, D. Gigmes and J. P. Fouassier Green chemistry: Sunlight-induced cationic polymerization of renewable epoxy monomers under air Macromolecules 2010 ;43: 1364-1370.

320 J. Lalevee and J. P. Fouassier Recent advances in sunlight induced polymerization: Role of new photoinitiating systems based on the silyl radical chemistry Polym. Chem. $2011 ; 2:$ 1107-1113.

321 P. Xiao, F. Dumur, B. Graff, J. P. Fouassier, D. Gigmes and J. Lalevee Cationic and thiol-ene photopolymerization upon red lights using anthraquinone derivatives as photoinitiators Macromolecules $2013 ; 46$ : 6744-6750.

322 P. Xiao, F. Dumur, D. Thirion, S. Fagour, A. Vacher, X. Sallenave, F. Morlet-Savary, B. Graff, J. P. Fouassier, D. Gigmes and J. Lalevee Multicolor photoinitiators for radical and cationic polymerization: Monofunctional vs polyfunctional thiophene derivatives Macromolecules 2013 ;46: 6786-6793.

323 P. Xiao, F. Dumur, B. Graff, D. Gigmes, J. P. Fouassier and J. Lalevee Blue light sensitive dyes for various photopolymerization reactions: Naphthalimide and naphthalic anhydride derivatives Macromolecules $2014 ; 47$ : 601-608. 
P. Xiao, M. Frigoli, F. Dumur, B. Graff, D. Gigmes, J. P. Fouassier and J. Lalevee Julolidine or fluorenone based push-pull dyes for polymerization upon soft polychromatic visible light or green light Macromolecules $2014 ; 47$ : 106-112.

J. Zhang, M. Frigoli, F. Dumur, P. Xiao, L. Ronchi, B. Graff, F. Morlet-Savary, J. P. Fouassier, D. Gigmes and J. Lalevee Design of Novel Photoinitiators for Radical and Cationic Photopolymerizations under Near UV and Visible LEDs (385, 395, and 405 nm) Macromolecules 2014 ;47: 2811-2819.

J. Zhang, F. Dumur, P. Xiao, B. Graff, D. Bardelang, D. Gigmes, J. P. Fouassier and J. Lalevee Structure design of naphthalimide derivatives: Toward versatile photoinitiators for near-uvnisible leds, 3D printing, and water-soluble photoinitiating systems Macromolecules 2015 ;48: 2054-2063.

S. P. Pappas and L. W. Hill Kinetic parameter considerations for maximizing stability and minimizing cure temperature of thermosetting coatings - sulfonium salts as latent thermal initiators for cationic polymerization $\mathrm{J}$. Coat. Technol. $1981 ; 53: 43-51$.

328 K. Morio, H. Murase, H. Tsuchiya and T. Endo Thermoinitiated cationic polymerization of epoxy-resins by sulfonium salts J. Appl. Polym. Sci. 1986 ;32: 5727-5732.

F. Hamazu, S. Akashi, T. Koizumi, T. Takata and T. Endo Novel benzyl sulfonium salt having an aromatic group on sulfur atom as a latent thermal initiator J. Polym. Sci., Part A: Polym. Chem. 1991 ;29: 1675-1680.

F. Hamazu, S. Akashi, T. Koizumi, T. Takata and T. Endo Synthesis and activity of aryl benzyl methyl sulfonium salts as cationic initiators - effect of substituent on aryl group J. Polym. Sci., Part A: Polym. Chem. 1991 ;29: 18451851.

331 F. Hamazu, S. Akashi, T. Koizumi, T. Takata and T. Endo Cationic polymerization with p-substituted benzyl phydroxyphenyl methyl sulfonium salts - effect of substituents and mechanistic aspects of initiation reaction $\mathrm{J}$. Polym. Sci., Part A: Polym. Chem. 1993 ;31: 1023-1028.

332 O. Shimomura, I. Tomita and T. Endo Cationic polymerization of glycidyl phenyl ether initiated by various arylsulfonium salts as new thermal latent cationic initiators Macromol. Rapid Commun. 1998 ;19: 493-497.

O. Shimomura, I. Tomita and T. Endo Cationic polymerization of glycidyl phenyl ether initiated by sulfonium salts of phenoxathiin and thianthrene as novel thermal latent cationic initiators J. Polym. Sci., Part A: Polym. Chem. 1999 ;37: 127-128.

334 O. Shimomura, I. Tomita and T. Endo Application of S-alkylsulfonium salts of aromatic sulfides as new thermal latent cationic initiators J. Polym. Sci., Part A: Polym. Chem. 2000 ;38: 18-27.

335 O. Shimomura, I. Tomita and T. Endo Curing behavior of epoxy resin initiated by S-alkylsulfonium salts of aromatic sulfides as thermal latent cationic initiators J. Polym. Sci., Part A: Polym. Chem. 2001 ;39: 868-871.

336 E. Takahashi, F. Sanda and T. Endo Novel sulfonium salts as thermal and photoinitiators for epoxide and acrylate polymerizations J. Appl. Polym. Sci. 2004 ;91: 589-597.

337 T. Toneri, F. Sanda and T. Endo Polymerization of epoxide by a fluorenyltriphenylphosphonium salt as a thermally latent initiator J. Polym. Sci., Part A: Polym. Chem. 1998 ;36: 1957-1960.

338 T. Toneri, F. Sanda and T. Endo Cationic polymerization of epoxide by fluorenylphosphonium salts as thermally latent initiators. Substituent effect on the initiator activity Macromolecules 2001 ;34: 1518-1521.

339 M. Kobayashi, F. Sanda and T. Endo Application of phosphonium ylides to latent catalysts for polyaddition of bisphenol A diglycidyl ether with bisphenol A: Model system of epoxy-novolac resin Macromolecules 1999 ;32: 4751-4756. 
M. Kobayashi, F. Sanda and T. Endo Application of phosphonium ylides to latent catalysts. 2. Kinetic study on the thermal latency of the phosphonium ylides in the polyaddition of bisphenol A diglycidyl ether with bisphenol A Macromolecules 2000 ;33: 5384-5387.

341 S. Nakano and T. Endo Thermal cationic curing by benzylpyridinium salts Prog. Org. Coat. $1994 ; 23:$ 379-385.

342 S. B. Lee, T. Takata and T. Endo (Para-methoxybenzyl)-p-cyanopyridinium and (alpha-methylbenzyl)-pcyanopyridinium hexafluoroantimonates - activated latent thermal catalysts Macromolecules 1990 ;23: 431-434.

343 S. B. Lee, T. Takata and T. Endo Quaternary ammonium-salts as useful cationic initiators .6. Synthesis, activity, and thermal latency of n-benzylpyridinium salts and the role of the pyridine moiety Macromolecules $1991 ; 24$ : 2689-2693.

344 S. Nakano and T. Endo The effect of substituents on the catalytic activity of benzylpyridinium salts and their application to non-solvent type thermosetting compositions Polimery 1998 ;43: 679-684.

345 S. Nakano and T. Endo Thermal cationic curing with benzylammonium salts Prog. Org. Coat. $1993 ; 22: 287-300$.

346 S. Nakano and T. Endo Cationic polymerization of glycidyl phenyl ether by benzylammonium salts J. Polym. Sci., Part A: Polym. Chem. 1995 ;33: 505-512.

347 S. Nakano and T. Endo Thermal cationic curing with benzylammonium salts .2 Prog. Org. Coat. 1996 ;28: 143-148.

348 M. S. Kim, K. W. Lee, T. Endo and S. B. Lee Benzylpyrazinium salts as thermally latent initiators in the polymerization of glycidyl phenyl ether: Substituent effect on the initiator activity and mechanistic aspects Macromolecules 2004 ;37: 5830-5834.

349 Y. Yagci and I. Reetz Addition - Fragmentation type initiators for cationic polymerization Macromol. Symp. 1998 ;132: 153-164 .

350 Y. Yagci and I. Reetz Addition-fragmentation reactions in polymer chemistry React. Funct. Polym. 1999 ;42: 255264.

351 Y. Yagci, A. Onen and I. Reetz Structure and reactivity of addition fragmentation agents in photochemically and thermally induced cationic polymerization Macromol. Symp. 2001 ;174: 255-268.

352 A. Onen and Y. Yagci The effect of the heteroatom moiety of allylic salts on the addition fragmentation initiation of cationic polymerization Macromol. Chem. Phys. 2001 ;202: 1950-1954.

353 S. Denizligil, Y. Yagci and C. McArdle Photochemically and thermally-induced radical promoted cationic polymerization using an allylic sulfonium salt Polymer 1995 ;36: 3093-3098.

354 Y. Yagci and A. Onen An allylic pyridinium salt: Radical promoted latent thermal catalyst for cationic polymerization J. Polym. Sci., Part A: Polym. Chem. 1996 ;34: 3621-3624.

355 V. Bacak, I. Reetz, Y. Yagci and W. Schnabel Addition-fragmentation type initiation of cationic polymerization using allyloxy-pyridinium salts Polym. Int. $1998 ; 47:$ 345-350.

356 I. Reetz, V. Bacak and Y. Yagci Addition-fragmentation reactions for cationic polymerization using a novel allyloxypicolinium salt Polym. Int. 1997 ;43: 27-32.

357 L. Atmaca, A. Onen and Y. Yagci Allyloxy isoquinolinium salts as initiators for cationic polymerization Eur. Polym. J. $2001 ; 37: 677-682$.

358 A. Onen and Y. Yagci Initiation of cationic polymerization by using allyl anilinium salts in the presence of free radical initiators Macromolecules $2001 ; 34:$ 7608-7612.

359 M. K. Gupta and R. P. Singh Novel dibenzocycloheptenyl phosphonium salts as thermolatent initiator in cationic polymerization J. Appl. Polym. Sci. 2009 ;112: 3707-3713. 

xanthenyl phosphonium salts Macromol. Res. 2009 ;17: 221-226.

$361 \quad$ M. K. Gupta and R. P. Singh Novel allylic phosphonium salts in free radical accelerated cationic polymerization Polym. Bull. $\quad 2009 ; 62: 271-280$.

362 H. Y. Gu, K. T. Ren, O. Grinevich, J. H. Malpert and D. C. Neckers Characterization of iodonium salts differing in the anion J. Org. Chem. $2001 ; 66:$ 4161-4164.

363 K. T. Ren, J. H. Malpert, H. Y. Li, H. Y. Gu and D. C. Neckers Studies of weakly coordinating anions paired with iodonium cations Macromolecules 2002 ;35: 1632-1637.

364 M. Bednarek, P. Kubisa and S. Penczek Coexistence of activated monomer and active chain end mechanisms in cationic copolymerization of tetrahydrofuran with ethylene oxide Macromolecules 1999 ;32: 5257-5263.

365 M. Buhl and G. Wipff Hydronium ion complex of 18-crown-6: Where are the protons? A density functional study of static and dynamic properties J. Am. Chem. Soc. 2002 ;124: 4473-4480.

366 T. Vidil, F. Tournilhac and L. Leibler Control of cationic epoxy polymerization by supramolecular initiation Polym. Chem. 2013 ;4: 1323-1327.

367 A. Spath and B. Konig Molecular recognition of organic ammonium ions in solution using synthetic receptors Beilstein J. Org. Chem. 2010 ;6: 32/1-11.

368 M. Shamsipur and M.R. Ganjali Complex formation of some anilinium ion derivatives with 18-crown-6, 1,10diaza-18-crown-6 and cryptand C222 in acetonitrile, dimethylformamide and their 1:1 mixture J. Inclusion Phenom. Mol. Recognit. Chem. 1997 ;28: 315-323.

369 T. Akutagawa, H. Koshinaka, D. Sato, S. Takeda, S-I. Noro, H. Takahashi, R. Kumai, Y. Tokura and T. Nakamura Ferroelectricity and polarity control in solid-state flip-flop supramolecular rotators Nat. Mater. 2009 ;8: 342-347.

370 T. Akutagawa, D. Sato, H. Koshinaka, M. Aonuma, S-I. Noro, S. Takeda and T. Nakamura Solid-state molecular rotators of anilinium and adamantylammonium in [Ni(dmit)2]- salts with diverse magnetic properties Inorg. Chem. 2008 ; 47: 5951-5962.

371 J. Xu and M-M. Zhao 4-Chloro-anilinium tetra-fluoro-borate 18-crown-6 clathrate Acta Crystallogr. Sect. E: Struct. Rep. Online 2012 ;68: 0738/1-7.

372 J. V. Crivello and M. F. Aldersley Supramolecular Diaryliodonium Salt-Crown Ether Complexes as Cationic Photoinitiators J. Polym. Sci., Part A: Polym. Chem. 2013 ;51: 801-814.

373 T. Vidil and F. Tournilhac Supramolecular control of propagation in cationic polymerization of room temperature curable epoxy compositions Macromolecules 2013 ; 46: 9240-9248.

374 M. Morell, D. Foix, A. Lederer, X. Ramis, B. Voit and A. Serra Synthesis of a new multiarm star polymer based on hyperbranched poly(styrene) core and poly(epsilon-caprolactone) arms and its use as reactive modifier of epoxy thermosets J. Polym. Sci., Part A: Polym. Chem. 2011 ;49: 4639-4649.

375 M. Morell, X. Fernandez-Francos, J. Gombau, F. Ferrando, A. Lederer, X. Ramis, B. Voit and A. Serra Multiarm star poly(glycidol)-block-poly(styrene) as modifier of anionically cured diglycidylether of bisphenol A thermosetting coatings Prog. Org. Coat. 2012 ;73: 62-69.

376 M. Morell, X. Ramis, F. Ferrando and A. Serra New improved thermosets obtained from diglycidylether of bisphenol a and a multiarm star copolymer based on hyperbranched poly(glycidol) core and poly(methyl methacrylate) arms Macromol. Chem. Phys. 2012 ;213: 335-343. 

poly(ethyleneimine) core and poly(epsilon-caprolactone) arms as modifiers of diglycidylether of bisphenol A thermosets cured by 1-methylimidazole React. Funct. Polym. 2013 ;73: 431-441.

X. Fernandez-Francos, W. D. Cook, J. M. Salla, A. Serra and X. Ramis Crosslinking of mixtures of diglycidylether of bisphenol-A with 1,6-dioxaspiro 4.4 nonan-2,7-dione initiated by tertiary amines: III. Effect of hydroxyl groups on network formation Polym. Int. 2009 ;58: 1401-1410.

379 C. Acebo, A. Picardi, X. Fernandez-Francos, S. De la Flor, X. Ramis and A. Serra Effect of hydroxyl ended and end-capped multiarm star polymers on the curing process and mechanical characteristics of epoxy/anhydride thermosets Prog. Org. Coat. 2014 ;77: 1288-1298.

380 J. Libiszowski, R. Szymanski and S. Penczek On the cationic polymerization of oxirane with triphenylmethylium salts Makromol. Chem. Macromol. Chem. Phys. 1989 ;190: 1225-1232.

381 P. Kubisa and S. Penczek Cationic activated monomer polymerization of heterocyclic monomers Prog. Polym. Sci. 1999 ;24: 1409-1437.

382 M. Bednarek, P. Kubisa and S. Penczek Polymerization of propylene-oxide by activated monomer mechanismkinetics Makromol. Chem. Macromol. Chem. Phys. 1989 ;15: 49-60.

383 M. Bednarek, T. Biedron, P. Kubisa and S. Penczek Activated monomer polymerization of oxiranes microstructure of polymers vs kinetics and thermodynamics of propagation Makromol. Chem. Macromol. Symp. 1991 ;42-3: 475-487.

384 T. Biedron, K. Brzezinska, P. Kubisa and S. Penczek Macromonomers by activated polymerization of oxiranes. Synthesis and polymerization Polym. Int. 1995 ;36: 73-80.

385 Y. S. Li, M. S. Li and F. C. Chang Kinetics and curing mechanism of epoxy and boron trifluoride monoethyl amine complex system J. Polym. Sci., Part A: Polym. Chem. 1999 ;37: 3614-3624.

386 J. M. Morancho, A. Cadenato, X. Ramis, X. Fernandez-Francos and J. M. Salla Thermal curing and photocuring of an epoxy resin modified with a hyperbranched polymer Thermochim. Acta $2010 ; 510: 1-8$.

387 D. Foix, X. Ramis, M. Sangermano and A. Serra Synthesis of a new hyperbranched-linear-hyperbranched triblock copolymer and its use as a chemical modifier for the cationic photo and thermal curing of epoxy resins J. Polym. Sci., Part A: Polym. Chem. 2012 ;50: 1133-1142.

388 J. M. Morancho, A. Cadenato, X. Ramis, M. Morell, X. Fernandez-Francos, J. M. Salla and A. Serra Unexpected differences between thermal and photoinitiated cationic curing of a diglycidyl ether of bisphenol A modified with a multiarm star poly(styrene)-b-poly(epsilon-caprolactone) polymer Express. Polym. Lett. 2013 ;7: 565-576. J. Maria Morancho, A. Cadenato, X. Ramis, M. Morell, X. Fernandez-Francos, J. Maria Salla and A. Serra Thermal curing and photocuring of a DGEBA modified with multiarm star poly(glycidol)-b-poly(epsilon-caprolactone) polymers of different arm lengths J. Therm. Anal. Calorim. $2013 ; 114:$ 409-416.

390 X. Fernandez-Francos, J. Maria Salla, A. Cadenato, J. Maria Morancho, A. Serra, A. Mantecon and X. Ramis A new strategy for controlling shrinkage of DGEBA resins cured by cationic copolymerization with hydroxylterminated hyperbranched polymers and ytterbium triflate as an initiator J. Appl. Polym. Sci. 2009 ;111: 2822-2829.

391 D. Foix, M. Erber, B. Voit, A. Lederer, X. Ramis, A. Mantecon and A. Serra New hyperbranched polyester modified DGEBA thermosets with improved chemical reworkability Polym. Degrad. Stab. 2010 ;95: 445-452. D. Foix, X. Fernandez-Francos, J. M. Salla, A. Serra, J. M. Morancho and X. Ramis New thermosets obtained from bisphenol A diglycidyl ether and hydroxyl-ended hyperbranched polymers partially blocked with benzoyl and trimethylsilyl groups Polym. Int. 2011 ;60: 389-397. 

hyperbranched poly(glycidol) on the cationic curing and morphology of diglycidylether of bisphenol A thermosets React. Funct. Polym. 2011 ;71: 380-389.

394 A. Arnebold, K. Thiel, E. Kentzinger and A. Hartwig Morphological adjustment determines the properties of cationically polymerized epoxy resins RSC Adv. $2015 ; 5:$ 42482-42491.

395 A. Arnebold, O. Schorsch, J. Beckmann and A. Hartwig Concomitant cationic polymerization of a hybrid monomer and an epoxy resin React. Funct. Polym. 2013 ;73: 1625-1631.

396 H. Luetzen and A. Hartwig Dynamics in poly(epsilon-caprolactone) containing phase separated epoxy thermosets Macromol. Mater. Eng. 2013 ;298: 1275-1281.

397 H. Luetzen and A. Hartwig Highly cross-linked but tough: combination of contradicting properties in cationically polymerized epoxy-polyol adhesives J. Adhes. Sci. Technol. 2013 ;27: 2531-2541.

398 H. Luetzen, T. M. Gesing and A. Hartwig Nucleation as a new concept for morphology adjustment of crystalline thermosetting epoxy polymers React. Funct. Polym. 2013 ;73: 1038-1045.

399 H. Luetzen, P. Bitomsky, K. Rezwan and A. Hartwig Partially crystalline polyols lead to morphology changes and improved mechanical properties of cationically polymerized epoxy resins Eur. Polym. J. 2013 ;49: 167-176.

400 T. Biedron, R. Szymanski, P. Kubisa and S. Penczek Kinetics of polymerization by activated monomer mechanism Makromol. Chem. Macromol. Symp. 1990 ;32: 155-168.

401 B. J. Anderson Cationic cure kinetics of a polyoxometalate loaded epoxy nanocomposite J. Polym. Sci., Part A: Polym. Chem. 2012 ;50: 4507-4515.

402 J. V. Crivello, D. A. Conlon, D. R. Olson and K. K. Webb The effects of polyols as chain transfer agents and flexibilizers in photoinitiated cationic polymerization J. Radiat. Curing 1986 ;13: 3-9.

403 L. R. Gatechair and S. P. Pappas Cationic photoinitiation efficiency ACS Symp. Ser. 1983 ;212: 173-189.

404 S. P. Pappas, B. C. Pappas, L. R. Gatechair, J. H. Jilek and W. Schnabel Photoinitiation of cationic polymerization .4. Direct and sensitized photolysis of aryl iodonium and sulfonium salts Polym. Photochem. 1984 ;5: 1-22.

405 S. P. Pappas Photoinitiation of cationic and concurrent radical-cationic polymerization .5 Prog. Org. Coat. 1985 ;13: 35-64.

406 J. V. Crivello and R. A. Ortiz Benzyl alcohols as accelerators in the photoinitiated cationic polymerization of epoxide monomers J. Polym. Sci., Part A: Polym. Chem. 2002 ;40: 2298-2309.

407 R. A. Ortiz, D. P. Lopez, M. D. G. Cisneros, J. C. R. Valverde and J. V. Crivello A kinetic study of the acceleration effect of substituted benzyl alcohols on the cationic photopolymerization rate of epoxidized natural oils Polymer 2005 ;46: 1535-1541.

408 Y. J. Hua, F. M. Jiang and J. V. Crivello Photosensitized onium-salt-induced cationic polymerization with hydroxymethylated polynuclear aromatic hydrocarbons Chem. Mater. 2002 ;14: 2369-2377.

409 Z. Wang, X. Lin and W. Liu Synthesis of bis(2,3-epoxycyclohexyl) and its cationic photopolymerization in the presence of different diols Polym. Int. $2009 ; 58: 74-80$.

410 J. V. Crivello Structure-reactivity relationships of epoxide monomers in photoinitiated cationic polymerization in: J.

P. Fouassier and X. Allonas, editors. Basics and Applications of Photopolymerization Reaction Trivandrum:Research Signpost 2010 101-117.

411 H. Wang, R. Zhu, P. Yang and Y. Gu A study on the chain propagation of benzoxazine Polym. Chem. 2016 ;7: 860-866. 